\title{
Interventions to control children's screen use and their effect on sleep: a systematic review and meta-analysis
}

\author{
Katie Bianca Martin \\ Masters of Clinical Science \\ JBI \\ School of Public Health
}

Faculty of Health and Medical Sciences

The University of Adelaide

December 2020 


\section{Contents}

$\begin{array}{ll}\text { Abstract } & 4\end{array}$

$\begin{array}{ll}\text { Declaration } & 6\end{array}$

$\begin{array}{ll}\text { Acknowledgements } & 7\end{array}$

$\begin{array}{lr}\text { Chapter } 1 \text { Introduction } & 8\end{array}$

1.1 Screen use by children $\quad 9$

1.1.1 Screen time estimates in children 9

1.1.2 Television viewing 10

1.1.3 Mobile devices 11

1.1.4 Influence of demographics and family characteristics 11

1.2 Health effects associated with screen exposure 12

a. Obesity and metabolic health 13

b. Behaviour and mental health $\quad 14$

c. Academic performance 14

$\begin{array}{ll}\text { 1.3 Screen use and sleep } & 15\end{array}$

1.3.1 Mechanisms by which screen use may disrupt sleep $\quad 17$

1.3.2 Consequences of sleep insufficiency in children 18

1.3.3 Guidelines for screen use and sleep in children 19

a. Screen time recommendations 19

b. Sleep guidelines 20

1.3.4 Interventions to control screen use in children 21

1.4 Methodological basis for this review 21

1.4.1 Defining screen use 21

1.4.2 Methodology 22

1.4.3 Why this review is needed 24

Chapter 2 The systematic review protocol (Publication) 26

Chapter 3 The systematic review (Publication) 38

Chapter 4 Discussion and conclusions $\quad 72$

4.1 Influence of intervention parameters on outcomes 72

$\begin{array}{lll}\text { 4.1.1 Intervention 'focus' } & 73\end{array}$

4.1.2 Intervention content and specific strategies 74

a. Television monitoring devices 75

b. Removal of bedroom television 75

c. Restricting bedtime screen use $\quad 76$

4.1.3 Intervention duration and setting 76 
4.2.1 Participant age 77

4.2.2 Gender 77

4.3 Intervention effect and screen time and sleep guidelines $\quad 78$

4.4 Intervention effect and influence of uncontrolled variables $\quad 78$

$\begin{array}{lll}4.4 .1 & \text { Background screen exposure } & 79\end{array}$

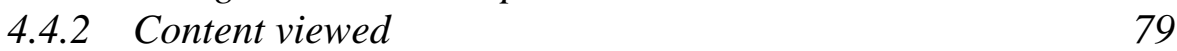

4.4.3 Parenting self-efficacy and parental modelling 80

$\begin{array}{ll}\text { 4.5 Sustainability and reporting of harms } & 81\end{array}$

$\begin{array}{ll}4.6 \\ \text { Limitations } & 82\end{array}$

4.6.1 Within-study limitations $\quad 82$

a. Defining and measuring screen use $\quad 82$

b. Sleep duration as an outcome 84

c. Measurement of sleep duration 85

d. Timing of data collection $\quad 86$

4.6.2 Limitations of the systematic review process 86

4.7 Implications for future research $\quad 87$

4.8 Implications for practice $\quad 89$

$\begin{array}{ll}4.9 \text { Conclusion } & 90\end{array}$

$\begin{array}{ll}\text { Appendices } & 92\end{array}$

$\begin{array}{ll}\text { I. Search strategy } & 92\end{array}$

$\begin{array}{lll}\text { II. Critical appraisal tools } & 94\end{array}$

a. Randomised controlled trials 94

b. Quasi-experimental studies 99

III. Data extraction form 100

$\begin{array}{ll}\text { IV. Statistical methods } & 102\end{array}$

a. Estimating the correlation coefficient 102

b. Variance for mean change 104

c. Standard deviation from the $95 \%$ confidence interval 105

$\begin{array}{ll}\text { V. Excluded studies } & 107\end{array}$

$\begin{array}{ll}\text { VI. Outcome measures utilised by included studies } & 111\end{array}$

$\begin{array}{ll}\text { VII. Statements of authorship } & 114\end{array}$

a. The systematic review protocol (publication) 114

b. The systematic review (publication) 115

$\begin{array}{lr}\text { Reference list } & 116\end{array}$ 
Prolonged viewing of screen-based media is associated with poor sleep in children. Previous systematic reviews have analysed the effectiveness of interventions aiming to limit children's screen use, however, none have evaluated their effect on sleep. The objective of the systematic review and meta-analysis presented in this thesis was to determine the effect of interventions that incorporate strategies to control children's screen use on improving screen use and sleep.

The databases PubMed, Embase, Eric, Scopus and PsycINFO were searched in October 2017 and updated in February 2019. Studies selected for inclusion used an experimental design to assess interventions to control screen use in children aged two to 14 years and reported outcomes for both screen use and sleep.

From 3,872 initial records, 11 studies (six randomised controlled trials [RCT], four clusterRCTs and one cluster, quasi-experimental) were eligible for inclusion. A total of 4,656 children aged two to 13 years were included. Studies generally inadequately controlled for potential sources of bias, particularly the use of subjective measures for screen-use and sleep. The included interventions focussed on education to control screen-use, delivered to the carer and/or child at school or home, either via face-to-face, online or posted hard-copy. Common behaviour modification strategies to control screen use included use of media budgeting, goal setting, provision on alternative activities, ensuring mealtimes and bedtimes are screen-free and 'screen-free' challenges. Interventions ranged from two weeks to 12 months duration. The mean reduction in screen time was 0.56 hours (33 minutes) per day (95\% CI $=0.92,0.20$; seven studies) and the mean sleep duration increased by 0.19 hours (11 minutes) per day (95\% CI $=0.05,0.33$; nine studies). Weekday bedtime advanced by 0.16 hours (10 minutes) and by 1.0 hour on the weekend. Subgroup analyses indicated that improvements in sleep duration and reduced screen time was greater with interventions of shorter duration (less than three months); that specifically targeted screen use or sleep or had direct participant contact.

In conclusion, small improvements in screen time and sleep duration can be achieved in children through interventions designed to control screen use. It is not possible to determine if a reduction in screen time directly improves sleep due to the limited number of studies, the presence of co-interventions, and issues with study methodological quality and 
heterogeneity. It is recommended that future research employ objective measures of screen use and sleep outcomes and attempt to measure or control factors known to influence these outcomes, such as background screen use and exposure to age-inappropriate or violent content. 


\section{Declaration}

I, Katie Bianca Martin, certify that this work contains no material that has been accepted for the award of any other degree or diploma in my name, in any university or other tertiary institution and, to the best of my knowledge and belief, contains no material previously published or written by another person, except where due reference has been made in the text. In addition, I certify that no part of this work will, in the future, be used in a submission in my name, for any other degree or diploma in any university or other tertiary institution without the prior approval of The University of Adelaide and, where applicable, any partner institution responsible for the joint award of this degree.

I acknowledge that copyright of published works contained within this thesis resides with the copyright holder(s) of those works.

I also give permission for the digital version of my thesis to be made available on the web, via the University's digital research repository, the Library Search, and also through web search engines, unless permission has been granted by the University to restrict access for a period of time.

I acknowledge the support I have received for my research through the provision of an Australian Government Research Training Program Scholarship.

Katie Bianca Martin

$20^{\text {th }}$ November 2020 


\section{Acknowledgements}

Completion of this master's thesis would not have been possible without the generous support and encouragement of key people, whom I would like to acknowledge.

For his encouragement, realism, and contagious enthusiasm for all things research, I would like to thank my principal supervisor, Associate Professor Edoardo Aromataris. Without his guidance, patience and support, this thesis would never have been completed, instead abandoned under a pile of research papers and half-filled data extraction forms.

Thank you to statistician Jana Bednarz who managed to convert each alien equation into a step-by-step easy-to-follow tutorial and checked my scanned hand-written sums.

I would like to thank my co-supervisor, Dr Kylie Porritt, particularly for her advice in the development of my review protocol and final review of this thesis.

The experience and knowledge of Maureen Bell was invaluable in developing the initial search strategy and fellow student Judith Lowe kindly performed the second appraisal of study quality.

Thanks to Ms Melanie Dankel, who undertook professional copyediting of this thesis in accordance with the Australian Standards for Editing Practice (specifically sections D and E) and Institute of Professional Editors thesis guidelines.

To my now four- and six-year-old children, if you ever read this, sorry for the grumpy mummy, let's attribute it to too much evening screen time in front of a computer. I hope one day you might appreciate your relatively screen-free childhood! 


\section{Chapter 1 Introduction}

Children today, particularly those in developed countries, have unprecedented access to electronic screen-based media. Screen-based devices are now easily transportable and onhand in situations and environments where previously there was limited access, such as during day-to-day movements outside the home. Hours spent viewing screen-based media mount quickly, and it is not unusual for teenagers to exceed six hours of screen time per day, ${ }^{1}$ with weekly screen time reportedly reaching 31 hours a week in primary school children. ${ }^{2}$ The portable nature of mobile screen devices has seen more screen use within the sleep environment, ${ }^{3}$ with an estimated one in six children in Australia aged two to four years able to access at least one item of screen-based electronic media in the bedroom, increasing to $51 \%$ of children aged five to 17 years. ${ }^{4}$ Similar trends are seen in other developed countries. ${ }^{5}$ More recently, an association has been found between screen use and poor sleep, particularly when viewed in the hour before bedtime. ${ }^{6}$ The detrimental effect on sleep is further exacerbated by violent, interactive or cognitively arousing content. ${ }^{7-9}$ In a systematic review of screen time and sleep in children, Hale and Guan ${ }^{6}$ report that of the 67 articles included, $90 \%$ found a significant adverse association between screen use and at least one sleep outcome. The American Academy of Pediatrics (AAP) ${ }^{9}$ recommends limiting screen use in children, and studies on the effectiveness of interventions targeting a reduction in children's screen time have been published. ${ }^{10-17}$ Previous systematic reviews have focused on how reducing screen time may impact body mass index (BMI); however, no systematic review to date has investigated how controlling screen use may affect sleep in children. In the absence of such a review, and with sleep outcomes measured in primary research targeting screen-time reduction, the objective of the systematic review presented in this thesis was to determine the effect of interventions that control screen use in children and how these may affect children's sleep.

In the first chapter of this thesis, a background to children's screen use and sleep is provided, followed by the methodological basis for this review. The published systematic review protocol ${ }^{18}$ is presented in chapter 2 . The systematic review, ${ }^{19}$ as published in the Journal of Sleep Research, is presented in chapter 3. The protocol details a full review project evaluating the control of screen use and other behavioural outcomes in addition to sleep, with only the sleep-related outcomes presented in this thesis. Information regarding the development of the search strategy is outlined in chapter 2 (section: search strategy) and 
chapter 3. The full, a priori search strategies employed across the range of databases are provided in Appendix I. Details regarding statistical methods can be found in chapter 3 (section: methods).

In chapter 4, the results and discussion as presented in the published review, are extended to allow further consideration of the results of the review, along with implications for future research. Due to the Thesis by Publication requirements, where chapter 2 and chapter 3 represent discrete, standalone manuscript submissions, some repetition of information, for instance introductory content in chapter 1, 2 and 3, is present and cannot be avoided. Due to individual journal requirements, chapter 2 is presented in US English and chapter 2 and chapter 3 each have a different referencing style and a unique reference list presented.

\subsection{Screen use by children}

The review presented in this thesis investigates interventions targeting the reduction or controlled use of electronic screen-based devices (referred to as 'screens') by children aged two to 14 years. The upper age limit was chosen primarily due to differing viewing habits and developmental needs of children versus adolescents, which may affect intervention content and delivery, and to align with the age at which children may finish primary school or middle school. The lower age limit was selected due to the many factors that may influence sleep in children under the age of two, such as sleeping arrangements and greater dependence on sleeping aids, ${ }^{20,21}$ the modification of which are likely to confound results of any screen-use intervention or present difficulties in ensuring compliance to an intervention.

\subsubsection{Screen time estimates in children}

Estimates of children's screen-hours have been reported in multiple large cross-sectional studies in Australia and internationally. ${ }^{1,2,5,22,23}$ According to the Australian Child Health Poll, ${ }^{2}$ which surveyed 3,797 participants from birth to 18 years, children viewed screens for over four hours per day on average. Nearly half of the teenage participants viewed screens for over six hours per day and one in five for more than 12 hours per day, averaging 43 hours in a week. Among primary school-aged children, weekly screen time averaged 31 hours, ${ }^{2}$ and an average of 25 hours per week was reported in children aged two to six years. ${ }^{2}$ Slightly lower total screen time estimates were reported in the Longitudinal Study of Australian Children (LSAC), ${ }^{22}$ with an average of 1.6 hours of screen time per day in children aged four to five years, increasing to 3.3 hours per day in children aged 12 to 13 years, with a rise in viewing times over the weekend period. Similar findings have been recorded in other 
developed countries. ${ }^{5}$ For example, in a study of over 2,500 children in the US, daily screen time averaged over four hours for children aged eight to 12 years, and over six hours in children aged 13 to 18 years. ${ }^{1}$ These estimates of daily screen time further increased with the inclusion of screen use for educational purposes, reaching a total of nearly six hours among the younger children and close to nine hours of daily screen time for the older children. ${ }^{1}$

While these estimates may reflect the screen-time habits of children from developed countries, excessive screen time in children has also been reported in countries with lower GDP or developing countries, though to a lesser extent. ${ }^{5}$ The widespread use of television was reported by Braithwaite and colleagues ${ }^{5}$ in a cross-sectional study of over 280,000 children and adolescents from 37 different countries. Among the participants surveyed, 79\% of children and $89 \%$ of adolescents reportedly viewed at least one hour of television per day. The proportion of adolescents reporting over three hours of television viewing per day ranged from $17 \%$ in China to $78 \%$ in Cote D'Ivoire, compared to $56 \%$ in the US, and in children, ranged from $8 \%$ in India to $36 \%$ in Nigeria. ${ }^{5}$ These figures represent television viewing hours only and not total screen time, including other screen devices, and may reflect the greater affordability and accessibility of television globally compared to other forms of screen-based media. ${ }^{5}$ For instance, it seems reasonable to expect more than $17 \%$ of adolescents in China to exceed three hours of daily screen time ${ }^{5}$ given mobile phone or tablet use has been found to account for $40 \%$ of total screen time in Chinese youths. ${ }^{24}$

\subsubsection{Television viewing}

Screen preferences and viewing habits differ depending on age. When considering younger children, despite the introduction of mobile devices, television viewing still accounts for the majority of screen time, with peak viewing occurring during the morning and midafternoon. ${ }^{22}$ Compared to earlier this century, an increase in daytime television viewing is evident, attributed to the increase in the availability of dedicated children's channels offering child-oriented content throughout the day. ${ }^{22}$ In contrast, peak television viewing for older children occurs between $7 \mathrm{pm}$ and $8 \mathrm{pm} .^{22}$ When considering an entire household, total television viewing hours increase as the number of televisions in the home increase. It is not uncommon for households to have more than one television; for instance, of the 10,000 participants from the $\mathrm{LSAC},{ }^{22} 42 \%$ had two televisions and $18 \%$ had at least three televisions in the home. The likelihood of children viewing less than one hour of television per day increased when only one television was present in the household. ${ }^{22}$ 
Total screen time increases with the presence of a television in the bedroom. ${ }^{22-25}$ The LSAC $^{22}$ study found that one in four children aged six to 11 years had a television in their bedroom, and among children aged 11 to 12 years, almost $50 \%$ had the capacity to watch television programs in their bedroom (this included television programs streamed to mobile devices). The children with bedroom television access were significantly more likely to watch more than two hours of television per day than children without access to television in the bedroom. ${ }^{22}$ Similar trends were observed by Wethington et al. ${ }^{23}$ in a sample of 29,000 US children, where the prevalence of excessive screen use doubled with the presence of a television in the bedroom; while Ye et al. ${ }^{24}$ found that children with a television in their bedroom spent three times as many hours playing videogames on Sundays compared to children without a bedroom television set. ${ }^{24}$

\subsubsection{Mobile device use}

Television may continue to be the most prevalent type of screen viewed among young children, however, preference for mobile screens increases with age. This is reflected in the amount of mobile phone ownership or personal mobile phone use among children. For example, of 6,000 UK adolescents, $75 \%$ of children aged 12 to 14 years had a personal mobile phone, ${ }^{26}$ compared to $94 \%$ in a sample of 1,093 Australian adolescents aged 13 to 18 years, ${ }^{22}$ while from a smaller sample of 651 pre-school children, $36 \%$ had a personal mobile screen device. ${ }^{2}$ Personal smartphone ownership was reported by $67 \%$ of children aged 13 to 18 years in a sample of over 2, 500 US children. ${ }^{1}$ Access to electronic games on mobile devices was observed to increase with age, from $56 \%$ of six- to seven-year-olds to $96 \%$ of 12- to 13 -year-olds. ${ }^{22}$ Gender differences in screen use and content become apparent among older children, with a preference in mobile phone use by adolescent girls for instant messaging, social media and music, and preference in adolescent boys for computer/internet, YouTube, interactive role-playing games ${ }^{1}$ and videogames/electronic games. ${ }^{1,22,24}$

\subsubsection{Influence of demographics and family characteristics}

Excessive screen time is consistently shown to be more prevalent in children of families belonging to particular sociodemographic groups. ${ }^{22}$ Lower parental educational attainment is shown to have a statistically significant association with increased screen time in children and increased (though not statistically significant) computer gaming hours. ${ }^{22}$ Children from low-income families are more likely to record excessive screen time, with adolescents viewing on average 2.45 hours more daily screen time than those from higher-income 
families. ${ }^{1}$ Migrant and Indigenous populations have been identified as being at greater risk of excessive screen time, after controlling for parental income and level of education, ${ }^{27-29}$ particularly if the parent had recently migrated. ${ }^{28,29}$

In exploring factors associated with screen use, it is essential to consider the capacity of the parent or caregiver in directly controlling screen use in young children and establishing healthy media habits in adolescents. Low parental self-efficacy, ${ }^{30}$ the presence of high 'household chaos', ${ }^{31}$ high parental screen use ${ }^{30,31}$ and families with fewer screen time 'rules' or limits ${ }^{22}$ are consistently shown to be risk factors for increased screen time. Similarly, greater screen exposure has been found among children of parents experiencing depression or stress. ${ }^{32}$

The attitude of the parent toward screen time and the potential perceived benefits of screen-use (e.g. enhances learning, brings enjoyment, useful parenting tool for occupying children) have been found to predict the screen time hours of their children. ${ }^{33}$ However, higher screen time in children was more strongly predicted by the presence of high parental time-pressure and maternal distress, even among parents who believed in the importance of restricting screen time. ${ }^{33}$ It has been proposed that the cognitive dissonance experienced by such parents may prompt parents to attempt to achieve consistency between attitudes and behaviours, and alleviate associated feelings of guilt, by adjusting their attitudes toward screen time to match their child's level of screen time. ${ }^{33}$

\subsection{Health effects associated with screen exposure}

The majority of studies investigating the health effects of screen exposure are observational cross-sectional or cohort studies. ${ }^{34}$ While most of these studies present consistent results regarding factors associated with excessive screen time, in the absence of large studies utilising an experimental design, drawing conclusions and determining causality is difficult. There is evidence, based on these studies, to suggest that excessive screen time is likely to be associated with multiple detrimental effects to health and well-being. ${ }^{32}$ One difficulty in attempting to determine the true extent of the health effects associated with excessive screen time in children is obtaining a large enough sample of children with zero screen exposure that still has a representative or comparable mix in other participant characteristics at baseline, such as socioeconomic status, and educational attainment. For instance, in the LSAC, less than $1 \%$ of approximately 10,000 participants did not have a television in the home. ${ }^{22}$ While experimental studies aiming to reduce screen time and investigating how this may affect health outcomes exist, most of these studies target multiple lifestyle behaviours 
for change, presenting another difficulty in establishing the strength of association between health effects and screen exposure. ${ }^{35}$ For example, interventions aiming to improve sleep may address screen time, diet (reducing caffeine), the establishment of regular morning wake time, good sleep hygiene, and physical activity, all of which can affect the outcome of interest, making it difficult to determine if any improvement in sleep was a result of reducing screen time or due to a culmination of lifestyle changes.

Since the introduction of electronic media via the television, research has been conducted on the potential health effects such exposure has on children. Early research sought to determine if exposure to violent content was associated with aggression in children. ${ }^{36}$ Later, when concerns were raised in relation to the increasing levels of obesity in children, research investigated correlations between television viewing hours, both as a measure of sedentary activity and as an outcome itself, and body weight. ${ }^{5}$ Today, the interest in the health effects of electronic media on children extends beyond television to include the array of mobile devices, some of which are briefly discussed here. Although screen time hours are on average greater among families of lower socioeconomic status and lower parental educational attainment, the health effects of excessive screen time are present after controlling for these factors.

\section{a. Obesity and metabolic health}

Ongoing excessive screen use is associated with overweight in children and significant doseresponse relationships have been found between increased television viewing and increased obesity. ${ }^{5,37,38}$ For instance, in a meta-analysis by Zhang et al. ${ }^{37}$ the risk of childhood obesity was found to increase by $13 \%$ for each hour per day increment in television viewing. As research investigating the effects of excessive screen use on metabolic health indicators besides obesity in children is limited, it is useful to consider how screen time correlates with metabolic health in adults, particularly as the screen-use habits established in childhood often continue into adolescence and adulthood. ${ }^{4,15,22}$ Similar trends to that reported by Zhang et al. ${ }^{37}$ were drawn from a meta-analysis of 58 studies and over 1,000,000 adult participants by Guo and colleagues, ${ }^{38}$ in which the risk of being overweight increased by $53 \%$ when viewing television for three hours per day Guo et al. ${ }^{38}$ also reported a linear relationship between television viewing and risk of hypertension, which increased by $6 \%$, and type 2 diabetes, which increased by $8 \%$ for each corresponding additional hour of television viewing per day. ${ }^{38}$ The mechanism underlying these results is not yet understood but is 
potentially attributable to exposure to fast food advertising, consuming food while watching television, and screen time being a primarily sedentary activity. ${ }^{39}$

\section{b. Behaviour and mental health}

Excessive screen use is associated with increased anxiety, depression, aggression ${ }^{40}$ and antisocial behaviour, ${ }^{34}$ after controlling for demographic factors. In a population-based, crosssectional survey of over 5,000 US children, exposure to media violence was strongly associated with physical aggression in children, after adjusting for socioeconomic factors, family and community violence, and child mental health. ${ }^{40}$ The association between screen time and adverse well-being and behavioural outcomes may be due to exposure to ageinappropriate or violent content, tragic events, ${ }^{41}$ advertising and commercials or cyberbullying. As many as one in five parents have reported that their children had experienced on-line bullying. ${ }^{2}$ In a cohort study of over 7,000 Australian children aged six and 10 years, screen time was found to be associated with emotional problems in young children, and hyperactivity, poor prosocial behaviour and conduct problems in older children. ${ }^{42}$ Important effect moderators were the level of parental education, income, and neighbourhood socioeconomic status. Similarly, in a sample of over 40,000 US children aged two to 17 years, after an hour of screen time per day, each additional hour of electronic media viewed was associated with lower emotional well-being, lower capacity for maintaining attention, and reduced social skills in children and adolescents. ${ }^{43}$ Such findings were supported in a systematic review of the effects of television viewing on children's cognition, which demonstrated an association between excessive screen time and poorer concentration and attention. ${ }^{44}$

Differentiating between recreational screen use and use of screen-based media for educational or work purposes, such as completing homework, has been highlighted as an important consideration in screen time analyses, particularly as recreational screen time has been found to be more strongly associated with poorer mental health than non-recreational screen time. ${ }^{45}$

\section{c. Academic performance}

There is evidence to suggest that excessive screen time is associated with reduced academic achievement. ${ }^{46}$ This was apparent in a cross-sectional survey of 934 school-aged Australian children, where greater academic performance was seen in children with lower screen time hours. ${ }^{46}$ In contrast, Adelantado-Renau et al. ${ }^{47}$ systematically reviewed 58 cross-sectional 
studies with a total of 480,000 children aged four to 18 years, finding overall screen time was not significantly associated with poor academic performance; however, when analysed according to screen type, television viewing and videogaming were shown to be negatively associated with academic outcomes compared to other screen types. ${ }^{47}$

\subsection{Screen use and sleep}

Screen use at bedtime is associated with multiple adverse effects on sleep, such as reduced sleep duration, increased sleep onset/latency, increased night wakings, night terrors, and daytime drowsiness. The extent to which sleep may be affected appears to depend on the type of screen, the content viewed and the time of viewing. Bedroom access to screen-based media appears to further amplify the detrimental effects on sleep. ${ }^{6,23,48-50}$ For example, a survey of 2,334 $5^{\text {th }}$-grade children by Dube et $a l .{ }^{48}$ revealed shorter sleep duration among children with bedroom screen access. Reductions in sleep duration ranged from seven minutes with frequent access to a television at bedtime, to 10 minutes for access and frequent use of a mobile phone compared to no or rare access to screens at bedtime. ${ }^{48}$ Slightly greater differences in sleep duration between children with and without a bedroom television were found by Michaels et al. ${ }^{50}$ in a sample of over 16,000 children aged two to nine years, with sleep duration averaging nine hours and 43 minutes with a bedroom television set, compared to 10 hours and 15 minutes without. ${ }^{50}$

Bedroom screen access has been shown to affect other sleep outcomes in addition to sleep duration. For instance, a $43 \%$ reduction in good sleep quality was observed in children with bedroom screen access compared to children with no media access and who read a book before bed. ${ }^{48}$ Sleep efficiency was also reduced with access to and frequent use of screens at bedtime, ${ }^{48}$ along with slightly larger delays in bedtime, with bedtime delayed by 20 minutes if television was viewed at bedtime and increased sleep onset latency has been consistently reported. ${ }^{6}$

The incidence of general sleep problems in children has been shown to increase with evening screen use. A systematic review of 20 cross-sectional studies with 125,000 children aged six to 19 years reported a consistently strong association between portable screen use at bedtime and inadequate sleep duration and poor sleep quality. ${ }^{49}$ The negative effects of evening screen use was further demonstrated in the baseline data reported by Garrison and Christakis, ${ }^{51}$ where for each additional hour of evening screen use, an increase in sleep problems was observed. However, not all children using screens at bedtime may experience sleep problems; for instance in one study, of the children that were reported to use a screen 
device at bedtime, one in four experienced sleep problems. ${ }^{2}$ Such inconsistency may be indicative of the differences in content viewed and highlights the need for more detail in reporting screen use and screen content. The influence of the type of content viewed was analysed in a review by Hale and Guan, ${ }^{6}$ with greater delays in bedtime and total sleep time reduced by 51 minutes in adolescents using the internet for social reasons at bedtime compared to adolescents not using the internet at bedtime; and total sleep time reduced by 45 minutes in adolescents with access to a mobile phone at bedtime. ${ }^{6}$ When considering videogaming at bedtime, greater reductions in sleep duration are also observed. ${ }^{6,52}$ Children not receiving the recommended amount of sleep for their age are more likely to have internet access in the bedroom or spend more time on the internet. ${ }^{22}$

The greater reductions in sleep duration with mobile device use and interactive media compared with television reported by Dube et al. ${ }^{48}$ was similarly supported by Mireku et $a l .,{ }^{26}$ finding that mobile phone use at bedtime was associated with poor sleep outcomes (insufficient sleep duration and later sleep midpoint) and reduced health-related quality of life. Interestingly the association was further strengthened when the mobile phone was used in a dark room compared to a lit room. ${ }^{26}$ The results from these studies indicate that mobile device use and interactive content appear to have greater detrimental effects on sleep than viewing content on a television. Sleep duration has been found to be further reduced with access to multiple screen devices in the bedroom. ${ }^{6}$

The effects of screen use on sleep outcomes may not be limited to screen exposure immediately prior to bedtime. Dube et $a l^{48}$ observed that total daily screen use was associated with poorer sleep quality. Even daytime use of portable devices compared to daytime television may reduce sleep duration. ${ }^{53}$ For instance, of the 1,117 children aged one to two years in a cross-sectional study by Chindamo and colleagues,${ }^{53}$ daily smartphone or tablet use increased the odds of longer sleep onset latency and reduced total sleep time, irrespective of watching television and the child's temperament. ${ }^{53}$

Total daily screen time and its effect on sleep duration and disturbances was examined in a cross-sectional sample of 613 American children aged three to 17 years. ${ }^{54}$ Of the sample, $16 \%$ of children aged three to seven recorded less than two hours of screen time per day, compared to none of 13 to 17 year olds; $37 \%$ of three to seven year olds viewed screens for two to four hours per day, with similar hours recorded for children aged eight to 12 years; while $6.7 \%$ of children aged three to seven years viewed screens for over 10 hours per day, increasing to $9 \%$ of eight to 12 year olds and up to $27 \%$ of 13 to 17 year olds. The rate of sleep disturbances increased significantly in younger children after four to six hours of daily 
screen time, and after eight to 10 hours of screen time in older children and adolescents. ${ }^{54} \mathrm{~A}$ steady and statistically significant decrease in sleep duration in children aged three to 12 years was observed as screen time hours increased, however sleep duration in adolescents appeared to be unaffected by daily screen time hours, even at over 10 hours per day. ${ }^{54}$ This was supported by Rapoport and colleagues ${ }^{55}$ in the US National Survey of Children's Health ( $\mathrm{HSCH})$ of 12,642 children aged one to five years. Their findings showed that for each additional hour a child spent viewing electronic media devices in the daytime, including television, there was a greater likelihood of having inconsistent bedtime and inadequate sleep duration for their age. The possibility that excessive daily screen time hours in adolescents may not alter sleep duration highlights the importance of evaluating other sleep and screenuse outcomes to determine how screen use may affect sleep.

\subsubsection{Mechanisms by which screen use may disrupt sleep}

Mechanisms by which screen use influences sleep have been proposed, most of which pertain to evening screen use. Viewing screens and prioritising this activity over sleep may displace sleep time and postpone bedtimes ${ }^{56}$ Exposure to light emitted by screens at bedtime is thought to interrupt the natural circadian rhythm and the release of melatonin, a hormone required for sleep. ${ }^{57}$ In studies measuring the level of melatonin in the blood or saliva from healthy adults randomised to either exposure to bright light at bedtime or dull/dark room, ${ }^{58}$ bright light was found to significantly delay the rhythm of melatonin secretion by nearly two hours. ${ }^{58}$ Similarly, a 1.5 hour delay in the melatonin cycle, increased sleep latency and decreased subjective bedtime sleepiness was observed in adults randomised to read a book via an E-reader compared to those randomised to read the same book in printed form. ${ }^{59}$

To improve overall sleep, increasing daytime light exposure, preferably natural light, is frequently recommended. This was demonstrated by Rångtell et al. ${ }^{60}$ finding that constant prolonged exposure to bright light for 6.5 hours immediately prior to two hours of E-reader at bedtime, mitigated the effect that bright light at bedtime had on the timing of melatonin secretion/circadian rhythm. The results show the importance of daytime light exposure, however, acceptability or ease of implementation in practice was not discussed. ${ }^{60}$ The use of blue-light filters may also reduce the negative effect of exposure to light-emitting devices at bedtime. $^{61}$

Another important mechanism to consider is screen content. More interactive content, such as videogaming and social media, may heighten arousal levels ${ }^{8}$ and lengthen sleep latency. Exposure to violent content may affect sleep, as shown by Paavonen $e t a l .^{7}$ in a 
survey of 361 families of children aged five to six years, where viewing aggressive or adultdirected content, such as news, current affairs or police documentaries, either viewed passively/in the background, or purposely viewed by the child, were associated with greater sleep problems in the child. ${ }^{7}$ The same study found no correlation between daytime viewing of age-appropriate content and sleep problems in children. ${ }^{7}$ Similar results were reported by Garrison and Christakis, ${ }^{51,62}$ where an association with daytime viewing of violent media, including animated violence, live-action or realistic depictions of violence, and sleep problems in children was found, but the same effect was not observed for daytime viewing of non-violent content. In addition, exposure to age-inappropriate content or traumatic and tragic events, such as those depicted in mass media, may increase anxiety, ${ }^{41}$ which may in turn affect sleep quality. In terms of the acute physiological effects of exposure to violent screen content, a randomised controlled trial (RCT) by Invarsson et al. ${ }^{8}$ on violent versus non-violent videogaming content viewed by adolescents in the evening found increased heart rate and greater bedtime stress levels in the group with high-violence exposure. ${ }^{8}$ It is concerning, however, that these effects were less pronounced among participants with previous exposure to violent content, highlighting the importance of considering levels of pre-exposure in ensuring treatment group similarity at baseline. ${ }^{8}$ This suggests that children with high levels of exposure to violent media may have highly specific intervention needs.

Daytime screen viewing may also affect sleep by being a primarily sedentary activity. Excessive screen time may replace more active leisure pursuits and subsequently lower energy expenditure throughout the day, ${ }^{5}$ which is important for healthy sleep.

\subsubsection{Consequences of sleep insufficiency in children}

The importance of sleep for the healthy physiological and psychological development of children is well-recognised. ${ }^{63-65}$ Sleep insufficiency is associated with increased calorie intake, obesity, sedentary behaviour and more screen time, according to the findings of a systematic review of 69 studies with a total of 148,524 participants aged from birth to four years by Chaput et al. ${ }^{65}$ In addition to this, inadequate sleep tended to be associated with poorer emotional regulation and more injuries, ${ }^{65}$ reduced well-being and higher likelihood of depression and anxiety. ${ }^{22}$ Higher levels of daytime sleepiness, inattention, poorer academic achievement and behavioural difficulties in children with shorter sleep duration has been observed. ${ }^{22}$ It is important to note how many of the above outcomes are also those frequently found to be associated with excessive levels of screen time. 
Sleep insufficiency has been found to be significantly associated with a range of negative outcomes, including binge drinking, obesity, fighting, and contemplated and attempted suicide. ${ }^{66}$ Sleep insufficiency and its association with such risk-taking behaviour was explored by Meldrum and Restivo, ${ }^{66}$ in a survey of over 15,000 US adolescents aged 14 to 18 years. Of the study participants, only $31 \%$ met the recommended eight hours of sleep per night, $30 \%$ achieved seven hours per night, $22 \%$ achieved six hours and $11 \%$ achieved five hours, with the remaining $7 \%$ getting less than five hours sleep per night. The odds of engaging in one or more of the risk-taking behaviours mentioned increased with each additional hour of sleep curtailment, with eight hours of sleep being the referent. However, Meldrum and Restivo ${ }^{66}$ highlight that the odds of engaging in risk-taking behaviours increased substantially in participants achieving less than five hours of sleep per night and suggest mild to moderate sleep restriction may not be harmful. ${ }^{66}$ Meldrum and Restivo ${ }^{66}$ propose the link between sleep curtailment and risk-taking behaviour to be cognitive impairment, and recommend examining the odds of health problems and behavioural concerns across the continuum of sleep sufficiency and chronic sleep deprivation, particularly in younger subjects. Such findings highlight the importance of interventions to improve sleep in children and adolescents to optimise health and well-being outcomes.

Regular, good sleep is considered crucial for metabolic functioning. ${ }^{67}$ Mechanisms by which chronic sleep insufficiency may cause obesity and type 2 diabetes mellitus in adults have been proposed, ${ }^{67}$ with chronic short, mis-timed or disturbed sleep potentially altering metabolism via increasing stress levels, altering insulin sensitivity, desynchronising molecular clocks, culminating in reduced energy expenditure and increased food salience.

\subsubsection{Guidelines for screen time and sleep in children}

\section{a. Screen time recommendations}

In an effort to mitigate the potential risks associated with excessive screen use, the American Academy of Pediatrics (AAP) provide guidance on restricting screen time and ensuring appropriate content. ${ }^{68}$ Currently, according to the AAP, no screen time is recommended in infants under 18 months, at which age interactive video-chat and high-quality programs may be introduced. ${ }^{68}$ In children aged two to five years, less than one hour per day is recommended. ${ }^{68}$ However, for children over five years of age, no screen time limit is provided, and parents are encouraged to set screen time limits at their own discretion, ultimately ensuring that the child's screen use does not impact on their ability to engage in other required activities. ${ }^{68}$ Previously, the AAP recommended no screen time under two 
years of age and a limit of two hours per day was applied to all children over five years of age ${ }^{69}$ Many studies have used the two-hour limit to define acceptable or excessive levels of screen time, with hours of viewing beyond this limit considered excessive. The limit had been criticised as being unachievable given the amount of screen use at school and home ${ }^{70}$ and in 2016 was changed to the current guidelines. It is not surprising that approximately $63 \%$ of children aged two or less and $72 \%$ of children aged two to four years, viewed screens for more than the recommended one hour per day, and $85 \%$ of teenagers viewed screens for more than the recommended two hours per day. ${ }^{2}$ In 2019 the World Health Organization (WHO) released its first ever guidelines for screen use in children under five years of age, differing slightly from that set by the AAP, recommending no screen time whatsoever for children under two years, and matching the AAP with less than one hour per day for children aged two to five years. ${ }^{71}$

\section{b. Sleep guidelines for children}

Guidelines for children's sleep requirements recommend that children aged three to five years obtain between 11 and 13 hours of sleep per night; that children aged six to 13 years receive nine and 11 hours per night and that teenagers aged up to 17 years obtain between eight and 10 hours. $^{72}$ It is estimated that sleep insufficiency in children occurs in approximately $36 \%$ of children aged three to five years old, and $20 \%$ of children aged five to 12 years. ${ }^{73}$ The prevalence of sleep insufficiency appears to increase with age. For instance, of the participants in the LSAC, ${ }^{22}$ one in four children aged 12 to 13 years were not meeting recommended minimum sleep hours on a school night, increasing to $50 \%$ in older adolescents. ${ }^{22}$

Several practices and routines that include addressing screen use are recommended to optimise sleep in children, such as modifying the sleep environment and reducing stimulus levels at bedtime. ${ }^{72}$ These form part of good sleep hygiene, which refers to physical changes to the sleep environment and behavioural changes to promote sleep. ${ }^{74}$ Recommendations include keeping the bedroom screen-free (TV, computer, mobile devices) and avoiding viewing screens in the hour prior to bedtime, particularly interactive media. ${ }^{72}$ Establishing rules for appropriate media use in the family is important, as it has been found that adolescents from families with rules regarding the use of screen-based media at bedtime report an earlier bedtime than families with no restriction on media. ${ }^{75}$ 


\subsubsection{Interventions to control screen use in children}

The effectiveness of strategies specifically targeting screen-time reduction has been well researched. ${ }^{10-17}$ Strategies employed for reducing screen time in children often involved a combination of environmental changes, behavioural approaches and education. ${ }^{10-14}$ Environmental strategies involved removing the television or screen device from the bedroom, preventing screen access at mealtimes or utilising a television control device. ${ }^{11,13}$ Frequently used behavioural interventions include one or a combination of media budgeting; goal setting, ${ }^{11}$ 'television turn off week', ${ }^{76}$ providing alternative activities, rewards or incentives; counselling, ${ }^{17}$ or contingent feedback systems, such as screen time being contingent on time spent engaging in physical activity. ${ }^{11}$ Educational content typically addressed health promotion in the adverse health effects of excessive screen use, appropriate content for children, co-viewing, ${ }^{77}$ and skills in media literacy and being critical of media content. ${ }^{77-79}$

The above strategies are often implemented within programs provided to children and or their families as either one among many behaviours targeted for change or programs specifically focused on the outcome of interest, ${ }^{13}$ such as reducing or controlling screen use or improving sleep. They are typically carried out within the home or school environment. ${ }^{11,13}$ Most evaluate interventions primarily aimed at reducing childhood obesity and improving factors associated with obesity, such as nutrition, screen time, physical activity and sleep. To date, few studies evaluate interventions that solely address screen use, including screen time, content or restricting evening screen use, and fewer again include the outcome of sleep.

\subsection{Methodological basis for the review}

\subsubsection{Defining screen use}

Historically, studies have been primarily focused on 'screen time' and its association with obesity. In focusing only on the time spent viewing a screen, other important factors related to screen use are ignored, such as the content viewed or the time at which the content is viewed. While health promotion programs may focus on reducing sedentary behaviour via limiting screen time, other programs such as media literacy programs, behaviour management programs, or programs for improving sleep hygiene, may be concerned with other aspects of electronic media use, hence the term 'screen use'. For the purposes of this review, screen time refers to the total time spent viewing any electronic media on any screen- 
based device. This includes television, computers, and the numerous mobile screen devices. The term 'screen time' appears readily accepted and frequently used in research papers, though it is occasionally also referred to as 'media use', 'screen based electronic media', 'electronic entertainment and communication devices' or 'technology-use'. ${ }^{48,75}$ Unless specifically measured and indicated, total screen time generally does not include background screen exposure. For the purposes of this review, the term 'screen use' is used to refer not only to screen time but more generally, including the content viewed, such as videogaming or social media, the internet, as well as when, where and how it is used.

\subsubsection{Methodology}

The goal of a systematic review is to summarise the results of all available evidence in an unbiased, comprehensive, and transparent way. ${ }^{80}$ The demand, and indeed necessity, for the systematic review stems from the field of evidence-based health care, which recognises the importance of ensuring that clinical practice and healthcare systems are efficacious both clinically and economically and that they are supported by the best available evidence, ultimately optimising health care outcomes. ${ }^{80}$ The importance of evaluating the effectiveness of health care practices gained momentum during the $1990 \mathrm{~s}^{80}$ and continues to evolve in response to the challenges and requirements of ever-changing healthcare systems and rapidly developing treatment approaches. Despite primary research being generated, there is often low or slow evidence uptake in practice, in part due to the challenge of keeping up to date with the immense number of studies published daily, ${ }^{80}$ suggesting the need for greater support for the translation of research findings into practice.

The systematic review is considered the highest level of evidence and is frequently sought to inform, support and update decision-making in health care practice, and guide the direction of future research. ${ }^{80}$ Methods have been generated to eliminate potential sources of bias in the review process and create greater transparency, such as those recommended by JBI, which have been employed in the current review. ${ }^{80}$ The JBI systematic review process provides key steps in review completion, including the development of a protocol, which details the review question inclusion and exclusion criteria, the search strategy, the outcomes of interest and details of how they will be synthesised. ${ }^{80}$ The systematic review must be conducted according to the registered protocol, with any deviation from the protocol and associated reasoning stated within the review. Eligible studies are critically appraised by more than one person using pre-specified critical appraisal tools. The use of such explicit 
and pre-determined methods allows for greater transparency ${ }^{80}$ to the extent that another party could perform the same systematic review and achieve the same results.

There are several types of systematic review, selection of which is dependent on the nature of the review question. JBI offers extensive guidelines for the completion of qualitative and quantitative reviews (effectiveness; prevalence and incidence; aetiology and risk; diagnostic test accuracy; measurement accuracy), reviews of text and opinion, economic reviews, umbrella and scoping reviews, and mixed method reviews. ${ }^{80}$ As the question of the review presented in this thesis sought to determine the effect of an intervention, a quantitative systematic review of effectiveness was considered an appropriate method of review. This type of systematic review is designed to determine the degree to which the intervention of interest achieves the desired effect, and provide details of effect size, direction and, depending on the study designs involved, potential causality. ${ }^{80}$ Studies included in a review of effectiveness may be experimental, quasi-experimental or observational. ${ }^{80}$

In the current systematic review, inclusion was restricted to experimental and quasiexperimental studies, where a group of participants either receive an intervention or act as a control, with outcomes of interest for participants from both the treatment and control groups measured prior to group allocation and at intervention completion. ${ }^{80}$ Allocation to intervention or control group can occur by using an authentically random process, such as an RCT, a systematic or pseudo-random method (pseudo-RCT) or non-random allocation (quasi-experimental). RCTs are considered the most desirable method as they control for bias that may occur in treatment allocation by ensuring participants are randomly allocated to either an intervention or control group. An RCT may specify differing organisation aside from the typical parallel design, such as cross-over or cluster designs. However, the degree to which an RCT upholds the integrity of this method needs to be determined via critical appraisal. ${ }^{80}$ Factors to consider are the method of randomisation; allocation concealment; blinding of participants, trialists and outcome assessors; similarity between treatment groups at baseline; attention-matched intervention and control; that drop-outs are accounted for; and appropriate statistical methods are used, such as using intention to treat analysis. ${ }^{80}$ If a pseudo-random allocation is used, justification for the chosen method should be provided, and if cluster randomisation is used, details should be provided on how this was accounted for in analysis.

The process of combining and synthesising the results of multiple homogenous studies provides greater external validity and generalisation. When included studies are sufficiently 
homogeneous both clinically and methodologically, statistical pair wise meta-analysis can be performed. ${ }^{80}$ The benefits of meta-analysis include improved statistical power to determine treatment effect, provide a summary estimate and explore treatment effect among certain sub-groups or for different intervention variables. As the results of the meta-analysis are dependent on decisions regarding the application of certain statistical processes and assumptions, sensitivity analysis can be completed to test the influence of such decisions on the result. Sensitivity analysis can be performed by the sequential removal of each study; removal of outliers; application of different values in the analysis, such as correlation coefficient range; and observation of how this may alter the estimate and other relevant values. ${ }^{80}$ One appealing outcome of a meta-analysis is the generation of a forest plot. The forest plot provides a visual representation of the effect estimate size and direction for all included studies and any subgroup analysis, along with the summary estimate. This aids the reader in interpreting the results, however, the reader is advised to consider the results in relation to other important details, such as size of standard deviation and amount of statistical heterogeneity, represented by the width of the 'diamond' and $\mathrm{Tau}^{2}, \mathrm{Chi}^{2}$ and $I^{2}$ values. For results that are unable to be included in meta-analysis, results can be interpreted and synthesised in narrative form. With numerous methods of measuring screen time and sleep outcomes utilised in primary research, it was probable that some of the results of the present review would be able to be pooled in meta-analysis, and the remainder presented in narrative form.

\subsubsection{Why this review is needed}

Several systematic reviews with meta-analysis have investigated the effectiveness of interventions to reduce screen time in children, either with screen time as the single outcome of interest ${ }^{15}$ or considering how such interventions affect other outcomes, most frequently BMI. ${ }^{10-17}$ Other relevant systematic reviews attempt to determine the effectiveness of interventions aiming to reduce sedentary behaviour, the primary outcome measure of which is frequently screen time. ${ }^{12,76,81,82}$ The majority of included interventions targeted multiple behaviours for change, with screen-time reduction one of many lifestyle behaviours addressed, such as healthy diet, reducing sugary beverage intake, reducing sedentary behaviour or increasing physical activity, and improving sleep. Most interventions of this nature were effective in reducing screen time, achieving reductions ranging from $0.25^{10}$ to 0.44 hours per day. ${ }^{13}$ The intervention effect was often strengthened with subgroup analysis for age, higher proportion of content focusing on screen-time reduction, and involvement of family. In relation to participant age, reviews have shown inconsistent findings, with 
intervention effect strengthened in participants under the age of six in a review by Wahi et $a l .{ }^{16}$ (otherwise null findings are evident in participants aged three to 12 years), and strengthened in children aged over five years in a review by Maniccia et al. ${ }^{11}$

A review by Ramsey Buchanan et al. ${ }^{13}$ recommended future studies and reviews evaluate a broader range of outcomes that may be affected by modifying screen time, such as sleep. Literature addressing screen use and sleep is limited, and the few existing reviews and primary research investigating sleep interventions in children do not list screen use as an outcome, rather include controlling screen use within the intervention content, often within 'sleep hygiene practices' ${ }^{20,83}$ To date, no systematic review has investigated how controlling screen use may affect sleep outcomes in children. As research in the field of screen-use interventions and children's sleep is only emerging, with limited published studies available, studies that addressed screen-use behaviour within the intervention, such as broad lifestyle interventions with the primary aim of reducing obesity, that also included sleep-related outcomes, were considered appropriate for inclusion. Therefore, the aim of the current review was to determine the effect of interventions that include strategies to control screen use, either as a standalone intervention or within a multi-component lifestyle intervention, on screen-use and sleep outcomes in children. 


\section{Chapter 2 Systematic review protocol}

The following chapter contains the systematic review protocol ${ }^{18}$ as published in the JBI Database of Systematic Reviews and Implementation Reports, reproduced here with permission. The only modification to content was the removal of the $\operatorname{protocol}^{18}$ search strategy appendix, replaced with reference to Appendix I, which is presented in this thesis and details the full search strategy. 


\title{
Effectiveness of interventions to control screen use and children's sleep, cognitive and behavioral and outcomes: a systematic review protocol
}

\author{
Katie Martin $\cdot$ Kylie Porritt $\cdot$ Edoardo Aromataris \\ Joanna Briggs Institute, Faculty of Health and Medical Sciences, University of Adelaide, South Australia \\ Correspondence: Katie Martin, katie_m@y7mail.com \\ Co-author EA is the Editor-In-Chief of the JBI Database of Systematic Reviews and \\ Implementation Reports and was not involved in the editorial processing of the manuscript.
}

\section{Review questions}

i. What is the effect of intervention programs which include strategies to control screen use (total time, timing and/or content) on:

- children's sleep?

- children's behavior, including aggression, hyperactivity or prosocial behavior and wellbeing (depression and self-esteem)?

- children's selected cognitive skills: concentration, attention, executive function, engagement in play, school performance (e.g. following instruction, completing set activities, and academic achievement)?

ii. With the data available, what intervention program design characteristics (structure, setting, delivery, duration) are effective for screen time type (television, computer, or hand held devices) and participant age (toddler, child or young adolescent) or gender?

Keywords Childhood development; electronic media; intervention; mobile devices; screen time JBI Database System Rev Implement Rep 2018; 16(6):1338-1345.

\section{Introduction}

Children are exposed to screen based media from infancy and by early childhood are proficient users or consumers of television, computers and an array of mobile screen devices. Its pacifying effect on children is valued by many parents, with devices entertaining children in or outside the family home. ${ }^{1}$ However, excessive use of such entertainment is associated with numerous consequences to health and wellbeing, ${ }^{2}$ the nature and extent of which is dependent on the screen type and content. ${ }^{2,3}$ For instance, there is evidence of a doseresponse association between television viewing and body mass index (BMI) in children, ${ }^{4}$ while excessive online video gaming is associated with reduced attention, hyperactivity, poor prosocial behavior and conduct issues. ${ }^{5}$

Screen use is associated with impaired sleep in children, particularly, delayed onset of sleep and reduced sleep duration. ${ }^{3}$ Proposed mechanisms for sleep disruptions may include screen light exposure delaying the release of melatonin, interactive media content increasing arousal before bedtime and screen use deferring bedtime. ${ }^{6,7}$ This is of concern, given insufficient sleep is associated with behavioral problems, learning difficulties and poorer health outcomes in children. ${ }^{8,9}$ 
Emotional and behavioral problems are associated with excess screen time, including lower self-esteem and wellbeing, anti-social behavior, ${ }^{2}$ and reduced social skills. ${ }^{5}$ It is estimated that $60 \%$ of children aged five to eight years access the internet, and of these $80 \%$ play online games, ${ }^{10}$ highlighting the popularity of this activity among young children. Such associations may be explained by excessive screen time leading to reduced social interaction with peers, parents or significant others, poor sleep, cyber bullying ${ }^{11}$ and exposure to violent media. Moreover, exposure to media violence is associated with increased aggression. ${ }^{12}$ Heightened anxiety may also be experienced by children exposed to tragic events depicted in mass media. ${ }^{13}$

Furthermore, excess screen time is associated with poorer learning and academic achievement in children. Reduced capacity for concentration and attention is seen in children with excessive screen use, particularly excessive video gaming, ${ }^{14}$ mobile phone use ${ }^{15}$ or the early consumption of non-educational television programs. ${ }^{16}$ This may be explained through the displacement of activities that assist in skill development, or adaptation to the level of visual and auditory stimulation from a screen and increased multi-tasking. ${ }^{17}$

Limiting screen exposure in children is difficult to achieve, especially when considering background television. It is reported that mothers of infants have the television switched on in the same room in which the child plays at least $50 \%$ of the time. ${ }^{18}$ Background television has been reported to reduce the number of verbal exchanges and the quality of language employed by the parent when responding to the child, ${ }^{19}$ and that used between children. Additionally, background television adversely affects a child's ability to engage in play, such as reducing the duration a child interacts with one play item. This is of concern given the importance of play in the development of various cognitive skills such as language, ${ }^{20}$ attentional capacity, problem solving, and gross and fine motor development. ${ }^{18}$ It also risks exposure to age-inappropriate content. ${ }^{18}$

The American Academy of Pediatrics (AAP) guidelines recommend limiting screen use in children: a maximum of two hours of leisure viewing per day for children aged over five years, ${ }^{21}$ one hour per day for two to five year olds, no screen exposure for under one to two year olds, and that any television viewing occur with adult supervision. However the recommendations have been criticized as being unrealistic, with many parents using screens as a "baby sitter"14 and children's screen use well in excess of the guidelines. ${ }^{22}$ The guidelines were recently modified, encouraging health professionals to assist parents in developing media use plans, setting screen time limits and ensuring age-appropriate content. $^{23}$

The effectiveness of strategies to reduce screen time in children has been the subject of numerous systematic reviews. ${ }^{24-30}$ Maniccia et al. ${ }^{29}$ found that television control devices, television budgets, viewing plans and goal setting produced a small but significant effect on screen time reduction in children. Schmidt et al. ${ }^{31}$ also reported electronic monitoring 
devices to be effective, however raised concerns around low compliance and acceptability by families, also reported by Steeves et al. ${ }^{27}$ In contrast, Wu et al. ${ }^{28}$ found television monitoring devices not to be effective in reducing screen time, proposing that study participants replace television viewing with other forms of screen based behavior. Altenburg et al. ${ }^{32}$ found conflicting/insufficient evidence for many interventions targeting the reduction of sedentary behavior, with the exception of a 'television turn-off week'.

Reducing screen time is often embedded in an intervention targeting multiple health behaviors. Categorizing interventions into "screen time only" and "screen time plus" was an approach utilized by Ramsey Buchanan et al. ${ }^{24}$ to differentiate the two. "Screen time only" studies of high intensity and that utilized electronic monitoring devices were shown to produce greater reduction in screen time for children. This is in contrast to findings from $\mathrm{Wu}$ et $a l .{ }^{28}$ reporting that screen time reduction strategies embedded in health promotion curricula and supported with counselling are more successful in achieving screen time reduction. When considering school curriculum based programs, Friedrich et al. ${ }^{30}$ reported most interventions aiming to reduce sedentary behavior (screen time) target multiple health behaviors and demonstrate a significant reduction in screen time in school children, with programs encouraging family participation showing greater success. The importance of family involvement was also emphasized by Marsh et al. ${ }^{33}$ reporting intervention setting to be of little consequence, providing programs utilize strong family involvement, a result also reported by Schmidt et al. ${ }^{31}$

While various strategies to reduce screen time may be effective, they do not address screen content. Modifying screen content, such as replacing violent or fast-paced programs with age appropriate programs, has shown to improve behavior. ${ }^{34}$ Modifying content and providing media literacy education to mitigate the potential harm incurred from viewing violent content has also been well researched. In a narrative review of experimental and curriculum based media literacy interventions for reducing aggression, Cantor and Wilson ${ }^{35}$ found that commonly utilized strategies to mitigate the adverse affects of viewing mass media, such as co-viewing programs with children and providing commentary on content, produced mixed results, with the risk of indirectly endorsing the depicted behavior if commentary is insufficient. Study shortcomings were identified, with behavioral outcomes frequently including self or peer reports of aggression, or aggressive attitudes and lack long term follow-up. ${ }^{35}$ While media literacy interventions have been shown to be effective in raising awareness of a range of problematic and risk taking behavior, including violence, ${ }^{36}$ outcomes have focused on changing attitudes and few measure change in behavior.

Evaluating the effectiveness of interventions to improve sleep through controlling the use of screen based media is a relatively new field of research. Strategies being evaluated to improve sleep include the replacement of violent media or age-inappropriate content or restricting access to screens at bedtime. Primary research indicates that such education on screen use and healthy sleep practices in children has a positive effect. ${ }^{37}$ 
As the interrelated nature of screen use, sleep, obesity, and behavior is being better understood, more interventions are adopting a holistic approach and targeting multiple lifestyle behaviors ${ }^{38-40}$ such as sleep, diet, physical activity and screen use. The effectiveness of interventions to reduce screen time on $\mathrm{BMI}^{25,28,41}$ has been extensively reviewed. A limited number of reviews examining the effectiveness of media literacy programs on attitudes toward risk taking behavior ${ }^{35,36}$ and psychosocial interventions for school aged children's internet addiction, self-control and self-esteem ${ }^{42}$ have not specifically included controlling screen use as an outcome. Recent comprehensive systematic reviews have sought to determine the associations and health correlates with screen use, such as Carson et al. ${ }^{2}$ focusing on sedentary behavior, and Kostyrka-Allchorne et al. ${ }^{14}$ specifically addressing television viewing and its associations with language, play, executive function, academic performance and attention in children. This systematic review differs from KostyrkaAllchorne et al. ${ }^{14}$ as it seeks to determine the effect of health promotion intervention programs that control screen use and how this may impact on the outcomes of interest. No such studies were included in the systematic review by Carson et al. or Kosturka-Allchorne et al. A search of the JBI Database of Systematic Reviews and Implementation Reports, PubMed, Cochrane Library and PROSPERO has located no systematic review investigating the effects of intervention programs that include strategies to control screen use, including reducing total screen time (use of hand held devices, television or computer), time of viewing and/or content (social media, gaming or violent media) on sleep, behavioral and cognitive outcomes in children.

\section{Inclusion criteria}

\section{Participants}

This review will consider study populations that include children aged two to 14 years and/or their family, guardians or care givers. Exposure to screen based electronic media is not recommended for children under two years ${ }^{21}$ and this set the lower age cut-off. The upper age limit was set to include children in the final year of primary or middle school. Studies that specify an age range that overlaps with the current review cut-off must have at least $80 \%$ of participants within two to 14 years.

\section{Intervention}

This review will consider quantitative studies that evaluate intervention programs that include strategies to reduce screen time, control screen content or the time at which the content is viewed, regardless of intervention intensity or duration. Interventions may be carried out in any setting, such as school, child care centre, home or health clinic. The intervention may be directly targeted toward the child and or the family or primary care giver. Evaluating screen use may occur as part of a multi-faceted intervention program targeting behavior change to multiple lifestyle factors, such as diet, physical activity or sleep hygiene, which is an approach often used to prevent or address childhood obesity. 
Interventions targeting reduction in screen time may include reducing the number of screens within the home, reducing access, making access conditional to physical activity, utilizing screen viewing plans and goal setting, budgeting screen use or use of electronic monitoring devices.

Intervention programs specifically targeting sleep may include controlling screen use and developing routines or improving sleep hygiene.

Intervention programs aimed at improving behavior may, for example, include controlling screen use, swapping violent content with age appropriate content and providing education on media literacy.

\section{Exclusion criteria for interventions}

Interventions that do not include screen time as an outcome measure or changes to the amount of violent media viewed as an outcome measure will be excluded.

Interventions that do not incorporate education of the child or parent/guardian or include their involvement in performing the intervention, for example, limiting screen time, education on the impacts of violent media or screen use interfering with sleep patterns, will be excluded.

Interventions utilizing electronic media based strategies to improve sleep, health or participation in physical activity, without promoting reduction in screen time or changes to screen content or time of viewing, will be excluded, for example, when electronic media is used as a vehicle to deliver health promotion messages.

Interventions evaluating the effectiveness of a therapy provided through screen based electronic media will be excluded.

Interventions including pharmacological treatment, for example, those for internet addiction, will be excluded.

Studies that specifically include participants with a disability will be excluded, as their needs may not be representative of the general population.

\section{Comparator}

This review will include studies that compare the intervention to a passive control group, such as health promotion without screen related education, or active control group comparing one intervention for controlling screen use to another method.

\section{Outcomes}

This review will consider studies that include one or more of the following outcomes, measured by tools that are reliable and validated or considered appropriate and accepted for use in the relevant fields of research, examples of which may include and are not limited to: 
Sleep: sleep duration, bed time/sleep, as measured by Child Sleep Habits Questionnaire, ${ }^{43}$ Prospective Diary, or Actigraphy monitor. ${ }^{44}$

Cognitive skills: capacity for concentration and attention, executive function, engagement in play, school achievement and school performance, as measured by teacher report, school report cards, Tower of Hanoi task, ${ }^{45}$ Vineland Adaptive Behavior Scale, ${ }^{46}$ or play episode duration.

Behavior: aggression, antisocial behavior, prosocial behavior, hyperactivity, wellbeing, self-esteem, and depression, as measured by Strengths and Difficulties Questionnaire, ${ }^{47}$ Social Competence and Behavior Evaluation, ${ }^{48}$ and Child Behavior Checklist ${ }^{49}$ or Vineland Adaptive Behavior Scale.

Screen time hours, type of screen content viewed: as measured by Prospective Diary, Genre-based approach to measuring use of violent and non violent media, ${ }^{50}$ or Gentile's pathological video gaming scale. ${ }^{51}$

Secondary outcomes of interest include sleep disturbances, sleep deficit, day time sleepiness, as measured by Prospective diary, Child Sleep Habits Questionnaire, or Actigraphy monitor.

\section{Study types}

This review will consider experimental, quasi-experimental and prospective cohort interventional quantitative study designs including randomized controlled trials, nonrandomized controlled trials, before and after studies and interrupted time-series studies.

\section{Methods}

\section{Search Strategy}

The search strategy will aim to find both published and unpublished studies. An initial limited search of PubMed and PsycINFO has been undertaken followed by analysis of the text words contained in the title and abstract, and of the index terms used to describe the article. This informed the development of a search strategy which will be tailored for each information source. A full search strategy for PsycINFO is detailed in Appendix I. Search limits include studies published in English and no limit set for date of publication. The reference list of all studies selected for critical appraisal will be screened for additional studies.

\section{Information sources}

The databases to be searched include: PsycINFO, Embase, ERIC, Scopus and PubMed. The trial registers to be searched include: Cochrane Central Register of Controlled Trials, ClinicalTrials.gov and AustNZ trials register. A search for unpublished research will be completed in ProQuest Dissertations and Theses. A search of gray literature will be performed in Google. 


\section{Study Selection}

Following the search, all identified citations will be collated and uploaded into Endnote X7 (Clarivate Analytics, PA, USA) and duplicates removed. Titles and abstracts will then be screened for eligibility considering the inclusion criteria for this review. Studies that fulfil the inclusion criteria will be retrieved in full and their details imported into Joanna Briggs Institute System for the Unified Management, Assessment and Review of Information (JBI SUMARI). The full text of retrieved studies will be assessed in detail against the inclusion criteria by the author. Full text studies that do not meet the inclusion criteria will be excluded and reasons for exclusion will be reported. The results of the study location and inclusion process will be presented in a PRISMA flow diagram.

\section{Assessment of methodological quality}

Selected studies will be critically appraised by two independent reviewers at the study level for methodological quality in the review using the standardized critical appraisal instruments from the Joanna Briggs Institute for the following study types randomized control trials, quasi-experimental and cohort studies. ${ }^{51}$ All studies, regardless of their methodological quality, will undergo data extraction and synthesis (where possible).

\section{Data extraction}

Data will be extracted from papers included in the review using the standardized data extraction tool available in JBI SUMARI ${ }^{51}$ by two independent reviewers. The data extracted will include specific details about the interventions, populations, study methods and outcomes of significance to the review question and specific objectives. If possible, data from studies that include participants within and outside the age range will be considered, where the results are categorized according to age and data can be extracted for the age group of interest. Missing or additional data will be requested of the study author, where required.

\section{Data synthesis}

For the purposes of data synthesis, studies will be grouped according to both outcome definition and intervention type such as "Screen use only" or "screen use plus" (multifaceted intervention). Depending on the characteristics of included studies, results will be stratified according to age, gender, type of screen, program duration and intensity. If data allows, comparisons may be made between intervention setting, for example, school based or home based, the level of family involvement, intensity of intervention delivery or duration. If the included studies are sufficiently homogenous, data will be pooled in statistical meta-analysis using JBI SUMARI. Effect sizes will be expressed as weighted (or standardized) mean differences (for continuous data) and their $95 \%$ confidence intervals will be calculated for analysis. A random effects model will be used and heterogeneity will be assessed statistically using the standard chi-squared and I squared tests. Separate analysis of data based on a studies methodological quality will be performed to determine the impact of identified bias or study design on results. 
Depending on the characteristics of included studies, results will be stratified according to age, gender, program duration and intensity for subgroup analyses where there is sufficient data to do so. If possible, data from studies that include participants within and outside the age range will be considered, where the results are categorized according to age and data can be extracted for the age group of interest. Where statistical pooling is not possible, the findings will be presented in narrative form including tables and figures to aid in data presentation where appropriate.

A funnel plot will be generated to assess publication bias if there are 10 or more studies included in a meta-analysis. Statistical tests for funnel plot asymmetry (Egger test, Begg test, Harbord test) will be performed, where appropriate.

\section{Assessing confidence}

A Summary of Findings will be created using GRADEPro GDT software. ${ }^{52}$ The Grading of Recommendations Assessment, Development and Evaluation (GRADE) approach for grading the quality of evidence will be followed. The Summary of Findings will present the following information where appropriate: absolute risks for treatment and control, estimates of relative risk, and a ranking of the quality of the evidence based on study limitations (risk of bias), indirectness, inconsistency, imprecision and publication bias.

The following outcomes will be included in the Summary of Findings: sleep duration, sleep onset, capacity for attention and concentration, hyperactivity, aggression and antisocial/prosocial behavior, wellbeing, depression, self-esteem, executive function, school performance and achievement, and play

\section{Acknowledgements}

The author would like to acknowledge the contribution of Maureen Bell, University of Adelaide Librarian, in the development of the search strategy.

\section{References}

1. Nevski E, Siibak A. The role of parents and parental mediation on 0-3-year olds' digital play with smart devices: Estonian parents' attitudes and practices. Early Years 2016;36(3):227-41.

2. Carson V, Hunter S, Kuzik N, Gray CE, Poitras VJ, Chaput J-P, et al. Systematic review of sedentary behaviour and health indicators in school-aged children and youth: an update. Appl Physiol Nutri Me 2016;41(6 Suppl 3):S240-65.

3. Hale L, Guan S. Screen time and sleep among school-aged children and adolescents: A systematic literature review. Sleep Med Rev 2015;21:50-8.

4. Biddle SJ, García Bengoechea E, Wiesner G. Sedentary behaviour and adiposity in youth: a systematic review of reviews and analysis of causality. Int J Behav Nutr Phy 2017;14(1):43.

5. Allen MS, Vella SA. Screen-based sedentary behaviour and psychosocial well-being in childhood: Cross-sectional and longitudinal associations. Ment Health Phys Act 2015;9:41-7. 
6. Lange K, Cohrs S, Skarupke C, Gorke M, Szagun B, Schlack R. Electronic media use and insomnia complaints in German adolescents: gender differences in use patterns and sleep problems. J Neural Transm (Vienna) 2017;124(Suppl 1):79-87.

7. Akacem L. Understanding the circadian physiology in early childhood: The role of napping and light at night. Dissertation Abstracts International: Section B: The Sciences and Engineering 2017;78:.(3-B(E)).

8. Hiscock H, Canterford L, Ukoumunne OC, Wake M. Adverse Associations of Sleep Problems in Australian Preschoolers: National Population Study. Pediatrics 2007;119(1):86-93.

9. Cespedes EM, Rifas-Shiman SL, Redline S, Gillman MW, Peña M-M, Taveras EM. Longitudinal associations of sleep curtailment with metabolic risk in mid-childhood. Obesity 2014;22(12):2586-92.

10. Australian Bureau of Statistics. 4901.1 Children's Participation in Cultural and Leisure Activities, Australia, April 2009. 2009.

11. Leung L. Predicting Internet risks: a longitudinal panel study of gratifications-sought, Internet addiction symptoms, and social media use among children and adolescents. Health Psychol Behav Med 2014;2(1):424-39.

12. Coker TR, Elliott MN, Schwebel DC, Windle M, Toomey SL, Tortolero SR, et al. Media Violence Exposure and Physical Aggression in Fifth-Grade Children. Acad Pediatr 2015;15(1):82-8.

13. Otto MW, Henin A, Hirshfeld-Becker DR, Pollack MH, Biederman J, Rosenbaum JF. Posttraumatic stress disorder symptoms following media exposure to tragic events: Impact of $9 / 11$ on children at risk for anxiety disorders. J Anxiety Disord 2007;21(7):888-902.

14. Kostyrka-Allchorne K, Cooper NR, Simpson A. The relationship between television exposure and children's cognition and behaviour: A systematic review. Dev Rev 2017;44:19-58.

15. Zheng F, Gao P, He M, Li M, Wang C, Zeng Q, et al. Association between mobile phone use and inattention in 7102 Chinese adolescents: a population-based cross-sectional study. BMC Public Health 2014;14(1):1022.

16. Zimmerman FJ, Christakis DA. Associations Between Content Types of Early Media Exposure and Subsequent Attentional Problems. Pediatrics 2007;120(5):986-92.

17. Giedd JN. The Digital Revolution and Adolescent Brain Evolution. J Adolescent Health: official publication of the Society for Adolescent Medicine 2012;51(2):101-5.

18. Masur EF, Flynn V, Olson J. The Presence of Background Television During Young Children's Play in American Homes. J Children Media 2015;9(3):349-67.

19. Kirkorian HL, Pempek TA, Murphy LA, Schmidt ME, Anderson DR. The Impact of Background Television on Parent-Child Interaction. Child Dev 2009;80(5):1350-9.

20. Frank Masur E, Flynn V, Olson J. Infants' background television exposure during play: Negative relations to the quantity and quality of mothers' speech and infants' vocabulary acquisition. First Language 2016;36(2):109-23.

21. Bar-on M, Broughton D, Buttross S, Corrigan S. Children, Adolescents, and Television. Pediatrics 2001;107(2):423-6. 
22. Houghton S, Hunter SC, Rosenberg M, Wood L, Zadow C, Martin K, et al. Virtually impossible: limiting Australian children and adolescents daily screen based media use. BMC Public Health 2015;15(1):5.

23. Hill D, Ameenuddin N, Reid Chassiakos Y, Cross C, Hutchinson J, Levine A, et al. Media and Young Minds. Pediatrics 2016;138(5).

24. Ramsey Buchanan L, Rooks-Peck CR, Finnie RK, Wethington HR, Jacob V, Fulton JE, et al. Reducing Recreational Sedentary Screen Time: A Community Guide Systematic Review. Am J Prev Med 2016;50(3):402-15.

25. Wahi G, Parkin PC, Beyene J, Uleryk EM, Birken CS. Effectiveness of interventions aimed at reducing screen time in children: A systematic review and meta-analysis of randomized controlled trials. Arch Pediatr Adolesc Med 2011;165(11):979-86.

26. Schmidt ME, Haines J, O’Brien A, McDonald J, Price S, Sherry B, et al. Systematic Review of Effective Strategies for Reducing Screen Time Among Young Children. Obesity 2012;20(7):1338-54.

27. Steeves JA, Thompson DL, Bassett DR, Fitzhugh EC, Raynor HA. A review of different behavior modification strategies designed to reduce sedentary screen behaviors in children. J Obes 2012;2012:Article ID 379215.

28. Wu L, Sun S, He Y, Jiang B. The effect of interventions targeting screen time reduction: A systematic review and meta-analysis. Medicine 2016;95(27):e4029.

29. Maniccia DM, Davison KK, Marshall SJ, Manganello JA, Dennison BA. A metaanalysis of interventions that target children's screen time for reduction. Pediatrics 2011;128(1):E193(17).

30. Friedrich RR, Polet JP, Schuch I, Wagner MB. Effect of intervention programs in schools to reduce screen time: a meta-analysis. J Pediatr (Rio J) 2014;90(3):232-41.

31. Schmidt ME, Haines J, O’Brien A, McDonald J, Price S, Sherry B. Systematic review of effective strategies for reducing screen time among young children. Obesity (Silver Spring) 2012;20(7):1338-54.

32. Altenburg TM, Kist-van Holthe J, Chinapaw MJM. Effectiveness of intervention strategies exclusively targeting reductions in children's sedentary time: a systematic review of the literature. Int J Behav Nutr Phy 2016;13(1):65.

33. Marsh S, Foley LS, Wilks DC, Maddison R. Family-based interventions for reducing sedentary time in youth: a systematic review of randomized controlled trials. Obes Rev 2014;15(2):117-33.

34. Christarkis D, Garrison M, Herrenkohl T, Haggerty $\mathrm{K}$, Rivara F, Zhou C, et al. Modifying media content for pre-school children: a randomised controlled trial. Pediatrics 2013;131(3):431-8.

35. Cantor J, Wilson BJ. Media and Violence: Intervention Strategies for Reducing Aggression. Media Psychology 2003;5(4):363-403.

36. Jeong S-H, Cho H, Hwang Y. Media Literacy Interventions: A Meta-Analytic Review. J Commun 2012;62(3):454-72.

37. Garrison MM, Christakis DA. The Impact of a Healthy Media Use Intervention on Sleep in Preschool Children. Pediatrics 2012;130(3):492-9.

38. Yoong SL, Chai LK, Williams CM, Wiggers J, Finch M, Wolfenden L. Systematic review and meta-analysis of interventions targeting sleep and their impact on child body mass index, diet, and physical activity. Obesity 2016;24(5):1140-7. 
39. Haines J, McDonald J, O'Brien A, Sherry B, Bottino CJ, Schmidt ME. Healthy Habits, Happy Homes: randomized trial to improve household routines for obesity prevention among preschool-aged children. JAMA Pediatr 2013;167(11):1072-9.

40. Olsen NJ, Rohde JF, Heitmann BL. The Healthy Start project: a randomized, controlled intervention to prevent overweight among normal weight, preschool children at high risk of future overweight Jeanett Friis Rohde. Eur J Public Health 2015;25(Issue suppl 3):ckv174.067-7.

41. van Grieken A, Ezendam NP, Paulis WD, van der Wouden JC, Raat H. Primary prevention of overweight in children and adolescents: a meta-analysis of the effectiveness of interventions aiming to decrease sedentary behaviour. Int J Behav Nutr Phy 2012;9(1):61.

42. Yeun YR, Han SJ. Effects of Psychosocial Interventions for School-aged Children's Internet Addiction, Self-control and Self-esteem: Meta-Analysis. Healthcare Informatics Research 2016;22(3):217-30.

43. Owens JA, Spirito A, McGuinn M. The Children's Sleep Habits Questionnaire (CSHQ): psychometric properties of a survey instrument for school-aged children. Sleep 2000;23(8):1043-51.

44. O'Driscoll DM, Foster AM, Davey MJ, Nixon GM, Horne RSC. Can actigraphy measure sleep fragmentation in children? Arch Dis Child 2010;95(12):1031-3.

45. Welsh M, Satterlee-Cartmell T, Stine M. Towers of Hanoi and London: contribution of working memory and inhibition to performance. Brain Cogn 1999;41(2):231-42.

46. Sparrow S, Balla D, Cicchetu D. Vineland adaptive behaviour scales (survey form). Circle Pines, Minnesota, American Guidance Service;1984.

47. Goodman A, Goodman R. Population mean scores predict child mental disorder rates: validating SDQ prevalence estimators in Britain. J Child Psychol Psyc 2011;52(1):1008 .

48. LaFreniere P, Dumas J. Social Competence and Behavior Evaluationin Children Ages 3 to 6 years: The Short Form (SCBE-30). Psychol Assessment 1996;8(4):369-77.

49. Achenbach T. Intergrative guide for the 1991 CBCL/4-18, YSR and TRF profiles. 1991.

50. Busching R, Gentile D, Krahe B, Moller L, Khoo A, Walsh D, et al. Testing the reliability and validity of different measures of violent video game use in the United States, Singapore and Germany. Psychol Pop Media Cult 2015;4(2):97-111.

51. The Joanna Briggs Institute. Joanna Briggs Institute Reviewers' Manual: 2014 edition Australia: The Joanna Briggs Institute; 2014.

52. GRADEpro GDT: GRADEpro Guideline Development Tool [Software]. McMaster University, 2015 (developed by Evidence Prime, Inc.). Available from gradepro.org. 


\section{Chapter 3 The systematic review}

The following chapter presents the publication 'Interventions to control children's screen use and their effect on sleep: a systematic review and meta-analysis', ${ }^{19}$ submitted to the Journal of Sleep Research on 29 November 2019 and accepted for publication following peer review on 28 May 2020. The only modification to content was the reference to relevant content contained in the appendices presented in this thesis.

The Authors wish to acknowledge The Journal of Sleep Research. Article ID: JSR_13130; DOI: 10.1111/JSR.13130, reproduced with permission from Wiley. 


\title{
Interventions to control children's screen use and their effect on sleep: a systematic review and meta-analysis
}

\author{
Katie B. Martin ${ }^{1} \quad$ Jana M. Bednarz ${ }^{2} \quad$ Edoardo C. Aromataris ${ }^{1}$ \\ ${ }^{1}$ Joanna Briggs Institute, University of Adelaide, Adelaide, SA, Australia \\ ${ }^{2}$ School of Public Health, University of Adelaide, Adelaide, SA, Australia First \\ Correspondence: Katie B. Martin, Joanna Briggs Institute, University of Adelaide, 55 King William \\ Road, North Adelaide, SA 5006, Australia. Email: katie.martin@adelaide.edu.au
}

\begin{abstract}
Prolonged viewing of screen-based media is associated with poor sleep in children. Previous systematic reviews have analysed the effectiveness of interventions aiming to limit children's screen use; however, none have evaluated its effect on sleep. The aim of this systematic review was to evaluate the effect of interventions that incorporate strategies to control children's screen use on screen use and sleep. The databases Pubmed, Embase, Eric, Scopus and PsycInfo were searched during October 2017 and updated in February 2019 for experimental studies with a control that assessed interventions to control screen use in children aged 2-14 years and reported both screen use and sleep outcomes. From 3,872 initial records, 11 studies (six randomized control [RCT], four cluster RCT and one cluster, quasiexperimental) were eligible for inclusion. A total of 4,656 children aged 2-13 years were included in the studies. The mean reduction in screen time was $0.56 \mathrm{hr}$ (33 $\mathrm{min})$ /day (95\% confidence interval [CI], 0.92, 0.20) and the mean sleep duration increased by $0.19 \mathrm{hr}(11 \mathrm{~min}) /$ day $(95 \% \mathrm{CI}, 0.05,0.33)$. Bedtime was advanced by $0.16 \mathrm{hr}(10 \mathrm{~min})$ on weekdays and by $1.0 \mathrm{hr}$ at the weekend. Subgroup analyses indicated stronger intervention effects for interventions of shorter duration $(<3$ months), which specifically targeted screen use or sleep, and those with direct participant contact. In conclusion, small improvements in screen time and sleep duration can be achieved in children. It is not possible to determine if a reduction in screen time directly improves sleep, due to the limited number of studies, the presence of co-interventions, issues with studies' methodological quality and heterogeneity.
\end{abstract}

KEYWORDS: bedtime, electronic media, screen time, sleep duration, television

\section{1 | INTRODUCTION}

Children are increasingly proficient consumers of screen-based media, including television, computers and mobile devices. Excessive screen use, particularly in the evening, is associated with delayed sleep onset and reduced sleep duration in children (Carter, Rees, Hale, Bhattacharjee, \& Paradkar, 2016; Hale \& Guan, 2015; Yland, Guan, Emanuele \& Hale, 2015). Approximately $72 \%$ of American children have at least one electronic device in their 
bedroom overnight (National Sleep Foundation, 2014), the presence of which is associated with reduced sleep duration (Carter et al., 2016), increased bedtime resistance and other sleep problems (Cain \& Gradisar, 2010; Dube, Khan, Loehr, Chu, \& Veugelers, 2017). In addition, exposure to age-inappropriate content and viewing violent media may also impact sleep quality (Garrison, Liekweg, \& Christakis, 2011; Masur, Flynn, \& Olson, 2015). Proposed mechanisms for poorer sleep include: screen-light exposure delaying the release of melatonin and disrupting the circadian rhythm; interactive media content increasing arousal before bedtime; and screen use deferring bedtime (Akacem, 2016; Lange et al., 2017). The potential role of screen-based media in sleep insufficiency in children is of importance, given that inadequate sleep is associated with obesity (Garmy, Clauson, Nyberg \& Jakobsson, 2018), behavioural problems, learning difficulties and poorer health outcomes (Cespedes et al., 2014; Hiscock, Canterford, Ukoumunne, \& Wake, 2007).

Interventions to reduce screen time in children have been previously investigated (Friedrich, Polet, Schuch, \& Wagner, 2014; Maniccia, Davison, Marshall, Manganello \& Dennison, 2011; Marsh, Foley, Wilks, \& Maddison, 2014; Ramsey Buchanan et al., 2016; Schmidt, et al., 2012; Steeves, Thompson, Bassett, Fitzhugh, \& Raynor, 2011; Wahi, Parkin, Beyene, Uleryk, \& Birken, 2011; Wu, Sun, He, \& Jiang, 2016). Many of these interventions aimed to reduce body mass index (BMI) by addressing sedentary behaviour and used screen time as a proxy for sedentary behaviour. A review by Ramsey Buchanan et al. (2016) recommended that future studies and reviews evaluate additional outcomes that may be affected by modifying screen time, such as sleep. Indeed, as the interplay between screen use, sleep and obesity is being increasingly acknowledged (Garmy et al., 2018; Yoong et al., 2016), more interventions are targeting multiple lifestyle behaviours, including nutrition, screen use, physical activity and sleep (Haines et al., 2013; Maddison et al., 2014; Puder et al., 2011; Walton et al., 2015). Few interventions have focused exclusively on controlling screen use and improving sleep (Garrison \& Christakis, 2012). The objective of this systematic review was to evaluate the effect of interventions that incorporated strategies to control screen use, such as the effects of screen time, time of viewing or content viewed on children's sleep, including sleep duration, bedtime and sleep problems.

\section{2 | METHODS}

This systematic review was performed following PRISMA guidelines (Moher, Liberati, Tetzlaff, \& Altman, 2009) and the methodology of the Joanna Briggs Institute (JBI) 
(Aromataris \& Munn, 2017) according to an a priori protocol (Martin, Porritt, \& Aromataris, 2018) registered with PROSPERO, number CRD42018103204.

\subsection{Study Selection}

The population of interest was children aged between 2 and 14 years, as sleep outcomes in children under 2 years may be affected by a range of additional factors (Sadeh, Mindell, Luedtke, \& Wiegand, 2009), whereas 14 years reflects the age at which children finish primary or middle school. Studies were required to have evaluated an intervention provided to either the child and/or child's caregiver. Here, 'caregiver' refers to a parent, relative or person responsible for providing care for a child. All interventions addressing control of screen use (television [TV], computer and mobile devices), either on its own or as part of a broader health promotion programme, in any setting, were considered. Primary outcomes included total screen time (hr/day) and sleep duration (hr/day). Secondary outcomes were screen time in the hour before bedtime, time spent viewing specific screen devices, bedtime, bedtime resistance and sleep problems. Eligible studies used an experimental design with a control group, reported both screen use and sleep-related outcomes and were published in English.

\subsection{Search Strategy}

A systematic search of databases, including PubMed, PsycINFO, Embase, Eric, Scopus, and the Cochrane Central Register of Controlled Trials, and grey literature (Google) was conducted in October 2017 and updated in February 2019. Keywords related to the concepts "child", "electronic media", "intervention" and "sleep" were adapted for each database as needed. A full search strategy for PsycINFO is included in Appendix I.

All retrieved citations were uploaded into Endnote X7 (Clarivate Analytics) and duplicates removed. Titles were screened and abstracts assessed for eligibility (KM). The full text of potentially relevant studies was retrieved for final determination of eligibility $(\mathrm{KM})$.

\subsection{Assessment of methodological quality}

The methodological quality of eligible studies was independently assessed by two reviewers (KM and JL) utilizing the JBI critical appraisal tool for randomized controlled trials and quasi-experimental studies (Aromataris \& Munn, 2017) (See Appendix II). The tool was piloted to ensure consistency between reviewers. Any discrepancies were resolved through 
discussion; a third reviewer was not required. Overall study quality was assessed according to the extent to which each study met each criterion.

\subsection{Data extraction}

Data were extracted (KM) using a structured form (see Appendix III) tailored to prompt retrieval of relevant information, including the study's country of origin, study design, year of publication, participant age, sample size, intervention details (content, frequency of contact, duration, follow-up, delivery mode and setting) and screen use and sleep outcome measures. The author of one study was contacted via an email requesting total screen-time data, rather than date stratified according to screen type (TV, internet and videogaming); no response was received (Bickham, Hswen, Slaby, \& Rich, 2018) and only the data reported for $\mathrm{TV}$ viewing from this study were included in the analysis.

\section{5 | Data analysis}

Unadjusted mean differences for change in screen time (hr/day) and sleep duration (hr/day) from baseline to post-intervention between treatment groups were used in the analysis. Postintervention data from the time-point closest to intervention completion were used if there were multiple post-intervention time-points. Sustainability of the intervention effect was not analysed as only three studies collected relevant data, each at a different post-intervention time-point. Secondary outcomes were reported in a narrative synthesis.

Where studies reported mean values for sleep duration and screen time at pre-intervention and post-intervention time-points, point estimates and variance for mean change from baseline for each treatment group were obtained using the methods outlined by Harris, Kuramoto, Schulzer, and Retallack (2009) and Higgins and Green (2011) (See Appendix IV sections a and $b$ ). This required plausible correlation coefficients to be derived using data from existing studies that provided standard deviation data for both outcomes at baseline and post-intervention and for the mean change. Correlation coefficients of 0.34 and 0.37 were estimated for screen time and sleep duration, respectively, based on the values reported by a study from Haines et al. (2013) (See Appendix IV section a). Previous reviews on screen time used correlation coefficients of 0.5 (Wu et al., 2016) and 0.4-0.7 (Wahi et al., 2011). A sensitivity analysis showed that altering the correlation coefficients for screen time to 0.50 and to 0.54 for sleep duration had negligible effects on the results (data not reported). Standard deviations were calculated from the relevant $95 \%$ confidence interval (CI) in instances where they were not provided (Higgins \& Green, 2011) (See Appendix IV section

c). Formulas for combining subgroups were applied for studies reporting data stratified 
according to gender or school-year level (Higgins \& Green, 2011). Where studies provided separate screen-time hours according to weekday and weekend viewing (Bickham et al., 2018; Hammersley, Okely, Batterham, \& Jones, 2019) weekday viewing hours were used in the analysis. In summary, for the pooled analysis the outcomes of TV, weekday and total screen time (total time viewing all screen-based media) (hr/day) were combined, whereas weekend screen-time hours; videogame hours and internet hours (unless included in total screen time) were included in a narrative analysis. Of the five cluster-randomized trials, only one reported that clustering had been accounted for in the analysis (Puder et al., 2011), otherwise, this was not reported explicitly (Tamura \& Tanaka, 2014; Walton et al., 2015; Wolfson, Harkins, Johnson, \& Marco, 2015), and information required to either inflate standard errors or adjust effective sample size was inadequate.

Meta-analysis for mean difference in mean change from baseline between treatment groups was performed using the generic inverse variance method using Review Manager Software, V5.2 (The Nordic Cochrane Centre, Copenhagen, Denmark). Random effects were specified due to expected between-study and within-study differences (Higgins \& Green, 2011). As high heterogeneity has been consistently reported in meta-analyses of screen-time-reduction interventions (Wahi et al., 2011; Wu et al., 2016), the $I^{2}$ test was applied to quantify variability (Higgins \& Green, 2011). Subgroup analyses were performed to explore potential causes of heterogeneity and how the intervention effect varied according to the pre-specified participant-related factors, such as target age group and recipient (child or caregiver) or intervention characteristics, such as intervention focus (multiple lifestyle behaviours, screen use or sleep education only), duration, setting and delivery (face to face or indirect/online) (Martin et al., 2018). Further sensitivity analyses were conducted to test the robustness of intervention effect, with the removal of outliers and sequential elimination of individual studies.

Publication bias was unable to be assessed formally due to the small number of included studies (Higgins \& Green, 2011).

\section{3 | RESULTS}

\section{1 | Study identification and inclusion}

Following the updated search, 3,872 records were retrieved. After removal of duplicates, 2,511 titles were screened and 162 abstracts reviewed against eligibility criteria, from which 43 full-text articles were retrieved and reviewed for inclusion (Figure 1). Reasons for 
exclusion at the full-text stage were that the study was not published in English (two studies); the outcomes of interest were not measured (12 studies), participants were not of eligible age (11 studies), a control group was not included (four studies), description of the intervention was insufficient (two studies) and duplication (See Appendix V). Finally, 11 studies were included in this review (Bickham et al., 2018; Garrison \& Christakis, 2012; Haines et al., 2013; Hammersley et al., 2019; Krossbakken et al., 2018; Maddison et al., 2014; Puder et al., 2011; Tamura \& Tanaka, 2014; Tomayko et al., 2018; Walton et al., 2015; Wolfson et al., 2015).

\section{2 | Study characteristics}

Characteristics of the included studies are reported in Table 1. Participants were aged 2-6 years in six studies, and 8-13 years in five studies. Children aged 7 years were not represented in any of the included studies. Sample sizes ranged from 54 to 1,657 participants for a total of 4,656 at the point of randomization. Studies reported either TV viewing hours (Haines et al., 2013; Walton et al., 2015) or total screen time based on all electronic media devices (Puder et al., 2011; Maddison et al., 2014; Tomayko et al., 2019); with some separating results for weekday and weekend viewing (Bickham et al., 2018; Haines et al., 2013; Hammersley et al., 2019) and one reporting each screen device/type (videogame/internet/TV) separately (Bickham et al., 2018); or videogame time only (Krossbakken et al., 2018). Mean daily screen-time hours ranged from $1.0 \mathrm{hr}$ to $2.8 \mathrm{hr} /$ day at baseline, with the exception of one study reporting over $4 \mathrm{hr}$ average viewing/day (Maddison et al., 2014). Mean night-time sleep duration at baseline ranged from 9.8 to 11.2 $\mathrm{hr}$ for children aged 2-6 years, and from 8.2 to $10.6 \mathrm{hr}$ for children aged 8-13 years. The primary outcome in studies that targeted weight-related behaviour change was BMI, with screen time and sleep duration reported as secondary outcomes (Hammersley et al., 2019; Maddison et al., 2014; Puder et al., 2011; Tomayko et al., 2019; Walton et al., 2015). Screen time was the primary outcome in one study (Haines et al., 2013). No studies reported any harm associated with the interventions. 


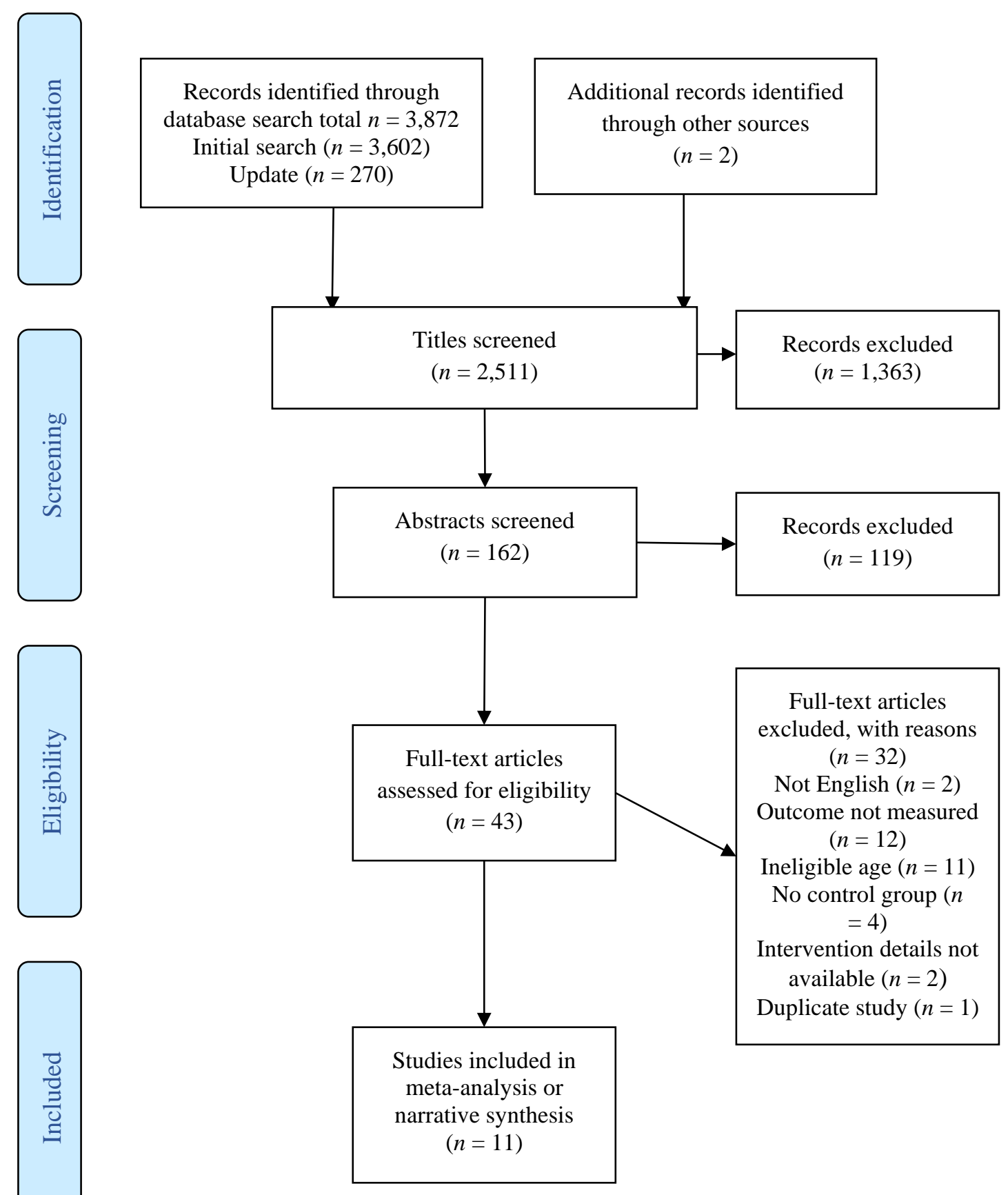

FIGURE 1 PRISMA flow diagram detailing process of study identification and inclusion 
TABLE 1 Characteristics of included studies

\begin{tabular}{|c|c|c|c|c|c|c|c|c|c|}
\hline \multirow[t]{2}{*}{ Author, year } & \multirow[t]{2}{*}{ Study design } & \multirow{2}{*}{$\begin{array}{l}\text { Age } \\
\text { Range, } \\
\text { y, } \\
\text { (mean) }\end{array}$} & \multirow[t]{2}{*}{$\begin{array}{l}\text { Sample } \\
\text { size }\end{array}$} & \multirow{2}{*}{$\begin{array}{l}\text { Location; } \\
\text { Demo- } \\
\text { graphic }\end{array}$} & \multirow{2}{*}{$\begin{array}{l}\text { Intervention } \\
\text { duration; } \\
\text { follow-up }\end{array}$} & \multirow[t]{2}{*}{$\begin{array}{l}\text { Setting; } \\
\text { recipient }\end{array}$} & \multicolumn{2}{|c|}{$\begin{array}{l}\text { Summary of intervention effect based on Authors' } \\
\text { results (measure used) }\end{array}$} & \multirow[t]{2}{*}{ Control } \\
\hline & & & & & & & Screen Use & Sleep & \\
\hline $\begin{array}{l}\text { Haines et al. } \\
(2013)\end{array}$ & RCT & $\begin{array}{l}2-5 \\
(4.1)\end{array}$ & 121 & $\begin{array}{l}\text { USA; } \\
\text { low income/ } \\
\text { ethnic } \\
\text { minority }\end{array}$ & 6 months; nil & $\begin{array}{l}\text { Home; } \\
\text { Caregiver \& } \\
\text { child }\end{array}$ & $\begin{array}{l}\text { Significant } \downarrow \text { weekend TV } \\
\text { viewing } \\
\downarrow \text { weekday TV viewing (PQ) }\end{array}$ & $\begin{array}{l}\uparrow \text { sleep duration by } 0.56 \\
\mathrm{hr} \\
(\mathrm{PQ})\end{array}$ & $\begin{array}{l}\text { Information on } \\
\text { developmental } \\
\text { milestones }\end{array}$ \\
\hline $\begin{array}{l}\text { Walton et al. } \\
\text { (2015) }\end{array}$ & $\begin{array}{l}\text { RCT } \\
\text { Pilot } \\
\text { Cluster }\end{array}$ & $2-5(3)$ & 54 & Canada & 9 weeks; nil & $\begin{array}{l}\text { Community } \\
\text { centre; } \\
\text { Caregiver \& } \\
\text { child }\end{array}$ & $\downarrow \mathrm{TV}$ viewing as (PQ) & $\begin{array}{l}\text { No effect on sleep } \\
\text { duration }(\mathrm{PQ})\end{array}$ & $\begin{array}{l}\text { Attention } \\
\text { matched } \\
\text { intervention } \\
\text { 'supervising for } \\
\text { home safety' }\end{array}$ \\
\hline $\begin{array}{l}\text { Garrison and } \\
\text { Christakis } \\
\text { (2012) }\end{array}$ & RCT & $3-5$ & 565 & USA & $\begin{array}{l}12 \text { months; } 6 \\
\text { months }\end{array}$ & $\begin{array}{l}\text { Home; } \\
\text { Caregiver }\end{array}$ & $\begin{array}{l}\downarrow \text { violent media content } \\
\text { (Media diary, prospective } \\
\text { PQ) }\end{array}$ & $\begin{array}{l}\text { Significantly lower odds } \\
\text { of "any sleep problem" } \\
\text { (CSHQ) }\end{array}$ & $\begin{array}{l}\text { Attention } \\
\text { matched } \\
\text { nutrition } \\
\text { intervention }\end{array}$ \\
\hline $\begin{array}{l}\text { Hammersley } \\
\text { et al. (2019) }\end{array}$ & $\mathrm{RCT}$ & $\begin{array}{l}2-5 \\
(3.5)\end{array}$ & 86 & Australia & $\begin{array}{l}11 \text { weeks; } \\
3 \text { months }\end{array}$ & $\begin{array}{l}\text { Online; } \\
\text { Caregiver }\end{array}$ & $\begin{array}{l}\downarrow \text { ST weekday and weekend } \\
\text { (PQ) }\end{array}$ & $\begin{array}{l}\uparrow \text { sleep duration } \\
\downarrow \text { sleep latency } \\
\text { (CSHQ; Actigraph } \\
\text { GT3X accelerometer) }\end{array}$ & $\begin{array}{l}\text { Fortnightly } \\
\text { emails with link } \\
\text { to parenting } \\
\text { website }\end{array}$ \\
\hline
\end{tabular}




\begin{tabular}{|c|c|c|c|c|c|c|c|c|c|}
\hline $\begin{array}{l}\text { Tomayko et } \\
\text { al. (2018) }\end{array}$ & $\begin{array}{l}\text { RCT } \\
\text { Modified } \\
\text { crossover* }\end{array}$ & $\begin{array}{l}2-5 \\
(3.6)\end{array}$ & 450 & $\begin{array}{l}\text { USA; } \\
5 \text { x AI } \\
\text { communities }\end{array}$ & $\begin{array}{l}12 \text { months; } \\
\text { nil }\end{array}$ & $\begin{array}{l}\text { Home/online } \\
\text { Caregiver }\end{array}$ & $\begin{array}{l}\text { No significant effect on ST } \\
\text { (PQ) }\end{array}$ & $\begin{array}{l}\text { No significant effect on } \\
\text { weekday or weekend } \\
\text { sleep duration (PQ) }\end{array}$ & $\begin{array}{l}\text { Attention } \\
\text { matched 'safety } \\
\text { journey' } \\
\text { intervention }\end{array}$ \\
\hline $\begin{array}{l}\text { Puder et al. } \\
\text { (2011) }\end{array}$ & $\begin{array}{l}\text { RCT } \\
\text { Cluster } \\
\text { Adjusted for } \\
\text { clustering }\end{array}$ & $(5.2)$ & 652 & $\begin{array}{l}\text { Switzerland; } \\
\text { high } \\
\text { migrant } \\
\text { population; } \\
40 \text { x public } \\
\text { pre-school } \\
\text { classes }\end{array}$ & $\begin{array}{l}10 \text { months; } \\
\text { nil }\end{array}$ & $\begin{array}{l}\text { School; } \\
\text { Caregiver \& } \\
\text { child }\end{array}$ & $\downarrow$ ST (PQ) & $\begin{array}{l}\text { No significant effect on } \\
\text { sleep duration }(\mathrm{PQ})\end{array}$ & $\begin{array}{l}\text { Regular } \\
\text { curriculum; one } \\
\text { parent info } \\
\text { session }\end{array}$ \\
\hline $\begin{array}{l}\text { Krossbakken } \\
\text { et al. (2018) }\end{array}$ & $\begin{array}{l}\text { RCT } \\
\text { Post } \\
\text { intervention } \\
\text { comparison }\end{array}$ & $\begin{array}{l}8-13 \\
(10.1)\end{array}$ & 1,657 & Norway & $\begin{array}{l}4 \text { months; } \\
\text { nil }\end{array}$ & Caregiver & $\begin{array}{l}\text { No effect on video game } \\
\text { problems, weekday or } \\
\text { weekend game time (Video } \\
\text { game problems DSM-5, } \\
\text { PQ) }\end{array}$ & $\begin{array}{l}\text { No effect on child sleep } \\
\text { problems or bedtime } \\
\text { resistance (CSHQ) }\end{array}$ & Non-intervention \\
\hline $\begin{array}{l}\text { Maddison et } \\
\text { al. (2014) }\end{array}$ & RCT & $\begin{array}{l}9-12 \\
(11.2)\end{array}$ & 251 & $\begin{array}{l}\text { New } \\
\text { Zealand; } \\
>15 \mathrm{~h} / \text { week } \\
\text { ST, } \\
\text { overweight }\end{array}$ & 20 weeks; nil & $\begin{array}{l}\text { Home; } \\
\text { Caregiver \& } \\
\text { child }\end{array}$ & $\begin{array}{l}\downarrow \text { ST (Multimedia Activity } \\
\text { Recall for Children and } \\
\text { Adolescents (child report) }\end{array}$ & $\begin{array}{l}\text { Nil change in sleep } \\
\text { duration (Child report) }\end{array}$ & $\begin{array}{l}\text { Waitlist non- } \\
\text { intervention }\end{array}$ \\
\hline $\begin{array}{l}\text { Wolfson et al. } \\
(2015)\end{array}$ & $\begin{array}{l}\text { RCT } \\
\text { Cluster }\end{array}$ & $12 \mathrm{y}$ & 143 & $\begin{array}{l}\text { USA; } \\
2 \text { x public } \\
\text { urban } \\
\text { middle } \\
\text { schools }\end{array}$ & $\begin{array}{l}4 \text { weeks; } \\
8 \text { months }\end{array}$ & $\begin{array}{l}\text { School; } \\
\text { Child }\end{array}$ & $\begin{array}{l}\downarrow \text { BT screen use by } 5 \text { mins } \\
\text { (Child report) }\end{array}$ & $\begin{array}{l}\text { Significant improvement } \\
\text { in sleep hygiene } \\
\text { practices; } \uparrow \text { sleep } \\
\text { duration on weekday by } \\
13 \text { min; }\end{array}$ & $\begin{array}{l}\text { Regular } \\
\text { curriculum and } \\
\text { 'research on } \\
\text { adolescent sleep } \\
\text { patterns' }\end{array}$ \\
\hline
\end{tabular}


Earlier BT (SSHQ \&

ASHS)

\begin{tabular}{|c|c|c|c|c|c|c|c|c|c|}
\hline $\begin{array}{l}\text { Tamura and } \\
\text { Tanaka } \\
(2014)\end{array}$ & $\begin{array}{l}\text { RCT } \\
\text { Cluster }\end{array}$ & $\begin{array}{l}\text { Year } 4, \\
5 \text { and } \\
6 \\
\text { classes }\end{array}$ & 148 & $\begin{array}{l}\text { Japan; } \\
2 \text { x public } \\
\text { elementary } \\
\text { schools }\end{array}$ & 2 weeks; nil & $\begin{array}{l}\text { School; } \\
\text { Child }\end{array}$ & $\begin{array}{l}\text { Significant } \uparrow \text { in students } \\
\text { limiting screen use before } \\
\text { BT (Child report) }\end{array}$ & $\begin{array}{l}\uparrow \text { sleep duration and } \\
\text { earlier BT (Child } \\
\text { report) }\end{array}$ & $\begin{array}{l}\text { Regular } \\
\text { curriculum; } \\
\text { Waitlist non- } \\
\text { intervention }\end{array}$ \\
\hline $\begin{array}{c}\text { Bickham et } \\
\text { al. (2018) }\end{array}$ & $\begin{array}{l}\text { Quasi- } \\
\text { experimental } \\
\text { Cluster } \\
\text { (adjusted } \\
\text { data)** }\end{array}$ & $12 \mathrm{y}$ & 479 & $\begin{array}{l}\text { USA; } \\
2 \text { x rural } \\
\text { middle } \\
\text { schools }\end{array}$ & $\begin{array}{l}6 \text { weeks; } \\
\text { Nil }\end{array}$ & $\begin{array}{l}\text { School; } \\
\text { Child }\end{array}$ & $\begin{array}{l}\downarrow \text { weekday TV by } 17 \text { min; } \\
\downarrow \text { weekend TV; } \downarrow \\
\text { background TV; significant } \\
\downarrow \text { weekend internet by } \\
22 \text { min; no change in } \\
\text { weekday Videogaming } \\
\text { (child report) }\end{array}$ & $\begin{array}{l}\text { Significant } \uparrow \text { sleep } \\
\text { duration compared to } \\
\text { control } \\
\text { Sleep duration } \uparrow \text { by } 10 \\
\text { min (child report) }\end{array}$ & $\begin{array}{l}\text { Regular } \\
\text { curriculum; } \\
\text { waitlist, non- } \\
\text { intervention }\end{array}$ \\
\hline
\end{tabular}

Note: Studies are listed according to participant age.

Sample size at baseline.

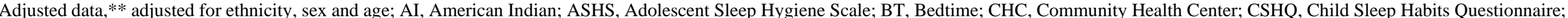

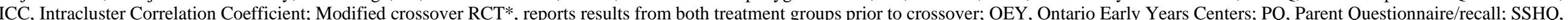

School Sleep Habits Questionnaire; ST, Screen Time; TV, Television. 
TABLE 2 Details of interventions to control screen use and improve sleep in children aged 2-13 years

\section{Intervention Description (ref)}

Healthy Habits, Happy Homes (Haines et al., 2013)

Aim: Improve household routines to prevent overweight

Creating healthy family routines, focusing on family mealtimes, adequate sleep and limiting TV.

Specific content: Unit 1: "Routines": creating a daily schedule; "sleep for better health"; sit at table for meals, TV off during meals. Evening routine: “3 B's" bath, book, bed; Unit 2: sleep. sleep tips, limiting ST in evening, "good night moon" book, quite time, soft singing; Unit 3: Limiting inactivity and ST; Unit 4: "Eating better". Healthy snacking, cooking together, education on sugar-sweetened beverages.

Environment: Removal of TV from child's bedroom, provision of relevant activities and games.

Parental skills: Importance of parental role modelling

Parents and Tots Together (Walton et al., 2015)

Aim: Changing general parenting behaviour and improving weight related behaviors

Obesity prevention through promoting family PA, bedtime routines, limiting ST, nutrition. Approx. $65 \%$ of the programme focused on general parenting and $35 \%$ on weightrelated behaviour.

Specific content: Topics included: Child-centred time, being physically active with your child, importance of family and bedtime routines; alternatives to using food as a reward; limiting ST; identifying child's hunger and satiety cues, reducing sugar-sweetened drink intake; family-based physical activities; problem-solving with caregivers about child's health behaviours.

Parental skills: Appropriate praise and rewards, setting boundaries, stress management, improving parental selfefficacy and warmth

Healthy Media Use Intervention (Garrison \& Christakis, 2013)

Aim: replace violent media content with age appropriate prosocial and educational content

Educate caregivers in substituting violent media content with age-appropriate pro-social and educational content, education on TV ratings and guidelines.

Specific content: Utilized motivational interviewing, goal setting and problem solving. Education on co-viewing, discussing media content with the child, raise awareness of media content consumed. Providing examples of specific ageappropriate, pro-social and educational shows/content

Parental skills: Improve parental self-efficacy regarding media choices for their children

Time 2b Healthy (Hammersley et al., 2019)

Aim: Improve weight related behaviours

\section{Delivery}

Each unit implemented in person in the home with individually tailored counselling; motivational coaching by specifically trained "health educator" Caregiver received:

$4 \mathrm{x}$ home visits;

$4 \times$ coaching phone calls;

$2 \mathrm{x}$ text messages/week for the first 16 weeks, then weekly for last 8 weeks;

$4 \mathrm{x}$ mailed, unit-related educational material

\section{Child received:}

$4 \mathrm{x}$ activity packs and incentives

Financial Incentives:

$\$ 40$ received at baseline data collection, $\$ 50$ at completion

\section{Caregiver involvement:}

$1 \times 2 \mathrm{hr}$ /week parent group discussion session (9 in total) at community centre conducted by group facilitator trained in the programme

Printed educational information

Child involvement:

Children attended session concurrent to above

Financial Incentives:

Meal and bus fare to attend sessions $\$ 20$ gift card received at each data collection visit, entry into $\$ 100$ raffle for the 9-month time point

\author{
Caregiver involvement: \\ $1 \mathrm{x}$ home visit; \\ monthly phone calls delivered by \\ specifically trained case manager; \\ $6 \mathrm{x}$ DVDs containing 5-10min \\ examples of appropriate children's \\ shows; \\ monthly mailings of programme guide \\ tailored to the family and newsletter \\ with tips and reinforcement \\ Child involvement: \\ None \\ Financial Incentives: \\ None
}

Caregiver involvement:

$6 \mathrm{x}$ online modules; 
Targeted multiple lifestyle behaviours, addressing nutrition $(\mathrm{n}=$ 2), PA, ST and sleep.

Specific content: Each module included online reading material, videos, activities, quizzes and goal setting. Participants received individualized feedback on their goals at end of each module and provided with advice to improve goals using the SMART goal framework. Participants encouraged to post photos recipes, personal experiences on program Facebook site relevant to each module. Parents were able to email or phone if they had any questions or concerns

Healthy Children, Strong Families 2 (Tomayko et al., 2018)

Aim: Improve obesity associated health behaviours

Healthy lifestyle/obesity prevention for American Indian families. Participants in the "wellness journey" group received education focusing on: nutrition $(\mathrm{n}=3)$, PA, reducing ST, improving sleep habits and fostering family interaction.

Supportive materials included games, pedometers, recipes and a children's book for each of the target behaviours.

Parental skills: Stress reduction/management.

The Ballabeina Study (Puder et al., 2011)

Aim: Increase fitness and reduce overweight

Intervention focusing on four lifestyle behaviours: PA, nutrition, including "turn off the screen while you eat", limiting media use and the importance of sufficient sleep.

Environment: The built environment of the preschool class was adapted to promote PA, such as new mobile play equipment weekly email reminders to participate in online activities;

closed Facebook group participation, monitored by a dietician

\section{Child involvement:}

None

Financial Incentives:

One post from each online module received a gift card

\section{Caregiver involvement:}

Lesson 1 delivered by site coordinator in person.

12 x Monthly mailed "tool kit" of educational lessons and activities, supportive materials;

facebook group discussion for parents;

$2 \mathrm{x}$ weekly text messages

Child involvement:

Each "tool kit" included children's books and activities

Financial Incentives:

$\$ 50$ gift voucher for each data collection visit

\section{Caregiver involvement:}

$3 \mathrm{x}$ Interactive group sessions conducted by trained health promoters;

brochures, activity cards and worksheets brought home by children

\section{Child involvement:}

$4 \mathrm{x}$ playful PA programs per week developed by an exercise physiologist, trained health promoters and teachers;

$22 \mathrm{x}$ health promotion sessions at school

Financial Incentives:

None

\section{A parental guide for prevention of problematic video} gaming in children (Krossbakken et al., 2018)

\section{Aim: Improve videogaming behaviour}

Education on preventing problematic video gaming using simple guidebook developed based on clinical recommendations, treatment manual for videogaming addiction, factors identified as useful for preventing videogaming addiction and feedback from a parental reference group. Strategies included offering alternative activities to videogaming, no gaming during mealtimes, avoid video gaming before bedtime, addressing game content, strategies for limiting game time, videogame-free days.

Environment: Screen-free bedrooms, videogaming occurring in a common room, router access restrictions

Parental skill: Importance of parental role modelling

\section{Caregiver involvement:}

1 x Guidebook (hard copy, mailed) Child involvement:

None

Financial Incentives:

Enrolled in raffle with prizes including one of 50 gift vouchers and two iPad's, upon completion of questionnaire 
The Screen Time Weight Loss Intervention Targeting

Children at Home (SWITCH) Study (Maddison et al., 2014)

Aim: Decreasing leisure time sedentary screen-based behaviour

Reducing leisure time screen-based sedentary behaviour, through implementing changes to the home and family environment, provision of behaviour-change strategies, assistance to budget media time, strategies to reduce ST, alternative activities to screen-based media and an activity pack for children.

Environment: "Time Machine" TV monitoring device, with 30 tokens, each token allowing $30 \mathrm{~min}$ of viewing.

Parental skill: Importance of parental role modelling
Caregiver involvement:

$1 \mathrm{x}$ Home visit for overview of the intervention by trained community worker;

monthly newsletter outlining strategies to reduce ST;

access to a secure website with information to support reducing ST and links to community-based activity programmes;

$2 \mathrm{x}$ time machine TV monitoring devices

\section{Child involvement:}

Activity pack of non-screen-based activity ideas and games

Financial Incentives:

None

Young Adolescent 'Sleep Smart' (Wolfson et al., 2015)

Aim: Improve sleep and sleep hygiene

Education and goal setting on sleep behaviours, sleep hygiene practices including limiting screen use before bed, using goal setting, roleplaying, educational games, and self-monitoring. Child received session related incentives (e.g., water bottle, pen, wallet sized cards with sleep hygiene principles).

\section{Caregiver involvement:}

Weekly newsletter related to each session so caregiver can reinforce sleep strategies

Child involvement:

8 x 40-min small group (9-11 participants) sleep hygiene education sessions facilitated by 2 trained BAlevel leaders (supervised by a licensed clinical psychologist)

Financial Incentives:

The child and their caregivers received a gift card for participating in the study
Sleep education and self-help treatment (Tamura \& Tanaka, 2014)

Aim: Improve sleep and knowledge of sleep related behaviour

Education on lifestyle rhythms and habits; strategies to improve sleep, including refraining from watching TV or videos before sleep; avoiding brightly lit places before sleep; regular sleepwake patterns; morning sunlight exposure; no napping; daily exercise.

Take the Challenge (Bickham et al., 2018)

Aim: Reduce ST and improve awareness of media content

Media education/reduction programme educating on health effects of excessive screen use and provide the experience of reducing ST. Specific content: Limiting ST, addressing snacking while viewing TV, ST self-management skills, critical evaluation of media content, identifying alternative activities to ST; monitoring own and peers reported media use, discussing research on media effects; completing media time budgeting forms.

\section{Caregiver involvement:}

None

Child involvement:

$1 \times 45$ min education session delivered at school by teacher in programme;

Child selects and monitors target

behaviour for two weeks

Financial Incentives:

None

\section{Caregiver involvement:}

Caregiver signed slips verifying if child was screen free on the previous day

\section{Child involvement:}

10-day school-wide screen-free challenge; education on screen media imbedded in school curriculum for 6 weeks, delivered by teachers trained in program

\section{Financial Incentives:}

None

Abbreviations: PA, Physical Activity; SMART, Specific, Measurable, Attainable, Realistic and Timely; ST, Screen Time; TV, Television. 


\section{3 | Intervention characteristics}

Details of interventions are reported in Table 2. Social cognitive theory informed intervention development in six studies (Bickham et al., 2018; Garrison \& Christakis, 2012; Hammersley et al., 2019; Maddison et al., 2014; Walton et al., 2015; Wolfson et al., 2015). The remaining studies did not specify an underlying theory for intervention development. Intervention duration ranged from 2 weeks to 12 months (median 4 months). Modes of delivery included direct (face-to-face) contact with caregivers in a group setting (Puder et al., 2011; Walton et al., 2015), at home with participation of the child (Haines et al., 2013; Maddison et al., 2014) or at home targeting caregivers only (Garrison \& Christakis, 2012). Four interventions were provided directly to students at school, of which one had direct caregiver involvement (Puder et al., 2011) and three did not (Bickham et al., 2018; Tamura \& Tanaka, 2014; Wolfson et al., 2015). Three studies evaluated interventions with no direct contact with participants. Two used online and interactive materials (Hammersley et al., 2019; Tomayko et al., 2019) and one study evaluated educational material delivered via mail (Krossbakken et al., 2018).

Interventions focused on screen-time reduction (Bickham et al., 2018; Krossbakken et al., 2018; Maddison et al., 2014), modification of screen content (Garrison \& Christakis, 2012), or limiting screen use before bedtime (Wolfson et al., 2015; Tamura \& Tanaka, 2014). Five studies targeted multiple lifestyle behaviours, including diet, physical activity, screen time and sleep (Haines et al., 2013; Hammersley et al., 2019; Puder et al., 2011; Tomayko et al., 2019; Walton et al., 2015). Studies with caregiver involvement included: encouraging role modelling of appropriate screen use, improving parental self-efficacy, such as through stress management strategies, teaching behaviour management skills and improving parental warmth. Other strategies included goal setting, motivational interviewing, peer review or self-monitoring of screen time.

Modifying the environment to facilitate behaviour change included screen-free bedrooms and mealtimes (Haines et al., 2013; Krossbakken et al., 2018; Puder et al., 2011), and providing alternative activities and resources such as games and books. Setting limits on screen use included "turning TV off when no one watching" (Haines et al., 2013), media budgeting (Bickham et al., 2018; Maddison et al., 2014), provision of a token-operated television control device (Maddison et al., 
2014); "screen-free challenges" (Bickham et al., 2018; Krossbakken et al., 2018) and limiting screen use before bedtime (Haines et al., 2013; Tamura \& Tanaka, 2014; Wolfson et al., 2015).

\subsection{Study design and assessment of methodological quality}

Appraisal of study quality against 13 criteria (Aromataris \& Munn, 2017) is shown in Table 3. Ten studies were randomized controlled trials, although the method of randomization was unclear in Tamura and Tanaka (2014) and Wolfson et al. (2015) (Table 3, Q1). Tomayko et al. (2018) specified a crossover design but reported postintervention measures from the first pre-crossover period only. Cluster randomization was used in five studies (Bickham et al., 2018; Puder et al., 2011; Tamura \& Tanaka, 2014; Walton et al., 2015; Wolfson et al., 2015), all conducted in schools. Treatment groups were dissimilar at baseline with respect to income, BMI and ethnicity in three studies, all of which used a cluster design (Bickham et al., 2018; Walton et al., 2015; Wolfson et al., 2015), whereas no demographic data besides age and gender was provided in Tamura and Tanaka (2014) (Table 3, Q3). Risk of bias was present among all studies. No studies were able to blind the person delivering the intervention (Table 3, Q5). Blinding of the outcome assessor was reportedly achieved in only three studies (Hammersley et al., 2019; Puder et al., 2011; Walton et al., 2015) (Table 3, Q6) and participants were successfully blinded in just one study (Walton et al., 2015) (Table 3, Q4).

Reporting of the method of allocation concealment (Table 3, Q2) and intentionto-treat versus available-case analysis (Table 3, Q9) was generally inadequate. Outcome measures in all studies (Table 3, Q11) relied on caregiver or participant recall. Questionnaires of unknown reliability and validity were frequently used, with questions such as "On an average weekday how much time per day does your child watch TV?" or "What time does your child usually go to bed on weekdays?" Other measures comprised subsections of the Child Sleep Habits Questionnaire (CSHQ) (Garrison \& Christakis, 2012; Hammersley et al., 2019; Krossbakken et al., 2018), and prospective diaries for sleep (Tamura \& Tanaka, 2014) or screen time (Garrison \& Christakis, 2012). An objective measure of sleep duration (actigraphy) (O’Driscoll, Foster, Davey, Nixon, \& Horne, 2010) was used in one study although compliance issues were reported (Hammersley et al., 2019) (See Appendix VI for details on outcome measures). 
TABLE 3 Assessment of methodological quality of included studies

\begin{tabular}{|c|c|c|c|c|c|c|c|c|c|c|c|c|c|c|}
\hline Study & Q1 & Q2 & Q3 & Q4 & Q5 & Q6 & Q7 & Q8 & Q9 & Q10 & Q11 & Q12 & Q13 & $\begin{array}{l}\% \\
\text { Yes }\end{array}$ \\
\hline $\begin{array}{l}\text { Hammersley } \\
2019^{a}\end{array}$ & $\mathrm{Y}$ & $\mathrm{Y}$ & $\mathrm{Y}$ & $\mathrm{U}$ & $\mathrm{N}$ & $\mathrm{Y}$ & $\mathrm{N}$ & $\mathrm{Y}$ & $\mathrm{Y}$ & $\mathrm{Y}$ & $\mathrm{N}$ & $\mathrm{Y}$ & $\mathrm{Y}$ & $69 \%$ \\
\hline Puder 2011 ${ }^{\mathrm{a}}$ & $\mathrm{Y}$ & $\mathrm{Y}$ & $\mathrm{Y}$ & $\mathrm{U}$ & $\mathrm{N}$ & $\mathrm{Y}$ & $\mathrm{N}$ & $\mathrm{Y}$ & $\mathrm{Y}$ & $\mathrm{Y}$ & $\mathrm{N}$ & $\mathrm{Y}$ & $\mathrm{Y}$ & $69 \%$ \\
\hline $\begin{array}{l}\text { Maddison } \\
2014^{\mathrm{a}}\end{array}$ & $\mathrm{Y}$ & $\mathrm{Y}$ & $\mathrm{Y}$ & $\mathrm{N}$ & $\mathrm{N}$ & $\mathrm{N}$ & $\mathrm{N}$ & $\mathrm{U}$ & $\mathrm{Y}$ & $\mathrm{Y}$ & $\mathrm{N}$ & $\mathrm{Y}$ & $\mathrm{Y}$ & $54 \%$ \\
\hline $\begin{array}{l}\text { Garrison } \\
2012\end{array}$ & $\mathrm{Y}$ & $\mathrm{U}$ & $\mathrm{Y}$ & $\mathrm{U}$ & $\mathrm{N}$ & $\mathrm{U}$ & $\mathrm{Y}$ & $\mathrm{Y}$ & $\mathrm{U}$ & $\mathrm{Y}$ & $\mathrm{N}$ & $\mathrm{Y}$ & $\mathrm{Y}$ & $54 \%$ \\
\hline $\begin{array}{l}\text { Krossbakken } \\
2018\end{array}$ & $\mathrm{Y}$ & $\mathrm{Y}$ & $\mathrm{Y}$ & $\mathrm{N}$ & $\mathrm{N}$ & $\mathrm{U}$ & $\mathrm{U}$ & $\mathrm{Y}$ & $\mathrm{Y}$ & $\mathrm{Y}$ & $\mathrm{N}$ & $\mathrm{Y}$ & $\mathrm{Y}$ & $62 \%$ \\
\hline Haines $2013^{a}$ & $\mathrm{Y}$ & $\mathrm{Y}$ & $\mathrm{Y}$ & $\mathrm{U}$ & $\mathrm{N}$ & $\mathrm{U}$ & $\mathrm{N}$ & $\mathrm{N}$ & $\mathrm{U}$ & $\mathrm{Y}$ & $\mathrm{N}$ & $\mathrm{Y}$ & $\mathrm{Y}$ & $46 \%$ \\
\hline $\begin{array}{l}\text { Tomayko } \\
2018^{\mathrm{a}}\end{array}$ & $\mathrm{Y}$ & $\mathrm{U}$ & $\mathrm{Y}$ & $\mathrm{U}$ & $\mathrm{N}$ & $\mathrm{N}$ & $\mathrm{Y}$ & $\mathrm{Y}$ & $\mathrm{U}$ & $\mathrm{Y}$ & $\mathrm{N}$ & $\mathrm{U}$ & $\mathrm{Y}$ & $46 \%$ \\
\hline Walton $2015^{\mathrm{a}}$ & $\mathrm{Y}$ & $\mathrm{U}$ & $\mathrm{N}$ & $\mathrm{Y}$ & $\mathrm{N}$ & $\mathrm{Y}$ & $\mathrm{Y}$ & $\mathrm{Y}$ & $\mathrm{U}$ & $\mathrm{Y}$ & $\mathrm{N}$ & $\mathrm{U}$ & $\mathrm{U}$ & $46 \%$ \\
\hline $\begin{array}{l}\text { Wolfson } \\
2015^{\mathrm{a}}\end{array}$ & $\mathrm{U}$ & $\mathrm{U}$ & $\mathrm{N}$ & $\mathrm{U}$ & $\mathrm{N}$ & $\mathrm{U}$ & $\mathrm{Y}$ & $\mathrm{Y}$ & $\mathrm{Y}$ & $\mathrm{Y}$ & $\mathrm{N}$ & $\mathrm{U}$ & $\mathrm{U}$ & $31 \%$ \\
\hline Tamura 2014 & $\mathrm{U}$ & $\mathrm{U}$ & $\mathrm{U}$ & $\mathrm{U}$ & $\mathrm{N}$ & $\mathrm{N}$ & $\mathrm{N}$ & $\mathrm{Y}$ & $\mathrm{Y}$ & $\mathrm{Y}$ & $\mathrm{N}$ & $\mathrm{U}$ & $\mathrm{U}$ & $23 \%$ \\
\hline $\begin{array}{l}\text { Bickham } \\
2018^{a}\end{array}$ & $\mathrm{~N}$ & $\mathrm{U}$ & $\mathrm{N}$ & $\mathrm{U}$ & $\mathrm{N}$ & $\mathrm{U}$ & $\mathrm{N}$ & $\mathrm{U}$ & $\mathrm{N}$ & $\mathrm{Y}$ & $\mathrm{N}$ & $\mathrm{U}$ & $\mathrm{U}$ & $8 \%$ \\
\hline$\%$ Yes & $73 \%$ & $45 \%$ & $64 \%$ & $9 \%$ & $0 \%$ & $27 \%$ & $36 \%$ & $73 \%$ & $55 \%$ & $\begin{array}{l}100 \\
\%\end{array}$ & $0 \%$ & $55 \%$ & $64 \%$ & \\
\hline
\end{tabular}

Note: Y, Yes; N, No; U, Unclear

Q1: Was true randomisation used for assignment of participants to treatment groups?

Q2: Was allocation to treatment groups concealed?

Q3: Were treatment groups similar at the baseline?

Q4: Were participants blind to treatment assignment?

Q5: Were those delivering the treatment blind to treatment assignment?

Q6: Were outcomes assessors blind to treatment assignment?

Q7: Were treatment groups treated identically other than the intervention of interest (e.g. attention matched control)?

Q8: Was follow up complete and if not, were differences between groups in terms of their follow up adequately described and analysed?

Q9: Were participants analysed in the groups to which they were randomised? (ITT)

Q10: Were outcomes measured in the same way for treatment groups?

Q11: Were outcomes measured in a reliable way?

Q12: Was appropriate statistical analysis used?

Q13: Was the trial design appropriate and any deviations from the standard RCT design (individual randomisation, parallel groups) accounted for in the conduct and analysis of the trial?

$\%$ Yes: Percentage of studies meeting question criteria

Studies included in meta-analysis ${ }^{a}$ 


\section{5 | Screen time}

Seven studies were included in the pooled analysis of the intervention effect on screen time (hr/day), for a total sample size of 1,904 (Figure 2). A small but statistically significant reduction in screen time of $0.56 \mathrm{hr}(33 \mathrm{~min}) /$ day in favour of the intervention group (95\% CI, 0.20, 0.92), $p=.003$; Figure 2) was indicated. Substantial statistical heterogeneity was present $\left(I^{2}=74 \%\right)$. Sensitivity analyses found that the exclusion of Maddison et al. (2014) reduced attributable variance for screen time from $75 \%$ to $25 \%$ with mean difference (MD) of $-0.37 \mathrm{hr}$ (22 min)/day (95\% CI, $-0.57,-0.17)$.

\subsection{1 | Subgroup analyses}

Table 4 summarizes the results of the subgroup analyses for screen time. No statistically significant differences in treatment effect were noted in subgroups defined by study design (Figure 2), intervention setting or duration. Larger reductions in screen time were observed for interventions with high screen-use focus compared to low screen-use focus; and for interventions delivered face to face compared to indirectly. Intervention effects were stronger in children aged 8-13 years; however, all studies in this subgroup were cluster-randomized/quasiexperimental.

\section{6 | Videogaming and internet use}

Videogaming hours were measured in two studies. Krossbakken et al. (2018) reported no change between treatment groups, whereas Bickham et al. (2018) found an increase in weekday videogaming from baseline of $2 \mathrm{~min} /$ day in the treatment group and $16 \mathrm{~min} /$ day in the control group. Weekend videogaming hours remained unchanged from baseline and between treatment groups (Bickham et al., 2018). Internet use was measured by Bickham et al. (2018) where reductions of $21 \mathrm{~min} / \mathrm{day}$ and $4 \mathrm{~min} /$ day in weekend use were reported for the intervention and control groups, respectively. Weekday internet use reduced only marginally.

\subsubsection{Weekend screen time}

Weekend screen time was reported separately in three studies, with results for weekend internet and videogaming discussed above. Haines et al. (2013) reported 
a reduction in weekend TV viewing of $1.06 \mathrm{hr}(63 \mathrm{~min}) /$ day in the intervention group compared to controls. The intervention group reduced weekend screen time by $0.38 \mathrm{hr}$ (23 $\mathrm{min}) /$ day compared to the control group in Bickham et al. (2018), whereas Hammersley et al. (2019) reported a difference in weekend screen-time hours of $-0.67 \mathrm{hr}$ (40 $\mathrm{min})$ /day between treatment groups, favouring the intervention group.

\subsection{2 | Bedtime screen use}

Refraining from viewing screens in the hour before bedtime was an outcome in two studies. Tamura and Tanaka (2014) found a significant increase in the proportion of children refraining from viewing television or videos before bedtime in the intervention group, from $22.5 \%$ at baseline to $46.5 \%$ of participants postintervention, compared to $25.0 \%-26.3 \%$ in the control. However, the proportion of participants refraining from playing videogames before sleep did not change from baseline. Wolfson et al. (2015) reported a 5-min reduction from baseline in duration of screen use during the hour prior to bedtime in the treatment group, whereas the control group increased bedtime screen use from baseline by 6 min.

\subsection{3 | TV removal from sleeping area}

Removal of the TV from the bedroom was measured in one study. Haines et al. (2013) found no significant change in the proportion of children having had the TV removed from the sleeping area between treatment groups. Haines et al. (2013) reported that $72 \%$ of children aged 2-5 years at baseline were co-sleeping with parents. 


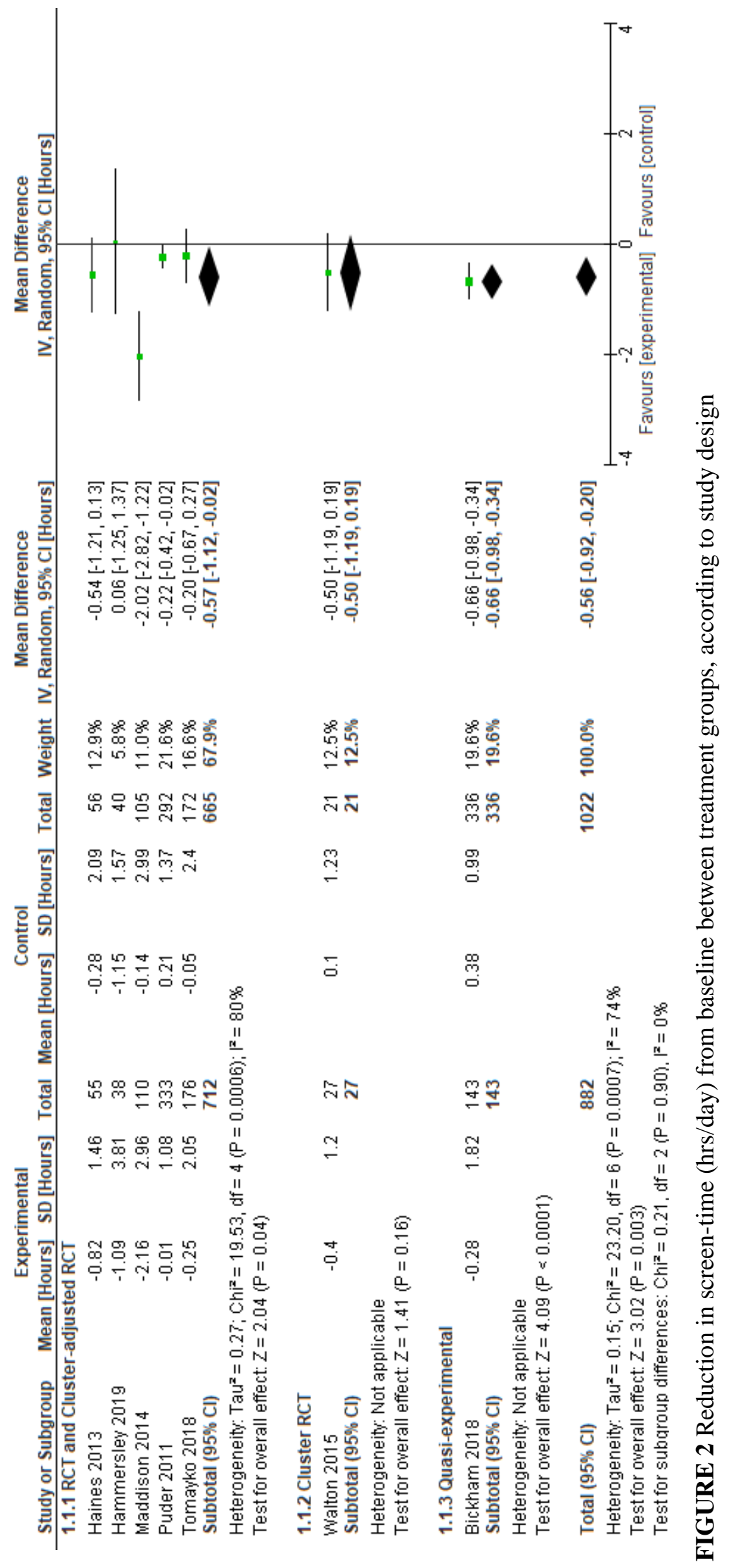


TABLE 4 Subgroup analyses of pooled mean differences in screen time (hr/day) between treatment groups

\begin{tabular}{|c|c|c|c|c|c|}
\hline \multirow[b]{2}{*}{ Subgroup } & \multirow{2}{*}{$\begin{array}{l}\text { No of } \\
\text { studies }\end{array}$} & \multirow{2}{*}{$\begin{array}{l}\text { MD, (hr/day }), \text { random } \\
\text { effects }(95 \% \text { CI })\end{array}$} & \multirow[b]{2}{*}{$p$} & \multicolumn{2}{|c|}{ Heterogeneity } \\
\hline & & & & $x^{2}$ & $I^{2}$ \\
\hline All studies & 7 & $-0.56(-0.92,-0.20)($ Fig. 2) & 0.003 & 23.20 & $74 \%$ \\
\hline \multicolumn{6}{|l|}{ Study design } \\
\hline $\begin{array}{l}\text { RCT \& } \\
\text { Cluster adj. } \\
\text { RCT }\end{array}$ & 5 & $-0.57(-1.12,-0.02)$ & 0.04 & 19.53 & $80 \%$ \\
\hline \multicolumn{6}{|l|}{ Age (years) } \\
\hline $2-6$ & 5 & $-0.25(-0.42,-0.08)$ & 0.004 & 1.57 & $0 \%$ \\
\hline $8-13$ & 2 & $-1.29(-2.62,0.04)$ & 0.06 & 9.69 & $90 \%$ \\
\hline \multicolumn{6}{|l|}{ Intervention } \\
\hline $\begin{array}{l}\text { Setting } \\
\text { School } \\
\text { Home }\end{array}$ & $\begin{array}{l}2 \\
2\end{array}$ & $\begin{array}{l}-0.42(-0.85,0.01) \\
-1.26(-2.71,0.19)\end{array}$ & $\begin{array}{l}0.05 \\
0.09\end{array}$ & $\begin{array}{l}5.38 \\
7.78\end{array}$ & $\begin{array}{l}81 \% \\
87 \%\end{array}$ \\
\hline \multicolumn{6}{|l|}{ Recipient } \\
\hline $\begin{array}{l}\text { Caregiver } \\
\text { Child } \\
\text { Both }\end{array}$ & $\begin{array}{l}2 \\
1 \\
4\end{array}$ & $\begin{array}{l}-0.17(-0.61,0.27) \\
-0.66(-0.98,-0.34) \\
-0.76(-1.46,0.06)\end{array}$ & $\begin{array}{l}0.45 \\
<0.0001 \\
0.03\end{array}$ & $\begin{array}{l}0.13 \\
- \\
19.11\end{array}$ & $\begin{array}{l}0 \% \\
- \\
84 \%\end{array}$ \\
\hline \multicolumn{6}{|l|}{ Duration } \\
\hline $\begin{array}{l}>3 \text { months } \\
<3 \text { months }\end{array}$ & $\begin{array}{l}4 \\
3\end{array}$ & $\begin{array}{l}-0.65(-1.26,-0.05) \\
-0.60(-0.88,-0.32)\end{array}$ & $\begin{array}{l}0.03 \\
<0.0001\end{array}$ & $\begin{array}{l}19.25 \\
1.20\end{array}$ & $\begin{array}{l}84 \% \\
0 \%\end{array}$ \\
\hline \multicolumn{6}{|l|}{ Delivery mode } \\
\hline $\begin{array}{l}\text { Direct } \\
\text { Indirect }\end{array}$ & $\begin{array}{l}5 \\
2\end{array}$ & $\begin{array}{l}-0.70(-1.16,-0.24) \\
-0.17(-0.61,0.27)\end{array}$ & $\begin{array}{l}0.003 \\
0.45\end{array}$ & $\begin{array}{l}21.94 \\
0.13\end{array}$ & $\begin{array}{l}82 \% \\
0 \%\end{array}$ \\
\hline \multicolumn{6}{|c|}{ Aim/focus of intervention } \\
\hline $\begin{array}{l}\text { High ST } \\
\text { Low ST }\end{array}$ & $\begin{array}{l}3 \\
4\end{array}$ & $\begin{array}{l}-1.02(-1.78,-0.25) \\
-0.23(-0.40,-0.06)\end{array}$ & $\begin{array}{l}0.009 \\
0.009\end{array}$ & $\begin{array}{l}10.36 \\
0.80\end{array}$ & $\begin{array}{l}81 \% \\
0 \%\end{array}$ \\
\hline
\end{tabular}

Note: Negative MDs indicate reduction in screen time in the intervention group relative to the control group.

Abbreviations: CI, Confidence interval; High ST, $>1$ session on screen use, or entire program directed at screen-use; Low ST focus, 1 session on screen use; MD, Mean difference; ST, Screen time. 


\section{7 | Sleep duration}

Nine studies were included in the pooled analysis of sleep duration, with a total sample size of 2,164 (Figure 3). A small improvement in sleep duration in favour of the intervention was found, with an MD of $0.19 \mathrm{hr}(11 \mathrm{~min}) / \mathrm{day}$ (95\% CI, 0.05, $0.33 ; p=.008)$ with moderate heterogeneity $\left(I^{2}=43 \%\right)$ (Figure 3$)$. A sensitivity analysis was performed by the sequential removal of single studies, with the pooled MD for sleep duration with one study omitted ranging from $0.15 \mathrm{hr}(9 \mathrm{~min})$ (95\% CI, $\left.0.02,0.29 ; I^{2}=32 \%\right)$ to $0.22 \mathrm{hr}(13 \mathrm{~min}) /$ day $\left(95 \% \mathrm{CI}, 0.06,0.38 ; I^{2}=47 \%\right)$.

\subsection{1 | Subgroup analyses}

When restricted to randomised controlled trial (RCT) designs, the intervention effect was reduced to $0.08 \mathrm{hr}(5 \mathrm{~min}) /$ day $(95 \% \mathrm{CI},-0.07,0.23)$ (Figure 3 ) in favour of the intervention. Results of the subgroup analyses of the pooled mean differences for sleep duration are shown in Table 5. Greater improvement in mean sleep duration hours were observed in children aged 8-13 years compared to children aged 2-6 years, however studies from the older age range were cluster RCTs or quasi-experimental. Although subgroup analysis according to baseline sleep duration was not specified a priori (Martin et al., 2018), when only those studies where participants' average sleep duration at baseline was less than that recommended for age (Hale et al., 2018) were included (Bickham et al., 2018; Hammersley et al., 2015; Tamura \& Tanaka, 2014;), the improvement in sleep duration among the intervention group increased and had less variability $\left(I^{2}=14 \%\right)$. The intervention effect was greater among interventions that were delivered face to face rather than via mail/online; had a high focus on sleep education, and were of less than 3 months' duration. Of the interventions with a high focus on screen time, three reported sleep duration hours (Bickham et al., 2018; Haines et al., 2013; Maddison et al., 2014) with MD of $0.29 \mathrm{hr}$ (17 min)/day.

\subsection{2 | Bedtime, bedtime resistance, sleep latency and sleep problems}

Bedtime was reported by two studies. Tamura and Tanaka (2014) reported that bedtime advanced by $0.28 \mathrm{hr}(17 \mathrm{~min})$ on average in the intervention group, whereas bedtime in the control group remained relatively stable. Wolfson et al. (2015) reported bedtime advancing by an average of $0.16 \mathrm{hr}(10 \mathrm{~min})$ on weeknights, and an hour earlier on weekend nights, for the intervention group relative to controls. 
There was little change in bedtime resistance from baseline between groups (Hammersley et al., 2019; Krossbakken et al., 2018). In Hammersley et al. (2019), a 5-min reduction in sleep latency was detected in the intervention group compared to the controls. The intervention in the study by Garrison and Christakis (2012) was associated with lower odds of "any sleep problem", defined as at least one sleep problem on five to seven nights per week or at least two sleep problems on 2-4 days per week by the caregiver $(\mathrm{OR}=0.36 ; 95 \% \mathrm{CI}, 0.16,0.83)$.

\section{8 | Sustainability of results}

Sustainability of effect was evaluated in three studies, with follow-up periods ranging from 3 to 8 months after intervention completion. Garrison and Christakis (2012) reported a decay in intervention effect over the 6-month period after intervention completion, although the effect was not statistically significant ( $p=$ .7). Hammersley et al. (2019) showed the post-intervention reduction in weekday screen-time was maintained 3 months later, whereas the gain in sleep duration was not sustained. Wolfson et al. (2015) reported no statistically significant change in the intervention effect after 8 months, suggesting the treatment effect was sustained over the follow-up period. 


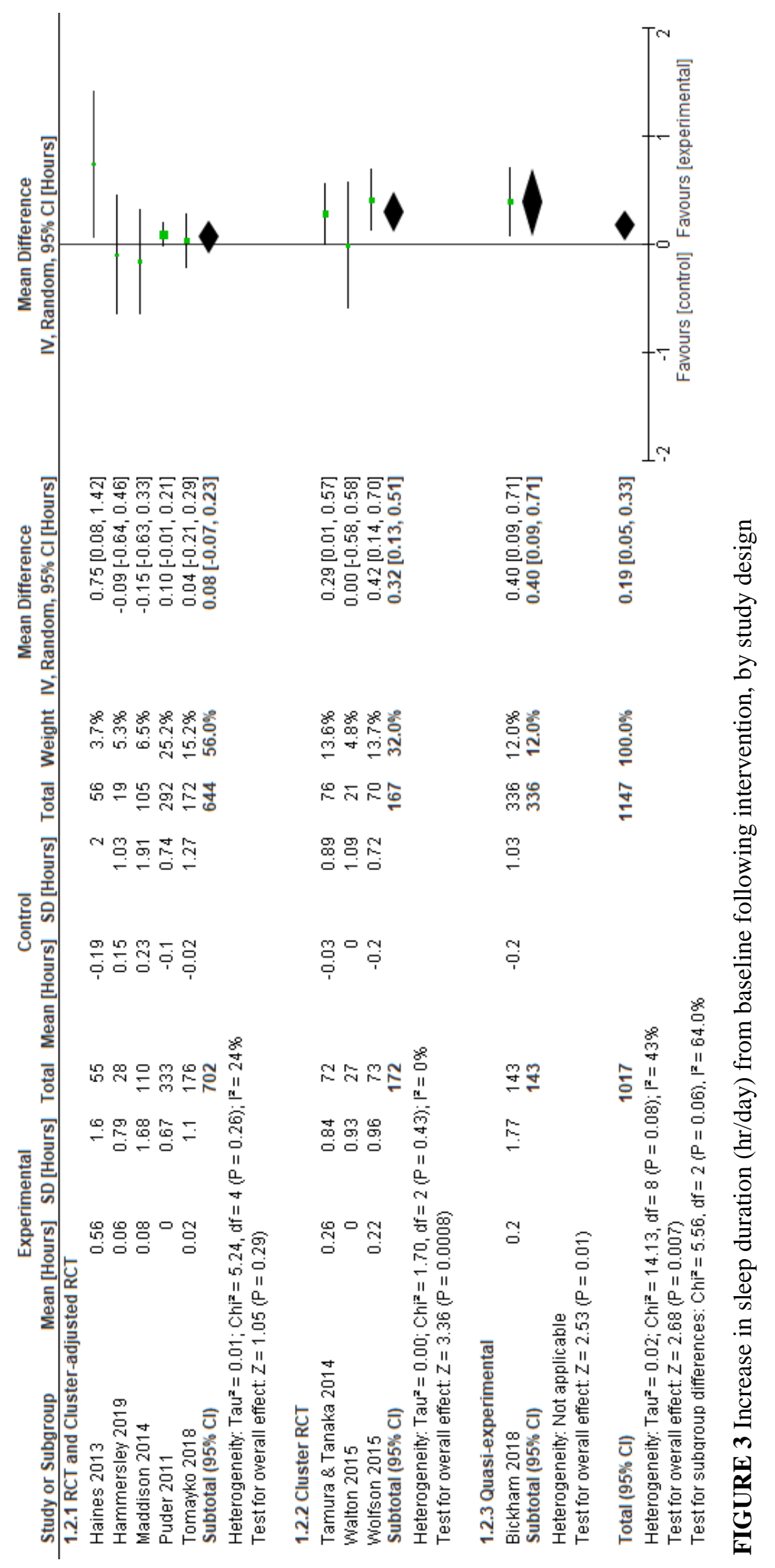

$61 \mid \mathrm{P}$ a g e 
TABLE 5 Subgroup analyses of pooled mean differences in sleep duration (hr/day) between treatment groups

\begin{tabular}{|c|c|c|c|c|c|}
\hline \multirow[b]{2}{*}{ Subgroup } & \multirow{2}{*}{$\begin{array}{l}\text { No of } \\
\text { studies }\end{array}$} & \multirow{2}{*}{$\begin{array}{l}\text { MD, (hr/day }) \\
\text { random effects }(95 \% \mathrm{CI})\end{array}$} & \multirow[b]{2}{*}{$p$} & \multicolumn{2}{|c|}{ Heterogeneity } \\
\hline & & & & $x^{2}$ & $I^{2}$ \\
\hline All studies & 9 & $0.19(0.05,0.33)$ (Fig. 3) & 0.007 & 14.13 & $43 \%$ \\
\hline $\begin{array}{l}\text { Study design } \\
\text { RCT/cluster adj. } \\
\text { RCT }\end{array}$ & 5 & $0.08(-0.07,0.23)$ & 0.29 & 5.24 & $24 \%$ \\
\hline \multicolumn{6}{|l|}{ Age (years) } \\
\hline $\begin{array}{l}2-6 \\
8-13\end{array}$ & $\begin{array}{l}5 \\
4\end{array}$ & $\begin{array}{l}0.09(-0.04,0.22) \\
0.30(0.10,0.49)\end{array}$ & $\begin{array}{l}0.15 \\
0.003\end{array}$ & $\begin{array}{l}4.37 \\
4.45\end{array}$ & $\begin{array}{l}8 \% \\
33 \%\end{array}$ \\
\hline \multicolumn{6}{|l|}{ Intervention } \\
\hline $\begin{array}{l}\text { Setting } \\
\text { School } \\
\text { Home }\end{array}$ & $\begin{array}{l}4 \\
2\end{array}$ & $\begin{array}{l}0.27(0.09,0.45) \\
0.26(-0.62,1.13)\end{array}$ & $\begin{array}{l}0.004 \\
0.57\end{array}$ & $\begin{array}{l}7.39 \\
5.16\end{array}$ & $\begin{array}{l}59 \% \\
81 \%\end{array}$ \\
\hline \multicolumn{6}{|l|}{ Recipient } \\
\hline $\begin{array}{l}\text { Caregiver } \\
\text { Child } \\
\text { Both }\end{array}$ & $\begin{array}{l}2 \\
3 \\
4\end{array}$ & $\begin{array}{l}-0.02(-0.21,0.24) \\
0.37(0.20,0.53) \\
0.11(-0.14,0.37)\end{array}$ & $\begin{array}{l}0.88 \\
<0.0001 \\
0.38\end{array}$ & $\begin{array}{l}0.18 \\
0.48 \\
4.73\end{array}$ & $\begin{array}{l}0 \% \\
0 \% \\
37 \%\end{array}$ \\
\hline \multicolumn{6}{|l|}{ Duration } \\
\hline $\begin{array}{l}>3 \text { months } \\
<3 \text { months }\end{array}$ & $\begin{array}{l}4 \\
5\end{array}$ & $\begin{array}{l}0.09(-0.10,0.27) \\
0.31(0.15,0.46)\end{array}$ & $\begin{array}{l}0.36 \\
0.0001\end{array}$ & $\begin{array}{l}5.35 \\
4.08\end{array}$ & $\begin{array}{l}44 \% \\
2 \%\end{array}$ \\
\hline \multicolumn{6}{|l|}{ Delivery mode } \\
\hline $\begin{array}{l}\text { Direct } \\
\text { Indirect }\end{array}$ & $\begin{array}{l}7 \\
2\end{array}$ & $\begin{array}{l}0.23(0.06,0.40) \\
0.02(-0.21,0.24)\end{array}$ & $\begin{array}{l}0.009 \\
0.88\end{array}$ & $\begin{array}{l}13.26 \\
0.18\end{array}$ & $\begin{array}{l}55 \% \\
0 \%\end{array}$ \\
\hline \multicolumn{6}{|l|}{ Focus } \\
\hline $\begin{array}{l}\text { High ST } \\
\text { High sleep } \\
\text { Low sleep }\end{array}$ & $\begin{array}{l}3 \\
2 \\
7\end{array}$ & $\begin{array}{l}0.29(-0.18,0.76) \\
0.36(0.16,0.55) \\
0.12(-0.04,0.27)\end{array}$ & $\begin{array}{l}0.23 \\
0.0004 \\
0.12\end{array}$ & $\begin{array}{l}7.10 \\
0.42 \\
9.57\end{array}$ & $\begin{array}{l}72 \% \\
0 \% \\
37 \%\end{array}$ \\
\hline \multicolumn{6}{|c|}{ Sleep duration at baseline } \\
\hline $\begin{array}{l}\text { Shorter than } \\
\text { recommended }\end{array}$ & 3 & $0.28(0.06,0.49)$ & 0.01 & 2.33 & $14 \%$ \\
\hline
\end{tabular}

Abbreviations: CI, Confidence interval; High sleep, >1 session on sleep, or entire programme focused on sleep education; Low sleep, 1 session on sleep; MD, Mean difference; ST, screen time; High, $>1$ session on screen use. 


\section{4 | DISCUSSION}

This is the first systematic review to evaluate the effect of interventions for screen use and sleep. Results suggest that such interventions can achieve a small reduction in screen time, a modest improvement in sleep duration, and an earlier bedtime. Estimated reductions in screen time in the present review are consistent with those of previous reviews. Ramsay Buchannan et al.(2016) reported a reduction in screen time among children of $0.44 \mathrm{hr}(26 \mathrm{~min} / \mathrm{day}$; interquartile interval $=0.20,1.24)$ compared to $0.56 \mathrm{hr} /$ day in the present review. Ramsay Buchanan et al. (2016) observed greater screen-time reductions associated with interventions that specifically targeted screen time, compared to those targeting multiple lifestyle behaviours. This finding was supported in the current review, where subgroup analyses indicated larger effects when multi-component studies, most with a primary objective of reducing BMI, were excluded. Interventions targeting multiple lifestyle behaviours tended to be of longer duration, and any behaviour changes achieved from the one or two sessions addressing sleep or screen time may not be consolidated as further topics are introduced. Similarly, in a systematic review by Wu et al. (2016) the MD for screen-time reduction was strengthened when only single-focus interventions were considered. The mean reduction was also larger when analysis was restricted to interventions of less than 7 months duration. In the current review, effects of interventions on sleep duration, but not screen time, were larger when only shorter duration studies were included.

Friedrich et al. (2014) reported a reduction in screen time of $0.25 \mathrm{hr}(15 \mathrm{~min}) /$ day (95\% CI, 0.13, 0.37) for screen-time reduction interventions conducted in the school setting. In the present review, the reduction in screen time for such interventions was more pronounced ( $0.44 \mathrm{hr}$ (26 $\mathrm{min}) /$ day), and slightly less than the overall effect (-0.56 hr (34 min)/day).

A narrative synthesis by Schmidt et al. (2012) suggested the intervention setting was of little consequence provided programmes had high levels of family involvement, a finding also supported in a review by Marsh et al. (2014). Results in the present review did not significantly alter according to intervention setting, whereas effects of interventions delivered 'face to face' with the child and/or caregiver were greater relative to those delivered online or indirectly. 
In a review by Wahi et al. (2011), overall reductions in screen time were only observed in subgroup analysis for age, yielding a reduction of $3.72 \mathrm{hr} /$ week $(0.53$ $\mathrm{hr}(31 \mathrm{~min}) /$ day $)(95 \% \mathrm{CI}, 0.20,7.23)$ in children aged under 6 years. This is considerably more than the $0.25 \mathrm{hr}(15 \mathrm{~min}) /$ day reduction for this age group in the current review, where greater reductions were observed among older children. Similarly, Maniccia et al. (2011) found the magnitude of intervention effect was greater among children aged over 5 years, compared to children aged under 5 years. Although such inconsistency may be attributable to the high heterogeneity present in interventions conducted for screen-time reduction, further consideration of intervention design with respect to participant age is warranted.

The gains in sleep duration associated with the interventions reviewed were minimal (11-18 min/day). Among interventions specifically focusing on sleep, the average effect was slightly greater ( $21 \mathrm{~min} /$ day). These findings are consistent with Yoong et al. (2016), who reported little impact of multicomponent interventions aiming to improve sleep and BMI. In a meta-analysis of cognitive behavioral interventions targeting sleep in adolescents, Blake, Sheeber, Youssef, Ranti, and Allen (2017) reported an average improvement of $0.49 \mathrm{hr}$ (29 min) sleep/day (95\% CI, 17.18, 41.75). This suggests effects may be larger when sleep is the primary intervention focus. Other systematic reviews assessing the effectiveness of sleep interventions in children reported on outcomes other than sleep duration, such as sleep latency and night wakings (Meltzer \& Mindell, 2014).

Children's sleep requirements vary according to age. Based on the reported mean sleep duration at baseline, it is likely that at least some of the participants in the included studies were achieving adequate sleep prior to the intervention. The potential improvement in sleep duration among these participants may be limited, which may bias intervention effects toward zero. Future interventions may consider targeting those at risk of developing poor health outcomes, including obesity, or those already demonstrating excessive levels of screen use and/or inadequate sleep. Similarly, randomization stratified according to baseline BMI, screen-use hours and/or sleep duration should be considered. Interventions aiming to control screen use may benefit by addressing and measuring, at baseline and post-intervention, additional factors associated with excessive screen use. These include parental selfefficacy in controlling their child's screen use (Goncalves, Byrne, Tavares, Viana, 
\& Trost, 2019), household chaos (Emond et al., 2018), parental use of electronic media (Emond et al., 2018; Goncalves et al., 2019) and the presence of electronic media in sleeping environments (Jago et al., 2013).

Few of the included studies followed-up participants after completion of the intervention, such that sustainability of intervention effect(s) and the long-term acceptability and maintenance of strategies employed could not be determined. This issue was raised in previous reviews, particularly in regards to acceptability of TV control devices and contingency feedback systems (Maniccia et al., 2011; Wu et al., 2016). There appears to be poor compliance and low acceptability with respect to modification of the sleeping environment (such as by removing the TV from the room in which the child sleeps), which may limit its long-term effectiveness (Maniccia et al., 2011). It may be that caregivers who co-sleep with younger children, which is common (Haines et al., 2013), may be reluctant to remove the TV from their own bedroom. Many studies used incentives such as gift cards to increase compliance and encourage attendance at follow-up assessments, making it difficult to ascertain the true acceptability of the intervention and likelihood of maintenance of behaviour changes in the long term.

There is a need for studies to utilize objective measures of screen time and sleep, such as prospective time-use diaries, screen-time/phone-use tracker applications and actigraphy via smart phones and watches. Efforts should be made to accurately measure total screen time across the range of different screen-based media devices, as reduction in time spent using one device may be offset by increased time using an alternate device. Also, although TV may have been the preferred screen type among young children in the past, this may have shifted owing to the increasing popularity of tablets and smartphones among this demographic (Rideout, 2017). More RCTs evaluating interventions whose primary focus is daytime and evening screen use in children and their effects on sleep behaviour are needed. Increased intervention specificity may also reduce the statistical heterogeneity observed and provide more precise estimates of intervention effect. The screen-use interventions included in this review appeared to have little effect on videogaming behaviour. This may suggest that the factors driving videogaming in children differ to those driving other screen-based behaviours, and interventions aiming to address videogaming may require greater specificity than those evaluated in this review. 


\section{1 | Limitations}

The present review has limitations. Study selection and data extraction were completed by one person, increasing the risk that potentially eligible studies were missed (Higgins \& Green, 2011). Of the small number of eligible studies identified, most had small sample sizes, utilized different methodologies and inadequately controlled for potential sources of bias. Cluster RCTs are common in research conducted in the school setting, particularly when the intervention is embedded within the school curriculum. However, such studies must ensure cluster number and size are adequate, and that appropriate statistical methods are applied in the analysis. If the unit of randomization is "school", an adequate number of schools must be recruited, rather than simply allocating or "randomizing" one school to the treatment group and another school to the control group, as was done in Bickham et al. (2018) and Wolfson et al. (2015). Cluster formation and composition were inadequately described in two papers (Tamura \& Tanaka, 2014; Walton et al., 2015) such that adjustments needed to account for clustering could not be applied. The considerable variability among the included interventions may explain some of the statistical heterogeneity observed. Minimal confidence can be placed in the results from the cluster RCTs/quasi-experimental studies, most of which were conducted in schools, which has implications for the subgroup analyses for age and setting. Screen time and sleep were mostly secondary outcomes and were therefore likely to be underpowered to detect differences in these outcomes. Most measures of screen time and sleep relied on retrospective recall by the caregiver or child, and is susceptible to several types of bias, including recall bias and social desirability. A further limitation was the need to calculate the variance for mean change from baseline where it was not reported. This involved applying a range of correlation coefficients, which had to be estimated. Use of such approximations would not be necessary if studies reported more detailed results and utilized appropriate statistical methods and objective outcome measures, which would in turn provide greater certainty of results.

\section{5 | CONCLUSIONS}

Results of the present systematic review and meta-analysis suggest interventions aiming to reduce screen time and improve sleep duration may be effective in 
achieving modest reductions in screen time, increases in sleep duration and an earlier bedtime in children, but were largely ineffective in altering videogaming behaviour. Whether controlling screen use alone can result in improved sleep is yet to be conclusively determined, as many of the included studies comprised cointerventions and studies evaluating interventions specifically targeting screen use and sleep were of limited number and of varying methodological quality.

\section{ACKNOWLEDGEMENTS}

Dr Kylie Porritt assisted in the development of the review. Judith Lowe performed the second appraisal of study design quality. Maureen Bell assisted in developing the initial search strategy.

\section{CONFLICT OF INTERESTS}

All the authors have no conflict of interests to disclose.

\section{AUTHOR CONTRIBUTIONS}

$\mathrm{KBM}$ and ECA conceptualized and designed the study. KBM performed the search and technical aspects, including study appraisal, extraction, and data analysis. ECA confirmed technical details, including study inclusion and presentation of results. JMB contributed to conduct of statistical analyses and presentation of results. KBM drafted the initial manuscript. KBM, JMB and ECA contributed to multiple drafts of the manuscript. All authors approved the final manuscript as submitted and agree to be accountable for all aspects of the work.

\section{REFERENCES}

Akacem, L. (2016). Understanding the circadian physiology in early childhood: The role of napping and light at night. Integrative Physiology Graduate Theses and Dissertations. 51.

Aromataris, E., \& Munn, Z. (Eds.) (2017). Joanna Briggs Institute reviewer's manual. Adelaide, SA, The Joanna Briggs Institute. Retrieved from https://reviewersmanual.joannabriggs.org/

Bickham, D., Hswen, Y., Slaby, R., \& Rich, M. (2018). A preliminary evaluation of a school-based media education and reduction intervention. Journal of Primary Prevention, 39, 229-245. https://doi.org/10.1007/s10935-018-0510-2

Blake, M., Sheeber, L., Youssef, G., Raniti, M., \& Allen, N. (2017). Systematic review and meta-analysis of adolescent cognitive-behavioral sleep interventions. Clinical Child and Family Psychology Review, 20(3), 227-249. https://doi.org/10.1007/s10567-017$0234-5$

Cain, N., \& Gradisar, M. (2010). Electronic media use and sleep in school-aged children and adolescents: A review. Sleep Medicine, 11, 735-742. https://doi.org/j.sleep.2010.02.006

Carter, B., Rees, P., Hale, L., Bhattacharjee, D., \& Paradkar, M. (2016). Association between portable screen-based media device access or use and sleep outcomes: A 
systematic review and meta-analysis. JAMA Pediatrics, 170(12), 1202-1208. http://doi.org/10.1001/jamapediatrics.2016.2341

Cespedes, E.M., Rifas-Shiman, S.L., Redline, S., Gillman, M.W., Peña, M.M., \& Taveras, E.M. (2014). Longitudinal associations of sleep curtailment with metabolic risk in midchildhood. Obesity, 22(12), 2586-2592.

Dube, N., Khan, K., Loehr, S., Chu, Y., \& Veugelers, P. (2017). The use of entertainment and communication technologies before sleep could affect sleep and weight status: a population-based study among children. International Journal of Behavioral Nutrition and Physical Activity, 14, 97. https://doi: 10.1186/s12966-017-0547-2

Emond, J., Tantum, L., Gilbert-Diamond, D., Kim, S., Lansigan, R., \& Neelon, S. (2018). Household chaos and screen media use among pre-school-aged children: a crosssectional study. BMC Public Health, 18, 1210. https://doi.org/10.1186/s12889-018$6113-2$

Friedrich, R. R., Polet, J. P., Schuch, I., \& Wagner, M. B. (2014). Effect of intervention programs in schools to reduce screen time: A meta-analysis. Journal De Pediatria, 90, 232-241. https://doi.org/10.1016/j.jped.2014.01.003

Garmy, P., Clausson, E., Nyberg, P., \& Jakobsson, U. (2018). Insufficient sleep is associated with obesity and excessive screen time amongst ten-year-old children in Sweden. Journal of Pediatric Nursing, 39, e1-e5.

Garrison, M. M., \& Christakis, D. A. (2012). The Impact of a healthy media use intervention on sleep in preschool children. Pediatrics, 130(3), 492-499. https://doi.org/10.1542/peds.2011-3153

Garrison, M., Liekweg, K., \& Christakis, D. (2011). Media use and child sleep: the impact of content, timing and environment. Pediatrics, 128(1). https://doi.org/10.1542/peds.2010-3304

Goncalves, W., Byrne, R., Tavares, E., Viana, M., \& Trost, S. (2019). Parental influences on screen time and weight status among preschool children from Brazil: A crosssectional study. International Journal of Behavioral Nutrition and Physical Activity, 16, 27. https://doi.org/10.1186/s12966-019-0788-3

Haines, J., McDonald, J., O’Brien, A., Sherry, B., Bottino, C.J., Schmidt, M.E., \& Taveras, E. (2013). Healthy Habits, Happy Homes: Randomized trial to improve household routines for obesity prevention among preschool-aged children. JAMA Pediatr, 167(11), 1072-1079.

Hale, L., and Guan, S. (2015). Screen time and sleep among school-aged children and adolescents: A systematic literature review. Sleep Medicine Reviews, 21, 50-8.

Hale, L., Kirschen, G., LeBourgeois, M., Gradisar, M., Garrison, M., MontgomeryDowns, H., ... Buxton, O. (2018). Youth screen media habits and sleep, sleepfriendly screen behaviour recommendations for clinicians, educators and parents. Child and Adolescent Psychiatric Clinics of North America, 27, 220-245.

Hammersley, M., Okely, A., Batterham, M., \& Jones, R. (2019). An internet-based childhood obesity prevention program (Time2bHealthy) for parents of preschool-aged children: Randomized controlled trial. Journal of Medical Internet Research, 21(2), e11964. https://doi.org/10.2196/11964

Harris, K.C., Kuramoto, L.K., Schulzer, M., \& Retallack, J.E. (2009). Effect of schoolbased physical activity interventions on body mass index in children: A meta-analysis. 
Canadian Medical Association Journal, 180, 719-729. https://doi.org/10.1503/cmaj.080966

Higgins, J. P. T., \& Green, S. (2011). Cochrane handbook for systematic reviews of interventions, version 5.1.0 (updated March 2011). The Cochrane collaboration. Retrieved from: http://www.cochrane-handbook.org

Hiscock, H., Canterford, L., Ukoumunne, O. C., \& Wake, M. (2007). Adverse associations of sleep problems in Australian preschoolers: National Population Study. Pediatrics, 119(1), 86-93. https://doi.org/10.1542/peds.2006-1757

Jago, R., Sebire, S., Lucas, P., Turner, K., Bentley, G., Goodrend, J., ... Fox, K. (2013). Parental modelling, media equipment and screen-viewing among children: Crosssectional study. British Medical Journal Open, 3, e002593. https://doi.org/10.1136/bmjopen-2013-002593

Krossbakken, E., Torsheim, T., Mentzoni, R., King, D., Bjorvatn, B., Lorvic, I., \& Pallesen, S. (2018). The effectiveness of a parental guide for prevention of problematic video gaming in children: A public health randomized controlled intervention study. Journal of Behavioral Addictions, 7(1), 52-61. https://doi.org/10.1556/2006.6.2017.087

Lange, K., Cohrs, S., Skarupke, C., Gorke, M., Szagun, B., \& Schlack, R. (2017). Electronic media use and insomnia complaints in German adolescents: Gender differences in use patterns and sleep problems. Journal of Neural Transmission, 124, 79-87. https://doi.org/10.1007/s00702-015-1482-5

Maddison, R., Marsh, S., Foley, L., Epstein, L., Olds, T., Dewes, O., ... Mhurchu, C. (2014). Screen-time weight loss intervention targeting children at home (SWITCH): A randomised controlled trial. Journal of Behavioral Nutrition and Physical Activity, 11, 111.

Maniccia, D. M., Davison, K. K., Marshall, S. J., Manganello, J. A., \& Dennison, B. A. (2011). A meta-analysis of interventions that target children's screen time for reduction. Pediatrics, 128(1), e193-207. https://doi.org/10.1542/peds.2010-2353

Marsh, S., Foley, L. S., Wilks, D. C., \& Maddison, R. (2014). Family-based interventions for reducing sedentary time in youth: A systematic review of randomized controlled trials. Obesity Reviews, 15, 117-133. https://doi.org/10.1111/obr.12105

Martin, K., Porritt, K., \& Aromataris, E. (2018). Effectiveness of interventions to control screen use and children's sleep, cognitive and behavioral outcomes: A systematic review protocol. JBI Database of Systematic Reviews and Implementation Reports, 16(6), 1338-1345.

Masur, E. F., Flynn, V., \& Olson, J. (2015). The presence of background television during young children's play in American homes. Journal of Children Media, 9(3), 349-367.

Meltzer, L., \& Mindell, J. A. (2014). Systematic review and meta-analysis of behavioral interventions for pediatric insomnia. Journal of Pediatric Psychology, 39(8), 932-948. https://doi.org/10.1093/jpepsy/jsu041

Moher, D., Liberati, A., Tetzlaff, J., \& Altman, D.G. (2009). PRISMA Group. Preferred reporting items for systematic reviews and meta-analysis: The PRISMA statement. $B M J, 339, \mathrm{~b} 2535$.

National Sleep Foundation (2014). Sleep in America Poll: Sleep in the modern family. Washington, DC: The Foundation. Retrieved from http://www.sleepfoundation.org/sleep-polls-data/sleep-in-americapoll/2014-sleep-inthe-modern-family. National Sleep Foundation 2014 Sleep America Poll. 
O’Driscoll, D. M., Foster, A. M., Davey, M. J., Nixon, G. M., \& Horne, R. S. C. (2010). Can actigraphy measure sleep fragmentation in children? Archives of Disease in Childhood, 95(12), 1031-1033. https://doi.org/10.1136/abc.2009.166561

Puder, J., Marques-Vidal, P., Schindler, C., Zahner, L., Niederer, I., Burgi, F., ... Kriemler, S. (2011). Effect of a multidimensional lifestyle intervention on fitness and adiposity in predominantly migrant preschool children (Ballabeina): Cluster randomised controlled trial. BMJ, 343, d6195. https://doi.org/10.1136/bmj.d6195.

Ramsey Buchanan, L., Rooks-Peck, C. R., Finnie, R. K., Wethington, H. R., Jacob, V., Fulton J. E., ... Glanz, K. (2016). Reducing recreational sedentary screen time: A community guide systematic review. American Journal of Preventive Medicine, 50(3), 402-415. https://doi.org/10.1016/j.amepre.2015.09.030

Rideout, V. (2017). The common sense census: Media use by kids age zero to eight. San Francisco, CA: Common Sense Media.

Sadeh, A., Mindell, J. A., Luedtke, K., \& Wiegand, B. (2009). Sleep and sleep ecology in the first 3 years: A web-based study. Journal of Sleep Research, 18, 60-73. https://doi.org/10.1111/j.1365-2869.2008.00699.x

Schmidt, M. E., Haines, J., O’Brien, A., McDonald, J., Price, S., Sherry, B., \& Taveras, E. M. (2012). Systematic review of effective strategies for reducing screen time among young children. Obesity, 20(7), 1338-1354. https://doi.org/10.1038/oby.2011.348

Steeves, J., Thompson, D., Bassett, D., Fitzhugh, E., \& Raynor, H. (2011). A review of different behavior modification strategies designed to reduce sedentary screen behaviors in children. Journal of Obesity, 2012, 379215. https://doi.org/10.1155/2012/279215

Tamura, N., \& Tanaka, H. (2014). Effects of sleep education with self-help treatment for elementary schoolchild with nocturnal lifestyle and irritability. Sleep and Biological Rhythms, 12, 169-179. https://doi.org/10.1111/sbr.12055

Tomayko, E. J., Prince, R. J., Cronin, K. A., Kim, K., Parker, T., \& Adams, A. K. (2019). The healthy children, strong families 2 (HCSF2) randomized controlled trial improved healthy behaviors in American Indian families with young children. Current Developments in Nutrition, 3(Suppl 2), 53-62.

Wahi, G., Parkin, P. C., Beyene, J., Uleryk, E. M., \& Birken, C. S. (2011). Effectiveness of interventions aimed at reducing screen time in children: A systematic review and meta-analysis of randomized controlled trials. Archives of Pediatrics and Adolescent Medicine, 165(11), 979-986. https://doi.org/10.1001/archpediatrics.2011.122

Walton, K., Filion, J., Gross, D., Morrongiello, B., Darlington, G., Simpson, J., ... Haines, J. (2015). Parents and tots together: Pilot randomized controlled trial of a family based obesity prevention intervention in Canada. Canadian Journal of Public Health, 106(8), e555-e562. https://doi.org/10.1097/MD.0000000000004029

Wolfson, A., Harkins, E., Johnson, M., \& Marco, C. (2015). Effects of the young adolescent sleep smart program on sleep hygiene practices, sleep health efficacy, and behavioral well-being. Sleep Health, 1, 197-204. https://doi.org/10.1016/j.sleh.2015.07.002

Wu, L., Sun, S., He, Y., \& Jiang, B. (2016). The effect of interventions targeting screen time reduction: A systematic review and meta-analysis. Medicine, 95(27), e4029. https://doi.org/10.1097/MD.0000000000004029

Yland, J., Guan, S., Emanuele, E. \& Hale, L. (2015). Interactive vs passive screen time and nighttime sleep duration among school-aged children. Sleep Health, 191-196. https://doi.org/10.1002/oby.21459 
Yoong, S. L., Chai, L. K., Williams, C. M., Wiggers, J., Finch, M., \& Wolfenden, L. (2016). Systematic review and meta-analysis of interventions targeting sleep and their impact on child body mass index, diet, and physical activity. Obesity, 24(5), 1140-1147. https://doi.org/10.1002/oby.21459

\section{AUTHOR BIOGRAPHIES}

Katie B. Martin, Masters of Clinical Science Candidate (Higher degree by research) with the Joanna Briggs Institute, The University of Adelaide, South Australia. Masters Degree in Occupational Therapy, and a Bachelor of Applied Science in Human Movement, with the University of South Australia.

Jana M. Bednarz, Statistician, Adelaide Health Technology Assessment, Adelaide Health and Medical Sciences, The University of Adelaide.

Edoardo C. Aromataris, Associate Professor, Director Synthesis Science, Joanna Briggs Institute, Faculty of Health and Medical Sciences, The University of Adelaide. 


\section{Chapter 4 Discussion and conclusions}

This thesis presents the first systematic review evaluating the effect of interventions to control children's screen use and their influence on screen use and sleep. The following chapter will expand on the discussion presented in chapter 3, particularly implications for the review findings considering the multitude of variables that influence screen use and sleep in children.

The results of the present review indicate that interventions targeting screen use in children can achieve a small but significant reduction in screen time and improvement in sleep. The estimated screen-time reductions are consistent with those reported by previous reviews comparing similar interventions, in which reductions in screen-time ranged from 8 minutes $^{11}$ to 39 minutes ${ }^{17}$ per day. The gains in sleep duration associated with the interventions reviewed were minimal (11 to 18 minutes per day). Interestingly, of the interventions with a high focus on screen time (defined as including more than one education session on screen use) and with minimal focus on sleep, (defined as one or no education sessions on sleep) three studies reported sleep duration hours ${ }^{84-86}$ with a mean difference of 17 minutes per day. This is comparable to the gain in sleep duration observed in the included studies that specifically focused on sleep ${ }^{87,88}$ and indicates the importance of further research on measuring the effect a reduction in screen use has on sleep, in support of establishing the relationship between screen use and poor sleep. Few systematic reviews assessing the effectiveness of sleep interventions in children exist and report on outcomes other than sleep duration, such as sleep latency and night wakings ${ }^{89}$ making comparison difficult.

\subsection{Influence of intervention parameters on outcomes}

The intervention effect was observed to be altered when subgroup analysis for varying intervention characteristics were completed, such as intervention focus, duration and delivery. 


\subsubsection{Intervention 'focus'}

When considering intervention specificity or 'focus', differences in intervention effect were observed. Subgroup analyses indicated larger effects when multicomponent studies, most with a primary objective of reducing BMI, were excluded. The interrelationship between obesity, screen time and sleep duration may warrant the implementation of such interventions targeting multiple health-related behaviours for change, such as diet, physical activity, screen time and sleep and may be effective in reducing BMI. However, the effects of such interventions on reducing screen time and improving sleep outcomes were less pronounced compared to interventions that specifically targeted either screen use or sleep. This was also observed in a review by Ramsay Buchanan et al. ${ }^{13}$ reporting greater screen-time reductions with interventions that specifically targeted screen time, compared to those targeting multiple lifestyle behaviours. Interventions targeting multiple lifestyle behaviours tended to be of longer duration, and any behaviour changes achieved from the one to two sessions addressing sleep or screen time may not be consolidated as further topics are introduced. ${ }^{13,17}$ Similarly, in a systematic review by $\mathrm{Wu}$ et $a l .{ }^{17}$ the mean difference for screen-time reduction was strengthened when only single-focus interventions were considered.

For studies that provided separate results for screen type, narrative analysis was necessary due to limited comparable data. While most interventions reported some reduction in screen time for at least one screen type, the studies that measured videogaming hours or videogaming behaviour ${ }^{86,87,90}$ were unable to elicit a desired change in behaviour. These studies evaluated different types of interventions, namely a hard-copy guide to preventing problematic videogaming behaviour, ${ }^{90} \mathrm{a}$ sleep hygiene education program ${ }^{87}$ and a screen-use education program. ${ }^{86}$ Although the latter two interventions were able to achieve a reduction in screen time ${ }^{86}$ and screen-viewing habits, ${ }^{87}$ videogaming behaviour remained unchanged. This may suggest that interventions aiming to address videogaming behaviour require highly tailored strategies to create changes in this behaviour. Children displaying problematic videogaming behaviour may meet the criteria for 'internet gaming disorder' (IGD), an area that has limited research into the prevalence of this condition among children, though a larger number of studies have investigated the occurrence of IGD among adolescents. ${ }^{91}$ Estimates indicate IGD may affect, on 
average, $2 \%$ of children and adolescents from representative samples and 5.5\% when including clinical samples. ${ }^{91}$ A common theme among studies and reviews on IGD in youth is the role of family interactions and parenting skills in the progression from non-problematic videogaming to IGD, and also exposure and access to violent videogames. ${ }^{91,92}$ The studies evaluating videogaming included in this thesis had either minimal or no caregiver involvement, and did not provide any support or counselling to improve parental skill in managing problematic videogaming behaviour. Interventions for problematic videogaming and IGD need to have therapeutic methods in place to address any underlying relationship issues within the family and should incorporate family therapy and cognitive behaviour therapy as well as address social, emotional, cognitive and behavioural variables in the intervention. ${ }^{93}$

Among interventions specifically focusing on sleep, the average effect was slightly greater, with sleep duration extended by 21 minutes compared 11 to 18 minutes for interventions with less content focusing on sleep. These findings are consistent with Yoong et al. ${ }^{94}$ and are not dissimilar to that found by Blake et al. ${ }^{95}$ with gains in sleep duration of 29 minutes. This suggests effects may be larger when sleep is the primary or only intervention focus; however, while sleep may be the sole focus of an intervention, it need not be the only outcome of interest. Including a range of outcomes that may potentially be altered by improving sleep alone, such as body weight, dietary intake, academic performance and behaviour, for example, would provide greater insight into the significance of sleep, as well as other influencing factors.

\subsubsection{Intervention content and specific strategies}

Differences in intervention effect have been found in relation to intervention content delivery and the type of intervention strategies employed to achieve the desired behaviour change. In the review by $\mathrm{Wu}$ et al., ${ }^{17}$ intervention effect was strengthened in subgroup analysis of studies that included counselling and education as the primary means of reducing screen time. While subgroup analysis according to counselling and education was not performed in the current review, subgroup analysis for interventions delivered face-to-face with the child and/or 
caregiver (all of which included counselling and education) were greater relative to those delivered online or indirectly.

\section{a. Television monitoring devices}

Another frequently employed strategy to reduce screen time is the use of television monitoring devices, evaluated in one study in the present review. ${ }^{85}$ The effectiveness of television monitoring devices in reducing screen time has been previously reviewed and been shown to be effective in reducing screen time, however, with low acceptability. In contrast, $\mathrm{Wu}$ et $a l .{ }^{17}$ found the use of television monitoring devices to be less effective in reducing or measuring screen time, proposing television viewing may have been replaced by viewing other screenbased media. Issues with the use and acceptability of television-control devices was evident in Maddison et al., ${ }^{85}$ where $46 \%$ of the participants reportedly chose not to use the 'Time Machine' component of the intervention to budget media use.

\section{b. Removal of the television from the bedroom}

One frequently cited risk factor for excessive screen time, sleep and obesity, is the presence of electronic media in the sleeping environment, ${ }^{96}$ as discussed in chapter 1. Most studies included in the present review recorded the presence of a television in the bedroom of participants in addition to other screen-use measures, and removal of the television from the bedroom was often a recommendation of the intervention under evaluation; however, only one study included this as an outcome measure. ${ }^{84}$ At baseline, Haines et al ${ }^{84}$ found that $100 \%$ of the participants had a television in the bedroom, with a $20 \%$ reduction post intervention within the intervention group. A $12 \%$ reduction was observed in the control group, with the between group intervention effect non-significant. This is a greater reduction in the presence of a television in the bedroom than that reported by a large multi-country, pre-test post-test control-group study aiming to address obesity and sleep in children aged two to nine years, excluded from this review (Appendix V). ${ }^{50}$ The aforementioned study found a small increase in the amount of participants from both treatment groups (though a smaller increase among the intervention group compared to control) with a television in the bedroom over the two-year intervention period. ${ }^{50}$ Given the correlation between bedtime screen use, total 
screen time, obesity and sleep insufficiency with the presence of a television or electronic media in the bedroom, further research specifically targeting the physical sleep environment is needed. The apparent increase in the presence of a bedroom television set as a child age ${ }^{22,50}$ highlights the importance of intervening at an early age to prevent this trend in behaviour.

\section{c. Restricting bedtime screen use}

Restricting screen use in the hour prior to and at bedtime was a practice recommended in most included studies, and was an outcome measure reported in two studies included in the present review, ${ }^{85,88}$ both yielding reductions in bedtime screen use; however, no change to videogaming prior to bedtime was achieved. General recommendations suggest limiting screen use in the hour prior to set bedtime in an effort to reduce the negative effect screen use may have on sleep; however, little evidence to support the one-hour duration of screen-free time prior to bedtime exists. It may be that limiting screen use for one hour prior to bedtime is insufficient to negate the potential ill-effects of screen use on sleep. It may also depend on the amount of screen time occurring prior to the screen-free period prior to bedtime. Further research investigating how varying the duration of screen-free time prior to bedtime alters sleep outcomes is required. Having a better understanding of how the length of screen-free time prior to bedtime effects sleep would provide greater clarity in setting recommendations for limiting bedtime screen use.

\subsubsection{Intervention duration and setting}

Another factor that may influence intervention effect is intervention duration. When analysis was restricted to interventions of less than seven months' duration, the mean reduction in screen time reported by $\mathrm{Wu}$ et $a l .{ }^{17}$ was larger when compared to interventions of longer duration, which was partially supported in the current review as the effect of interventions on sleep duration, but not screen time, were larger when only shorter-duration studies were included.

Based on previous reviews, the effect of the setting in which the study is implemented may have negligible influence on results or be less important than other intervention characteristics. According to Friedrich et al., ${ }^{10}$ a reduction in 
screen time of 15 minutes per day $(95 \% \mathrm{CI}, 0.13,0.37)$ after screen-time reduction interventions were conducted in the school setting was observed. In the present review, the reduction in screen time for such interventions was more pronounced with 26 minutes per day, and slightly less than the overall effect of 34 minutes per day. A narrative synthesis by Schmidt et al. ${ }^{14}$ suggested intervention setting was of little consequence provided programs had high levels of family involvement, a finding also supported in a review by Marsh et al. ${ }^{12}$ Similarly, results in the present review did not significantly alter according to intervention setting.

\subsection{Intervention effect according to participant variables}

\subsubsection{Participant age}

Intervention effect has been observed to be either strengthened or reduced when considering participant age. In a review by Wahi et al., ${ }^{16}$ overall reductions in screen time were only observed in subgroup analysis for age, yielding a reduction of 31 minutes per day in children aged under six years. This is considerably more than the 15 minutes per day reduction for this age group in the current review, where greater reductions were observed among older children. Similarly, Maniccia et al. ${ }^{11}$ found the magnitude of intervention effect was greater among children aged over five years, compared to children aged under five years. While such inconsistency may be attributable to the high heterogeneity present in interventions conducted for screen-time reduction, further consideration of intervention design with respect to participant age is warranted. This is particularly important given the age-related differences in viewing habits, screen type preferences, and the nature of the content viewed, as presented in chapter 1 .

\subsubsection{Gender}

Differences exist in the screen-viewing habits and preferences of adolescent boys and girls. Analysis of the intervention effect on screen time and sleep outcomes according to gender was specified in the protocol, ${ }^{18}$ however only one study ${ }^{87}$ reported separate outcomes for boys and girls, therefore subgroup analysis for gender was not possible. While Tamura and Tanaka ${ }^{87}$ reported sleep duration separately for girls and boys, no analysis of the results was completed nor discussed. 


\subsection{Intervention effect and screen time and sleep guidelines}

\section{Screen time}

Mean daily screen time hours at baseline across studies ranged from one hour to 2.8 hours per day, with the exception of one study reporting over four hours mean viewing per day. ${ }^{85}$ This indicates that, on average, randomised participants in all studies were exceeding the recommended screen-time hours for age at baseline. When considering the AAP screen time recommendations for age ${ }^{68,69}$ in the current review, participants were likely in excess of recommended limits at baseline. Post intervention, children aged two to six years continued to be in excess of the onehour daily limit, while children aged eight to 13 reduced viewing hours to the previous AAP recommended limit of two hours or less. ${ }^{69}$

\section{Sleep guidelines}

Children's sleep requirements vary according to age. Based on the reported mean sleep duration at baseline, it is likely that at least some of the participants in the included studies were achieving adequate sleep prior to the intervention. ${ }^{72}$ When considering intervention effect in terms of meeting recommended sleep duration hours for age, post-intervention the number of studies whose participants on average met sleep requirements for age increased from three $85,97,98$ to five studies. ${ }^{84,85,88,97,98}$ Consideration for baseline sleep duration in relation to sleep requirements for age is important. The potential improvement in sleep duration among participants meeting the recommended sleep requirements for age may be limited, which may bias intervention effects toward zero. Ensuring similarity between treatment groups at baseline in sleep duration is essential, particularly where participants may experience a large variation in sleep duration. This would minimise the impact of potentially achieving no intervention effect due to participants already achieving optimal sleep duration, but also reduce the impact of the greater gains in sleep duration expected to be achieved in participants with marked sleep deprivation.

\subsection{Intervention effect and consideration of uncontrolled variables}

Given the association between screen time and sleep problems, more substantial gains in intervention effect may have been expected. However, as presented in 
chapter 1 , screen time alone is not the only screen use variable that may affect sleep. The content viewed, other sources of unaccounted-for screen time, such as background television, parental screen use and a multitude of other factors affect screen use and sleep. The potential impact of these factors on intervention effect are considered further.

\subsubsection{Background screen exposure}

The screen time estimates discussed earlier (See section 1.1; chapter 3, results section) do not include screen use during school or childcare, and do not include the presence of background television. Inclusion of background screen exposure would further increase total screen time, with reports of parents having the television switched on in the same room in which a child plays at least $50 \%$ of the time. ${ }^{99}$ While background or 'ambient' television is often not included in total screen time, it is reported separately in some studies. Bickham et al. ${ }^{86}$ reported a reduction in viewing television during mealtimes and a reduction in the presence of background television, though not significant. While it may be difficult to measure the presence of background television or other background screen use, it is important in providing greater detail on potential total screen time, ${ }^{99}$ and may assist in explaining why some interventions may not achieve a significant change in sleep if large amounts of background screen exposure have not been taken into consideration.

\subsubsection{Content viewed}

Only one study specifically addressed the nature of the content viewed or attempted to control the content viewed. ${ }^{62}$ Media content is an important factor, particularly where there may be high background screen exposure ${ }^{99}$ and content is adultdirected (see section 1.1). Future research should ensure that the type of content viewed is recorded to allow appropriate analysis or that content is controlled by providing lists of appropriate children's programs, as well as education on program ratings. This is illustrated in the study by Garrison and Christakis, ${ }^{62}$ whereby in reducing violent media content and not limiting screen time, an improvement in children's sleep was observed. 


\subsubsection{Parenting self-efficacy and parental modelling}

Behaviour change is frequently the focus of intervention programs, such as reducing obesity or attempting to remove electronic media from the sleep environment; other factors associated with excessive screen time and sleep insufficiency in children are notably absent in research. Some risk factors are difficult to modify, such as socioeconomic status and parental educational attainment, however, there are other more-easily modifiable risk factors that should be taken into consideration. These include parental self-efficacy in controlling children's screen use, ${ }^{30}$ addressing household chaos, ${ }^{31}$ such as by introducing strategies for establishing family routine; and parental use of electronic media. ${ }^{30,31}$ While these factors were not listed as outcomes of interest in the current review, parental self-efficacy and parental screen use were evaluated in some of the included studies. Mostly these factors are rarely assessed. ${ }^{100}$ Evaluating parenting self-efficacy in relation to modifying their child's diet, activity and screen-based behaviour was addressed by Hammersley et al., ${ }^{100}$ however no significant change in parenting self-efficacy was observed in parents' perceived ability to manage each of these behaviours, except with their child's diet, potentially due to greater intervention content devoted to healthy eating than the other behaviours. ${ }^{100}$

Krossbakken et al..$^{90}$ also attempted to assess any change in parental self-efficacy after parents were provided with a guidebook on preventing problematic videogaming. Parents rated how certain they were in their ability to set and enforce limits to screen time and videogame time, however, no significant change was observed. ${ }^{90}$ Specifically incorporating strategies to improve parenting self-efficacy was an important component of the intervention evaluated by Walton et al. ${ }^{97}$ which was reflected in the results, finding a reduction in parental stress and improvement in parental warmth and parental self-efficacy post-intervention compared to within and between treatment groups.

The reduction in parental stress achieved is encouraging, given its association with higher screen time in children (see section 1.1.4). It supports the importance of incorporating parenting skills and stress reduction in interventions aiming for behaviour change, rather than simply aiming to educate parents in the potential harms of excessive screen time and thus change parental attitudes to screen time. Some parents may have a positive attitude toward reducing screen time but lack the 
ability to carry out screen-time reduction recommendations due to high parental stress levels or poor parental self-efficacy. Indeed, the inability of studies to elicit desired change in screen use could potentially be due to the intervention being unsuitable for the needs of parents experiencing high stress levels, and few of the included studies collected base line data to determine the presence of parental stress levels. Distribution of the number of parents experiencing high stress between control and intervention groups is unknown in the present review and may have affected the parents' attitudes toward the intervention, compliance and reporting of screen time hours. Screening parental stress levels at baseline and tailoring interventions for screen-time reduction in these households has been previously recommended. ${ }^{33}$

Parental modelling of desired behaviour in relation to screen use $\mathrm{s5,100,101}$ and sleep ${ }^{101}$ was measured in three included studies in this review. No significant change to parental screen time was reported by Hammersley et al., ${ }^{100}$ while Tomakyo et al ${ }^{101}$ found a greater reduction in total screen time in the intervention group compared to control, though not statistically significant, and no change between groups for weekday sleep duration, and some improvement in weekend sleep duration.

Parental sedentary behaviour was measured by Maddison et al. ${ }^{85}$ again showing no significant differences between intervention and control groups after the intervention. It is plausible that interventions aiming to reduce screen time may be more effective when designed for greater parental involvement. Future research specifically targeting parenting self-efficacy and limit setting, and aiming for a reduction in parental screen time is warranted, and should include measuring the child's screen time to determine if restricting parental screen time alone will, in turn, reduce children's screen time. Recognising the influence of the aforementioned factors in targeting and recruiting at-risk families, and addressing and evaluating these factors within an intervention, would potentially enhance program success in achieving desired behaviour change.

\subsection{Sustainability and reporting of harms}

The studies analysed offered no indication of the presence or absence of any harmful events resulting from the interventions. Measuring potential detrimental 
effects would offer insight into the barriers of changing screen-use behaviour, and inform future research. This may be particularly important in interventions targeting children exhibiting excessive screen use or signs of problematic videogaming or IGD. It has been suggested that studies investigating a reduction in or abstinence from electronic media in adolescents and children displaying excessive screen use and high levels of internet gaming should include psychiatric observation and standardised measurement of withdrawal symptoms during the intervention period as well as during a follow-up period. ${ }^{102}$ This highlights the need to ensure studies include follow-up measures beyond intervention completion, measurement of which was lacking among the studies presented in this review. Sustainability of results was only measured in three of the studies, showing a trend for the intervention effect to be sustained over a period of three to eight months following intervention completion.

\subsection{Limitations}

\subsubsection{Within-study limitations}

A significant limitation among all included studies is the potential risk of bias, particularly detection bias and social desirability bias, with the heavy reliance on participant reporting of outcomes using questionnaires of often-unknown reliability or validity. For both screen use and sleep outcomes, all included studies used questionnaires that were largely retrospective and completed by the participant or the participant's carer. Examples of types of questions used to measure sleep and screen use are provided in Appendix VI. While limitations are described briefly in the systematic review (chapter 3), further consideration of the limitations pertaining to defining and measuring screen time and sleep is warranted.

\section{a. Defining and measuring screen use}

Attempting to measure screen time was once relatively simple, as it was initially limited to the television and then the computer. With the development of mobile devices specifically designed for children, and with these screens being highly mobile and accessible, measuring screen use in children is now considerably harder. While estimating screen time is important, measuring screen time alone ignores other vital screen-use behaviours that have the potential to affect outcomes besides 
sedentary behaviour and BMI, such as sleep. Reporting screen use needs to incorporate other aspects of screen use besides total screen time, such as where, when and how the screen is used, and what content is being viewed in order to enable more thorough analysis of how these variables affect sleep.

Defining and reporting screen time hours between the included studies was inconsistent. Some studies provided separate screen-time hours for each device or activity, such as videogaming, internet, television, and specified time of day or weekday versus weekend. Some studies defined 'internet use' as time spent using a computer. No studies measured smartphone use. Other studies reported total screen time only, with or without providing detail of what screens were included. Some studies attempted to measure 'ambient' or 'background' television but neglected to consider other types of 'background' screen exposure, such as exposure to caregiver or sibling use of mobile devices or videogaming.

Few studies addressed the potential impact of the content viewed on the screen. Given the importance of the various types of content viewed and its potential to influence outcomes such as sleep and behaviour, there is a need for categorising the nature of screen use and content. Common terms include 'passive' or 'interactive' viewing of screens, but this is overly simple. Rideout ${ }^{1}$ proposes four categories: 'passive consumption', defined as viewing screen content only, such as watching TV; 'interactive consumption' such as playing videogames or 'surfing the internet'; 'communication', involving communicating with others via SMS, Skype or social media; and 'content creation', where new content is created by the user via a screen device, such as writing, art or music. These categories do not address the issue of background-screen exposure, nor the content viewed (violent versus ageappropriate) or the type of screen viewed, each of which have been shown to influence sleep. It may also be necessary to include overarching categories of recreational and non-recreational screen use. ${ }^{45}$

Greater objectivity in measuring screen use is required. Most studies utilised questionnaires of unknown reliability and validity, which rely on participant recall, often in retrospect. The use of smartphone applications for tracking screen time hold promise, such as the Apple Screen Time app, which offers a breakdown of screen time for categories of social networking, entertainment or productivity, or the use of 'battery use screen shot' for measuring smartphone usage. ${ }^{103}$ Until a 
method has been developed that objectively capturers the nature of screen use across multiple screen devices, it will be difficult to determine the effectiveness of interventions aiming to control screen use.

\section{b. Sleep duration as an outcome}

Of the lifestyle interventional studies that included sleep as an outcome, sleep duration was often the only sleep-related outcome reported. Although this may provide some insight into sleep, it oversimplifies the many factors that determine sleep sufficiency and quality and assumes that the child does not wake during the night. To avoid the potentially misleading use of 'sleep duration', some studies reported 'time in bed'. Focusing only on sleep duration or time in bed neglects to address important factors that may alter sleep, such as the number and duration of night wakings, the presence of any external sleeping aids, occurrence of night terrors, bedtime, bedtime resistance, sleep latency or co-sleeping. Sleep duration alone does not provide information on where a potential increase in sleep duration occurred, for example, an advancement in bedtime or later morning wake time.

In studies that evaluated interventions specifically targeting sleep, 'sleep duration' was one of many different sleep-related outcomes reported. Indeed, sleepspecific research seldom includes 'sleep duration' or does not rely on this measure as its main outcome. There is a need to supplement sleep duration with other sleep measures, such as the level of tiredness upon waking, or day-time sleepiness, which are indictors of whether the amount of sleep a child obtains is sufficient for that child.

Another issue with relying on 'sleep duration' as an outcome measure for sleep is the presence of optimal sleep sufficiency at baseline. If the participants are already achieving the recommended amount of sleep for age at baseline, then expecting to detect a change or achieve an increase in sleep duration hours is unlikely, thus highlighting the importance of utilising additional sleep outcomes. This may have affected the results of the review, with participants of three studies, on average, meeting sleep requirements, and the participants of another three studies on the cusp of meeting sleep requirements for age. While some studies stratified participants according to demographic variables, weight status, age and 
gender, some did not address baseline sleep duration, and instead controlled for any detected differences at baseline in subsequent analyses.

\section{c. Measurement of sleep duration}

Implementing a simple questionnaire may be the preferred choice of data collection due to its greater affordability and acceptability by the participant, as opposed to more invasive methods, however, greater objectivity is needed. For example, sleep outcomes are often derived from participant recall of waketime (the time at which the participant wakes up in the morning), bedtime, sleep onset latency, number of night-time wakings and, occasionally the duration of night wakings, recorded for weekend and/or weekday. Participants may estimate their own sleep and wake times, or studies may provide intervals from which participants can choose, (e.g. 6 a.m. to 6.30 a.m.). Such questionnaires are highly subjective and over-simplify the outcome of interest. Offering greater objectivity is actigraphy, providing the participant is comfortable with the requirements of the tool, or the use of smartphone applications that can measure sleep, however, the algorithms of the latter are not validated by scientific research. ${ }^{104}$ Indeed, a combination of participant report and objective measures would be ideal in providing greater insight into sleep and sleep-related outcomes; for example, self-reported sleep duration and quality have been found to correlate with self-reported health-related quality of life in children, while device-measured sleep duration did not. ${ }^{105}$ This supports the need for supplementing objectively measured sleep duration and sleep efficiency with other measures of sleep-related variables.

Some questionnaires utilised by the included studies were based on subsections of assessments with published tested reliability and validity, such as the Child Sleep Habits Questionnaire (CSHQ). However, it is uncertain if the validity and reliability of the measure is compromised when only using subsections and not completing the whole assessment. Others use questionnaires developed and presumably tested for validity and reliability by previous research and, finally, many of the outcomes were measured using questionnaires designed by those implementing the research, with no or minimal reporting of methods to determine inter-rater reliability or validity. 


\section{d. Timing of data collection}

Another important consideration in measuring screen use and sleep duration is the influence of time-use patterns over a week, season or year, with screen use and sleep patterns often being different on weekends compared to weekdays, dependent on special events being televised, or in school holidays compared to during the school term. Seasonality may also affect these behaviours, such as cold weather increasing time indoors, which may also result in an increase in screen time. This highlights the importance of utilising a control group and ensuring outcome measures for control and intervention groups are collected at the same time of week or school term and same time of year. Studies utilising a cross over design would be at particular risk of the issues mentioned above. The review presented in this thesis included studies utilising a control group in an attempt to mitigate the issues described here; however, included studies did not specifically state that the timing of data collection occurred at the exact same time between treatment groups. It is likely that the data was collected across a week or month, therefore not controlling for daily/weekly variation in screen-time habits.

\subsubsection{Limitations of the systematic review process}

Several limitations were identified pertaining to the processes utilised during the completion of this systematic review and are described briefly in the published review in chapter 3 , and discussed further here - in particular the review question and inclusion criteria, and the need to adjust data for pooled analysis. Regarding the review question, in limiting the population of interest to children aged two to 14 years, many relevant studies specifically targeting screen use were excluded (see Appendix V). Broad inclusion criteria for interventions targeting screen use resulted in the inclusion of lifestyle or health-promotion interventions targeting multiple behaviours for change, one of which was screen time. Many of these studies had been evaluated elsewhere in systematic reviews seeking to determine the effect of screen time reduction on BMI. With multiple other strategies included in the interventions that potentially impact sleep outcomes, it was impossible to differentiate the impact of screen use on sleep from other confounders. If the population of interest was extended to include children up to the age of 18 , and the 
interventions evaluated were limited to those specifically targeting screen use only, greater insight into the outcomes of controlling screen use may have been achieved.

The review included studies that evaluated an intervention and compared the intervention effect with a control group. Studies evaluating treatment effect without a comparison group were excluded (see examples listed in Appendix V). While this increases the quality of study design and strengthens reliability of results, few studies examining the effect of controlled screen use on sleep exist and expanding inclusion criteria to studies without a control group would have potentially offered preliminary insight into the types of interventions targeting screen use and sleep, and potential intervention effect.

A limitation of this review was the need to adjust or exclude data to increase consistency and allow pooling of data for meta-analysis. While some calculations could be performed to combine data where it was sub-grouped according to age or gender, this could not be done for combining screen-time hours for different screen types. Therefore, the meta-analysis of 'total screen time' hours conducted in this review did not include screen time that was reported separately (other than television viewing hours), such as videogaming and internet, unless an overall total screen time was provided. Where studies provided separate screen-time hours for weekends and weekdays, only the hours reported for weekday screen time were entered in the meta-analysis. Due to these limitations of studies and the available dataset encountered, and decisions and processes required to address them towards conduct of the review, completion of GRADE Summary of Findings, as intended $a$ priori $^{18}$ (see chapter 2, section: methods), did not appear to be warranted. As further research and future synthesis (see section 4.7) is conducted in this field, presentation of succinct summary of findings will no doubt evolve to be informative.

\subsection{Implications for future research}

Based on the studies included in this review, it is recommended that the following factors be considered in the design and implementation of studies evaluating children's screen-use interventions. In relation to study design:

- consider whether cluster RCT is appropriate, and as it is a popular method of randomisation within studies implemented in the school setting, studies must ensure appropriate number of clusters and must account for clustering 
in analysis. Simply 'randomising' one school to the control group and another school to the treatment group is not an appropriate method of randomisation

- provide baseline data for demographic variables that may affect results, such as parental education, income, employment, age, ethnicity, marital status, number of children, age and gender of the child, child weight

- studies seeking to determine the effect that limiting screen time or improving sleep may have on behaviour or academic performance, need to measure and control for how sleep sufficiency and screen time both have the potential to affect behaviour (see section 1.2, 1.3.1).

- ensure data collection for control and intervention groups occurs at a similar time of year, week and school term to reduce the impact these factors may have on participants' behaviour patterns and subsequent results

- measure sustainability of results beyond intervention completion. There was inadequate follow-up of intervention effect past intervention completion among the included studies. It has been recommended that future studies consider the amount of ongoing support needed by children and families to sustain behaviour change $\mathrm{e}^{62,88,98}$ and to adapt to the needs of their child as the child grows and their media habits change, ${ }^{62}$ with some studies suggesting continuing support for the duration of the child's schooling. ${ }^{88,98}$

In relation to screen use:

- utilise a combination of objective and subjective outcome measures for the same outcome. Use of objective measures will improve confidence in results and supplementation with subjective measures will provide insight into participant perceptions and incidence of under- or over-reporting, or allow for inclusion of a range of screen devices that otherwise may not be captured within the objective measure.

- attempt to measure exposure to background screen use and the type of content to which the child is potentially exposed

- consider quantifying or defining screen-use variables by applying screenuse categories as proposed by Rideout ${ }^{1}$ and Babic et al. ${ }^{43}$

- screen for likelihood of prior exposure to violent screen content 
- include psychiatric observation and standardised assessment of withdrawal symptoms for participants receiving interventions that restrict screen access or completely eliminate screen use, particularly for participants exhibiting problematic or high screen use at baseline.

In relation to sleep:

- supplement measurement of 'sleep duration' with other sleep-related outcomes and document all details, including bedtime and waketime, night wakings, sleep onset latency and sleep deficit

- ensure study groups are similar at baseline for sleep parameters

- potentially target participants identified as experiencing sleep insufficiency, and if aiming to implement a health-promotion lifestyle intervention in healthy participants, use of other multiple sleep-related outcomes is essential. This may assist in avoiding under-detection of changes to participant sleep, as may happen if sleep duration is the only outcome measured.

\subsection{Implications for practice}

Based on the findings of the systematic review and meta-analysis presented in this thesis, interventions that aim to address screen-use behaviour, either as part of an intervention targeting screen use alone or within a multi-component intervention, can contribute to a reduction in screen time and improvement in sleep. The intervention effect appeared to be further strengthened in studies that employed certain intervention design and implementation strategies, with implications for the development of interventions targeting screen use in children. A summary of key intervention characteristics that appeared to strengthen the intervention effect include:

- a strong or primary focus on screen use and/or sleep

- a focus on either the prevention of excessive screen use, or intervention to reduce or modify screen-use behaviours in children exhibiting excessive or inappropriate screen use

- direct face-to-face contact with the child and their care giver

- tailoring the intervention to the participant's needs 
- utilising a range of behavioural modification strategies, such as goal setting, media budgeting, 'screen-free' challenges, alternative activities, screen time being contingent on participation in other activities, and peer monitoring of screen use to support the implementation of screen use and sleep recommendations and related educational material.

In developing interventions to address screen use in children, consideration should be given to factors that are associated with increased screen time or inappropriate screen use in children. While these factors were evaluated in narrative form only and based on the results of a limited number of studies, it may be beneficial to address the following in the development of interventions targeting screen use in children:

- remove bedroom televisions

- ensure sleep environments are screen-free

- incorporate parental screen use and modelling of desired screen use

- provide a break-down of baseline screen time, content and screen-type preferences

- screen for the presence of parental stress (financial, available time) and level of parental self-efficacy in managing their child's behaviour and provide additional support and intervention content tailored to the needs of at-risk families.

\subsection{Conclusion}

Interventions aiming to control screen use and improve sleep in children have the potential to achieve small reductions in screen time and gains in sleep duration, and can assist children in meeting recommended screen time and sleep guidelines for age. Due to the nature of screen use, lack of objective screen-use measures and inadequate controlling of multiple potentially confounding factors, the high heterogeneity shown in the meta-analysis was anticipated. When such factors are addressed and the interventions evaluated contain content targeting screen use alone, greater confidence in intervention effect and lower heterogeneity would be expected. While bedtime screen use appears to be associated with sleep insufficiency, causality cannot yet be claimed. There is a need for more RCTs that aim to specifically evaluate the effect controlling screen use has on sleep, utilising 
objective outcomes, before causality between inappropriate screen use (excessive, age-inappropriate content, or poorly timed screen use) and sleep problems in children can be determined. 


\section{Appendices}

\section{Appendix I: Search strategy}

\section{PsycINFO}

First search conducted 10/10/2017 yielded 292 results.

1. (child* or toddler* or pre-school* or preschool* or elementary school* or kindergarten or kindy or primary school* or middle school or adolescen* or boy or boys or girl or girls or parent* or family or families).ti,ab. or parents.sh. or exp childhood development/ 2. (screen time or screen-use or (mobile adj2 devices) or television or TV or touch screen* or touchscreen* or smart phone* or smartphone* or cell phone* or cellular phone* or cellphone* or mobile phone* or (hand held adj2 device*) or (handheld adj2 device*) or (electronic adj2 media) or computer or computer gaming or (computer adj3 game*) or social media or (social adj3 network*) or (internet adj3 addict*) or video game* or video gaming or online game* or online gaming or (media adj3 violence)).ti,ab. or social media.sh. or media exposure.sh. or screen time.sh. or sedentary behavior.sh. or mass media.sh. or media exposure.sh. or television.sh. or exp mobile devices/ or exp cellular phones/ or exp computer games/ or exp online social networks/ 3 . (counselling or intervention* or counseling or education or lifestyle or reduction or reduce or restrict* or limit* or media literacy or (parent* adj3 monitor*) or (parent* adj3 mediation) or coviewing or co-viewing or violence prevention or curriculum or health promotion or behavior change or control*).ti,ab. or exp behavior change/ or exp parenting/

4. (sleep* or insomnia or night waking or tiredness or bedtime or (bed adj3 time)).ti,ab. or sleep.sh.

5. 1 and 2 and 3 and 4

\section{PubMed}

First search conducted 10/10/2017 yielded 596 results. ((((sleep*[tiab] OR insomnia[tiab] OR “night waking"[tiab] OR tiredness[tiab] OR bedtime[tiab] OR sleep[mh] OR sleep hygiene[mh]))

AND

(reduction[tiab] OR reduce[tiab] OR restrict*[tiab] OR limit*[tiab] OR control*[tiab] OR counseling[tiab] OR counselling[tiab] OR education[tiab] OR lifestyle[tiab] OR "health promotion" [tiab] OR "media literacy"[tiab] OR intervention*[tiab] OR "behavior change"[tiab] OR "parental monitoring"[tiab] OR "parental mediation" [tiab] OR coviewing[tiab] OR “co viewing"[tiab] OR parenting[tiab] OR health promotion[mh])) AND

("screen time"[tiab] OR screen-use[tiab] OR "mobile device*"[tiab] OR television[tiab] OR TV[tiab] OR "touch screen*"[tiab] OR touchscreen*[tiab] OR "smart phone*”[tiab] OR smartphone*[tiab] OR "mobile phone*" [tiab] OR "hand held device*”[tiab] OR "handheld device*"[tiab] OR cellphone[tiab] OR "cell phone*"[tiab] OR "cellular phone*"[tiab] OR "electronic media" [tiab] OR computer[tiab] OR "computer game*"[tiab] OR “computer gaming"[tiab] OR "online game*” OR "online gaming" OR "online social network*" [tiab] OR "internet addiction" [tiab] OR "social media"[tiab] OR "video game*" [tiab] OR "video gaming" [tiab] OR "social network*"[tiab] OR "media violence"[tiab] OR "media exposure"[tiab] OR social media[mh] OR internet[mh] OR video games[mh] OR cell phones[mh]))

AND 
(child*[tiab] OR toddler*[tiab] OR "pre school"[tiab] OR preschool[tiab] OR kindergarten[tiab] OR kindy[tiab] OR "elementary school*"[tiab] OR "primary school" [tiab] OR "middle school"[tiab] OR adolescen*[tiab] OR family[tiab] OR families[tiab] OR parent*[tiab] OR girl[tiab] OR girls[tiab] OR boy[tiab] OR boys[tiab] OR parents[mh] OR child development[mh])

\section{Scopus}

First search conducted 10/10/2017 yielded 2045 results.

( TITLE-ABS-KEY ( child* OR toddler* OR preschool* OR "pre

school*" OR "elementary school*" OR kindergarten OR kindy OR "primary

school*" OR "middle

school*" OR adolescen* OR family OR families OR parent* OR boy OR boys $\mathrm{O}$ $\mathrm{R}$ girl OR girls ) )

AND

( TITLE-ABS-KEY ( sleep* OR insomnia OR "night waking" OR tiredness OR bedtime ) ) AND ( ( TITLE-ABS-KEY ( "violence prevention" OR curriculum )) OR ( TITLE-ABS-

KEY ( reduction OR reduce OR restrict* OR limit* OR control* OR counselling O $\mathrm{R}$ counselling OR lifestyle OR "health promotion" OR "media

literacy" OR intervention OR "behavior change" OR "parental monitoring" OR "parental mediation" OR coviewing OR "co viewing")) )

AND

( ( TITLE-ABS-KEY ( "screen time" OR "screen-use" OR "mobile

devices" OR television OR tv OR "touch screen*" OR touchscreen* OR "smart

phone*" OR smartphone* OR "mobile phone*" OR cellphone* OR "cell

phone*" OR "cellular phone*" OR "hand held device" OR "handheld

device" ) ) OR ( TITLE-ABS-KEY ( "electronic media" OR computer OR "computer game*" OR "computer gaming" OR "internet addiction" OR "social

media" OR "video game*" OR "video gaming" OR "online gaming" OR "online

game*" OR "social network*" OR "media violence" OR "media exposure")))

\section{ERIC}

First search conducted 10/10/2017 yielded 32 results.

(AB,TI(child*) OR AB,TI(toddler*) OR AB,TI(“pre school”) OR AB,TI(preschool) OR AB,TI("elementary school") OR AB,TI(kindergarten) OR AB,TI(kindy) OR

AB,TI(“primary school”) OR AB,TI(“middle school”) OR AB,TI(adolescen*) OR $\mathrm{AB}, \mathrm{TI}($ families) OR AB,TI(family) OR AB,TI(parent*) OR AB,TI(boy) OR AB,TI(boys) OR AB,TI(girl) OR AB,TI(girls))

AND

(AB,TI,FT(“screen time”) OR AB,TI,FT(“screen-use") OR AB,TI,FT(“mobile device*”) OR AB,TI,FT(television) OR AB,TI,FT(TV) OR AB,TI,FT("touch screen*”) OR AB,TI,FT(touchscreen*) OR AB,TI,FT(“smart phone*”) OR AB,TI,FT(smartphone*) OR AB,TI,FT("mobile phone*") OR AB,TI,FT("handheld device*”) AB,TI,FT("hand held device*”) OR AB,TI,FT(cellphone*) OR AB,TI,FT(“cell phone*”) OR AB,TI,FT("cellular phone*”) OR AB,TI,FT(computer) OR AB,TI,FT("electronic media") OR AB,TI,FT(“online social network*") OR AB,TI,FT("computer game*”) OR AB,TI,FT(“computer gaming") OR AB,TI,FT("internet addiction") OR AB,TI,FT("social media") OR AB,TI,FT("video gaming") OR AB,TI,FT("video game*”) OR AB,TI,FT(“online gaming”) OR AB,TI,FT(“online game*”) OR AB,TI,FT(“media violence") OR AB,TI,FT(“media exposure")) 


\section{AND}

(AB,TI(reduction) OR AB,TI(reduce) OR AB,TI(restriction) OR AB,TI(limit*) OR $\mathrm{AB}, \mathrm{TI}($ control*) OR AB,TI(counselling) OR AB,TI(counseling) OR AB,TI(education) OR AB,TI(lifestyle) OR AB,TI("health promotion") OR AB,TI(intervention) OR $\mathrm{AB}, \mathrm{TI}$ ("behavior change") OR AB,TI("parental monitoring") OR AB,TI("parental mediation") OR AB,TI(coviewing) OR AB,TI(“co viewing”) OR AB,TI,FT(“media literacy") OR AB,TI,FT(“violence prevention”) OR AB,TI(curriculum))

AND

(AB,TI,FT(sleep*) OR AB,TI(insomnia) OR AB,TI,FT(tiredness) OR AB,TI,FT("night waking") OR AB,TI,FT(bedtime))

\section{Embase}

\section{\#2 AND \#3 AND \#4 AND \#5}

First search conducted 10/10/2017 yielded 635 results \#5 sleep:ti,ab OR insomnia:ti,ab OR bedtime:ti,ab OR tiredness:ti,ab OR 'night waking':ti,ab

218,777

\#4 reduction:ti,ab OR reduce:ti,ab OR restriction:ti,ab OR limit*:ti,ab OR control*:ti,ab OR counselling:ti,ab OR counseling:ti,ab OReducation:ti,ab OR lifestyle:ti,ab OR 'health promotion':ti,ab OR 'media exposure':ti,ab OR 'media literacy':ti,ab OR intervention:ti,ab OR'behavior change':de,ti,ab OR 'parental monitoring':ti,ab OR 'parental mediation':ti,ab OR 'co viewing':ti,ab OR coviewing:ti,ab ORcurriculum:ti,ab OR 'violence prevention':ti,ab

$7,540,803$

\#3 'screen time':ti,ab OR 'screen-use':ti,ab OR 'mobile device*':ti,ab

OR television:de,ti,ab OR tv:ti,ab OR 'touch screen*':ti,ab ORtouchscreen*:ti,ab OR 'smart phone*':ti,ab OR smartphone*:ti,ab OR 'mobile phone*':de,ti,ab OR 'hand held device*':ti,ab OR 'handheld device*':ti,ab OR 'cellular phone*':ti,ab OR cellphone*:ti,ab OR 'cell phone*':ti,ab OR computer:ti,ab OR 'electronic media':ti,ab OR 'online social networks':ti,ab OR 'computer game*':ti,ab OR 'computer gaming':ti,ab OR 'internet addiction':ti,ab OR 'social media':de,ti,ab OR'computer addiction':de,ti,ab OR 'video game*':ti,ab OR 'video gaming':ti,ab OR 'media violence':ti,ab

298,845

\#2 ((child*:ti,ab OR toddler*:ti,ab OR 'childhood'/exp OR childhood)

AND development:de OR pre) AND school:ti,ab OR family:ti,ab ORfamilies:ti,ab OR adolesc*:ti,ab OR preschool*:ti,ab OR 'elementary school*':ti,ab OR 'middle school*':ti,ab OR 'primary school*':ti,ab ORkindergarten:ti,ab OR kindy:ti,ab OR parent*:ti,ab OR boy:ti,ab OR boys:ti,ab OR girls:ti,ab OR girl:ti,ab $1,794,522$

\#1 ((child*:ti,ab OR toddler*:ti,ab OR 'childhood'/exp OR childhood) AND development:de OR pre) AND school:ti,ab OR family:ti,ab ORfamilies:ti,ab OR adolesc*:ti,ab OR preschool*:ti,ab OR 'elementary school*':ti,ab OR 'middle school*':ti,ab OR 'primary school*':ti,ab ORkindergarten:ti,ab OR kindy:ti,ab OR parent*:ti,ab OR boy:ti,ab OR boys:ti,ab OR girls:ti,ab OR girl:ti,ab 


\section{Appendix II: Critical appraisal tool}

\section{a. JBI critical appraisal tool for randomised controlled trials}

Date:

Study:

1. Was true randomisation used for assignment of participants to treatment groups?

Yes $\quad$ Method by which randomisation to intervention or control group described (e.g., random allocation using number generator, stratified block randomisation scheme).

No Method other than randomisation used to allocate patients to groups (e.g., quasi randomisation/stratification).

Unclear Terms like 'random' and 'randomisation' used but method not described.

Comment/detail

\section{Was allocation to treatment groups concealed?}

Yes The researcher responsible for allocating participants to compared groups is unaware of the allocation order. An appropriate allocation concealment method was used, such as central randomisation; sequentially numbered opaque sealed envelopes used?

No Person responsible for allocation to groups able to determine which group the participant was being allocated.

Unclear Unable to determine how allocation to treatment groups occurred.

Comment:

\section{Were treatment groups similar at the baseline?}

Yes

Recorded minimum base line data including:

- Age

- Sex

- SES indicators: parental education level, or household income

- Electronic screen use/exposure

- Baseline measurements for individual intended measurable outcomes.

Additional information can include:

- Geographical location

- Employment status of parent/carer

- Ethnicity

- Family members (e.g., siblings, single parent).

No Baseline data between groups is clearly not comparable (statistical differences between groups at baseline that may affect the outcome of results, e.g., differences in sex, age, SES on effectiveness of intervention/uptake).

Unclear Inadequately described. No or minimal reporting of baseline data (i.e. only age, sex). No mention of statistical difference between groups where differences in baseline data are apparent.

Comment:

4. Were participants blind to treatment assignment? 
Yes Participants unaware that they have been allocated to either intervention or control group and methods for ensuring participant blinding to treatment assignment indicated.

No Participants aware of which group they have been allocated.

Unclear Inadequately described.

Comment:

5. Were those delivering the treatment blind to treatment assignment?

Yes Therapists/teachers/educators implementing the intervention are unaware if they are providing intervention to control or intervention/treatment group.

No Therapists/teachers/educators implementing the intervention are aware they are providing the intervention to the treatment group Provide explanation for lack of blinding.

Unclear Inadequately described.

Comment:

6. Were outcomes assessors blind to treatment assignment?

Yes Data collectors were blinded for outcomes assessment (e.g., conducting interview).

No Data collectors were aware of the group in which the participant belonged.

Comment:

Unclear Inadequately described.

7. Were treatment groups treated identically other than the intervention of interest?

Yes Participants in both the intervention and control groups were treated identically for all other aspects other than intervention of interest (e.g., control group received standard curriculum if the intervention occurred in the school setting).

Study includes active control group. Any intervention provided to control group described in detail (e.g., receiving usual health promotion printed handouts, matched for attention/program intensity).

No Wait-listed control groups where control intervention not provided, or intervention provided clearly not matched for attention, giving control participants an indication that they may be in the control group.

Participants receiving concurrent intervention outside intervention of interest that may impact on results (e.g., attending extracurricular activities that may reduce screen time, such as school camp).

Unclear Control intervention inadequately described.

Comment:

8. Was follow-up complete and if not, were differences between groups in terms of their follow-up adequately described and analysed?

Yes Complete follow up.

Withdrawn participants/losses to follow-up reported and reasons for the withdrawal described.

All participants included in final calculations including withdrawn participants, regardless of whether their final outcomes were measured. 
No No explanation of withdrawn participants/losses to follow-up or the significance of these withdrawals

Withdrawn participants not analysed in the groups to which they were allocated

Unclear Withdrawn participants inadequately described

Numbers of included/withdrawn participants do not match result

Comment:

figures; inadequately described

9. Were participants analysed in the groups to which they were randomised?

Yes Withdrawn participants analysed in the groups to which they were

originally allocated (intention to treat analysis, ITT)

No Missing participant data not reported or accounted for

Unclear Inadequately described

Comment:

10. Were outcomes measured in the same way for treatment groups?

Yes Outcome data was measured and collected consistently in all groups

No Outcome data was measured and collected differently for each group

Unclear Inadequately described

Comment:

11. Were outcomes measured in a reliable way?

Yes Outcomes (sleep duration/quality/bedtime, screen time/screen content viewed/time of viewing) measured using standardised methods

- Authors mention the reliability and/or validity of the measures used (incl trained data collectors) or piloted within the trial.

- Demonstrates/indicates test-retest reliability

- E.g., Actigraphy

No Self-reported/subjective outcomes, reliant on participant recall

- $\quad$ Sleep or ST diary

- Parent report of bedtime/wake time

- $\quad$ Child Sleep Habits Questionnaire

No reporting on the reliability and/or validity of the methods used for measuring outcome or if training was provided for data collectors

Unclear Inadequately described

Comment:

12. Was appropriate statistical analysis used?

Yes Appropriate statistical methods used, described and reported

No Statistical methods inappropriate

Unclear Inadequately described

Comment:

13. Was the trial design appropriate and any deviations from the standard RCT design (individual randomisation, parallel groups) accounted for in the conduct and analysis of the trial? 
Yes Described study methods in detail and any deviation from standard RCT design accounted for with explanation

No Inappropriate study design/no explanation of deviation from standard RCT

Unclear Inadequately described, lack of detail

Comment: 


\section{Appendix II: Critical appraisal tool}

\section{b. JBI critical appraisal tool quasi-experimental studies}

\section{Date: Study:}

1. Is it clear in the study what is the 'cause' and what is the 'effect' (i.e. there is no confusion about which variable comes first)?

Yes

No

Unclear

2. Were the participants included in any comparisons similar?

Yes

No

Unclear

3. Were the participants included in any comparisons receiving similar treatment/care, other than the exposure or intervention of interest?

Yes

No

Unclear

4. Was there a control group?

Yes

No

Unclear

5. Were there multiple measurements of the outcome both pre- and post- the intervention?

Yes

No

Unclear

6. Was follow-up complete and if not, were differences between groups in terms of their follow-up adequately described and analysed?

Yes

No

Unclear

7. Were the outcomes of participants included in any comparisons measured in the same way?

Yes

No

Unclear

8. Were outcomes measured in a reliable way?

Yes

No

Unclear

9. Was appropriate statistical analysis used?

Yes

No

Unclear 


\section{Appendix III: Data extraction forms}

Year:

Study title:

Author:

Country:

Study design:

Participant age range:

Mean age

SD

Gender

Demographics: ethnic minority/ baseline screen use/ overweight

Treatments groups similar at baseline for outcomes and demographic variables?

Setting: home/school/community

\begin{tabular}{l|l|l|l} 
Sample size at & Control & Intervention & Total \\
\hline baseline & & & \\
\hline point of randomization & & & \\
\hline intervention completion & & &
\end{tabular}

Study aim:

Powered to detect change in:

Intervention description:

Intervention duration:

Frequency of contact:

Control group details:

Number of sessions on screen use:

Number of sessions on sleep:

Provided: to carer/ child

Delivery method: face to face/ Group/ mail online/ both

Group/ individual

Outcomes overall:

Screen use-related outcomes:

Outcome /unit of measure

Outcome measure assessment/tool

Sleep-related outcomes:

Outcome /unit of measure

Outcome measure assessment/tool 
Study results:

\begin{tabular}{|c|c|c|c|c|c|c|c|}
\hline \multirow[b]{2}{*}{ Outcome } & \multicolumn{3}{|c|}{ Intervention $\mathrm{n}$ : } & \multicolumn{3}{|c|}{ Control n: } & \multirow[b]{2}{*}{$\begin{array}{l}\text { MD, } \\
(95 \% \\
\text { CI) }\end{array}$} \\
\hline & $\begin{array}{l}\text { Mean } \\
\text { Pre } \\
(\mathrm{SD})\end{array}$ & $\begin{array}{l}\text { Mean } \\
\text { Post } \\
\text { (SD) }\end{array}$ & $\begin{array}{l}\text { Mean } \\
\text { change } \\
\text { (SD) }\end{array}$ & $\begin{array}{l}\text { Mean } \\
\text { Pre } \\
(\mathrm{SD})\end{array}$ & $\begin{array}{l}\text { Mean } \\
\text { Post } \\
\text { (SD) }\end{array}$ & $\begin{array}{l}\text { Mean } \\
\text { change } \\
\text { (SD) }\end{array}$ & \\
\hline & & & & & & & \\
\hline & & & & & & & \\
\hline & & & & & & & \\
\hline & & & & & & & \\
\hline
\end{tabular}

Additional findings: 


\section{Appendix IV: Statistical methods}

\section{a. Estimating the correlation coefficient for pre- and post-intervention measures of sleep duration}

Prepared for: Katie Martin

Date: 08/09/2018

Statistician: Jana Bednarz

Using Haines ${ }^{84}$ paper as example (and looking at the sleep duration outcome

measurement):

For each of the intervention and control groups, the following information is known:

- Mean (SD) sleep duration at baseline

- Mean (SD) sleep duration at six months

- Change (SD) in sleep duration (six months minus baseline)

\section{Intervention group:}

$X$ mean outcome at baseline $($ pre $)=10.52$

$Y$ mean outcome at follow-up (post) $=11.07$

mean change in the outcome denoted $(Y-X)=0.56$

The equation to estimate the correlation between $X$ and $Y$ :

Therefore:

$$
\operatorname{Corr}(X, Y)=\rho_{X Y}=\frac{\operatorname{Cov}(X, Y)}{\sigma_{X} \sigma_{Y}}
$$

The value for $\sigma_{X}$, the standard deviation for $X=1.42$ (from the table)

The value for $\sigma_{Y}$, the standard deviation for $Y=1.45$

Need a value for $\operatorname{Cov}(X, Y)$.

The equation for the variance of the change:

$$
\operatorname{Var}(Y-X)=\operatorname{Var}(Y)+\operatorname{Var}(X)-2 \operatorname{Cov}(X, Y)
$$

Rearrange this equation to make $\operatorname{Cov}(X, Y)$ the subject:

Substituting these values into the equation:

$$
\begin{gathered}
\operatorname{Cov}(X, Y)=\frac{1}{2} \times[\operatorname{Var}(X)+\operatorname{Var}(Y)-\operatorname{Var}(Y-X)] \\
\operatorname{Var}(X)=\sigma_{X}^{2}=(1.42)^{2} \\
\operatorname{Var}(Y)=\sigma_{Y}^{2}=(1.45)^{2} \\
\operatorname{Var}(Y-X)=(1.6)^{2}
\end{gathered}
$$

$$
\begin{gathered}
\operatorname{Cov}(X, Y)=\frac{1}{2}\left[(1.42)^{2}+(1.45)^{2}-(1.6)^{2}\right] \\
\operatorname{Cov}(X, Y)=0.77945
\end{gathered}
$$

This provides the value for the $\operatorname{Corr}(X, Y)$ equation:

Substituting:

$$
\operatorname{Corr}(X, Y)=\frac{\operatorname{Cov}(X, Y)}{\sigma_{X} \sigma_{Y}}
$$

$$
\begin{gathered}
\operatorname{Cov}(X, Y)=0.77945 \\
\sigma_{X}=1.42 \\
\sigma_{Y}=1.45
\end{gathered}
$$

Therefore: 


$$
\begin{gathered}
\operatorname{Corr}(X, Y)=\frac{0.77945}{1.42 \times 1.45} \\
\operatorname{Corr}(X, Y)=0.3786
\end{gathered}
$$

\section{Control group:}

$X($ pre $)=10.38$

$Y($ post $)=10.16$

Mean change $(Y-X)=-0.19$

Standard deviation for $\mathrm{X}=\sigma_{X}=1.63$ (from the table)

Standard deviation for $\mathrm{Y}=\sigma_{Y}=1.6$

To find $\operatorname{Cov}(X, Y)$ :

$$
\operatorname{Corr}(X, Y)=\rho_{X Y}=\frac{\operatorname{Cov}(X, Y)}{\sigma_{X} \sigma_{Y}}
$$

$$
\operatorname{Cov}(X, Y)=\frac{1}{2} \times \operatorname{Var}(X)+\operatorname{Var}(Y)-\operatorname{Var}(Y-X)
$$

Where:

$$
\begin{gathered}
\operatorname{Var}(X)=\sigma_{X}^{2}=(1.63)^{2} \\
\operatorname{Var}(Y)=\sigma_{Y}^{2}=(1.6)^{2} \\
\operatorname{Var}(Y-X)=(2)^{2}
\end{gathered}
$$

Therefore:

$$
\begin{gathered}
\operatorname{Cov}(X, Y)=\frac{1}{2} \times\left[(1.63)^{2}+(1.6)^{2}-(2)^{2}\right] \\
\operatorname{Cov}(X, Y)=0.60845
\end{gathered}
$$

Now returning to the equation for the correlation:

Substituting:

$$
\operatorname{Corr}(X, Y)=\frac{\operatorname{Cov}(X, Y)}{\sigma_{X} \sigma_{Y}}
$$

Therefore:

$$
\begin{gathered}
\operatorname{Cov}(X, Y)=0.60845 \\
\sigma_{X}=1.63 \\
\sigma_{Y}=1.6
\end{gathered}
$$

$$
\begin{gathered}
\operatorname{Corr}(X, Y)=\frac{0.60845}{1.63 \times 1.6} \\
\operatorname{Corr}(X, Y)=0.2333
\end{gathered}
$$




\section{b. Calculating the variance for mean change}

Working example prepared by Jana Bednarz:

For Maddison et al. ${ }^{85}$

Intervention group:

$\begin{array}{lll}X=640 & \text { sd } 85 & \operatorname{Var}(X)=0.86^{2} \\ Y=645 & \text { sd } 95 & \operatorname{Var}(Y)=0.80^{2}\end{array}$

Mean change $(Y-X)=5$

$\operatorname{Corr}(X, Y)=0.3786$

$$
\operatorname{Var}(Y-X)=\operatorname{Var}(Y)+\operatorname{Var}(X)-2 \operatorname{Cov}(X, Y)
$$

$\sigma_{X}=85$ (standard deviation of $\left.X\right)$

$\operatorname{Var}(X)=85^{2}$ (variance of $\left.X\right)$

$\sigma_{Y}=95(S D$ of $Y)$

$\operatorname{Var}(Y)=95^{2}($ variance of $Y)$

$\operatorname{Corr}(X, Y)=0.3786$

To solve for $\operatorname{Cov}(X, Y)$ :

$$
\begin{gathered}
\operatorname{Cov}(X, Y)=\operatorname{Corr}(X, Y) \times \sigma_{X} \times \sigma_{Y} \\
\operatorname{Cov}(X, Y)=(0.3786 \times 85 \times 95)
\end{gathered}
$$

Putting these elements into the equation for $\operatorname{Var}(Y-X)$ :

$$
\begin{gathered}
\operatorname{Var}(Y-X)=95^{2}+85^{2}-[2 \times(0.3786 \times 85 \times 95)] \\
\operatorname{Var}(Y-X)=10135.61 \\
S D=\sqrt{10135.61} \approx 100.7 \text { minutes }
\end{gathered}
$$

Converting into hours

$(Y-X)=\frac{5}{60}=0.083$ hours

$S D=\frac{\sqrt{10135.61}}{60}=1.68$ hours 


\title{
c. Calculating the Standard Deviation from the 95\% Confidence Interval for the Mean for a Continuous Outcome for One Sample
}

\author{
Prepared by Jana Bednarz
}

The formula for the $95 \%$ confidence interval for the population mean for continuous outcome $\mathrm{X}$ is:

where

$$
\bar{X} \pm\left(1.96 \times \frac{s}{\sqrt{n}}\right)
$$

$\bar{X}$ is the sample mean

$s$ is the sample standard deviation

$n$ is the sample size

Rearrange this equation to make $s$ the subject. First, define the equation for the lower bounds of the $95 \% \mathrm{CI}$ :

Lower 95\% Confidence Limit (LCI):

Making s the subject in the equation:

$$
\bar{X}-\left(1.96 \times \frac{s}{\sqrt{n}}\right)
$$

$$
\begin{gathered}
L C I=\bar{X}-\frac{1.96 s}{\sqrt{n}} \\
\bar{X}-L C I=\frac{1.96 s}{\sqrt{n}} \\
\sqrt{n}(\bar{X}-L C I)=1.96 s \\
\rightarrow s=\frac{\sqrt{n}(\bar{X}-L C I)}{1.96}
\end{gathered}
$$

To find s, substitute into the equation above the reported values for:

$\bar{X}=$ the reported mean

$L C I=$ the lower bound of the $95 \% \mathrm{CI}$ for the mean

$n=$ the sample size 


\section{Calculating standard deviation from $95 \%$ CI for Bickham, ${ }^{78}$ working} example:

Intervention group pre-test

$\bar{X}$ is the sample mean $=8.5 \times 60=510$

$S$ is the sample standard deviation $=$ ?

$n$ is the sample size $=143$

$L C I=8.3 \times 60=498$

$$
\begin{gathered}
\rightarrow s=\frac{\sqrt{n}(\bar{X}-L C I)}{1.96} \\
\rightarrow s=\frac{\sqrt{143(\overline{510}-498)}}{1.96} \\
=73.212
\end{gathered}
$$

Convert to hours: $73.212 / 60=1.22$ hours

Post-test:

$\bar{X}$ is the sample mean $=8.7 \times 60=522$

$s$ is the sample standard deviation $=$ ?

$n$ is the sample size $=143$

$L C I=8.4 \times 60=504$

$$
\begin{aligned}
\rightarrow s & =\frac{\sqrt{143}(\overline{522}-504)}{1.96} \\
& \rightarrow s=\frac{215.244}{1.96} \\
& \rightarrow s=109.82
\end{aligned}
$$

Convert to hours: $\rightarrow s=1.83$ hours

Control group pre-test:

$\bar{X}$ is the sample mean $=8.4 \times 60=504$

$S$ is the sample standard deviation $=$ ?

$n$ is the sample size $=269$

Lower CI

$$
=8.3 \times 60=498
$$

$$
\begin{aligned}
\rightarrow s= & \frac{\sqrt{269}(\overline{504}-498)}{1.96} \\
& \rightarrow s=\frac{16.4(6)}{1.96} \\
& \rightarrow s=50.20
\end{aligned}
$$

Convert to hours: $\rightarrow s=0.83 \mathrm{hrs}$

Control group post-test:

$\bar{X}$ is the sample mean $=8.2 \times 60=492$

$s$ is the sample standard deviation $=$ ?

$n$ is the sample size $=269$

Lower CI $=8.1 \times 60=486$

$$
\begin{gathered}
\rightarrow s=\frac{\sqrt{269}(\overline{492}-486)}{1.96} \\
\rightarrow s=50.20
\end{gathered}
$$




\section{Appendix V: Excluded studies}

The following studies were excluded following full text retrieval:

1. Michels N, De Henauw S, Eiben G, Hadijgeorgiou C, Hence S, Hensberger M, et al. Effect of the IDEFICS multilevel obesity prevention on children's sleep duration. Obesity Reviews 2015;16(suppl 2):68-77.

Screen time not measured (presence of bedroom television only)

2. Händel MN, Larsen SC, Rohde JF, Stougaard M, Olsen NJ, et al. Effects of the Healthy Start randomized intervention trial on physical activity among normal weight preschool children predisposed to overweight and obesity. PLoS ONE 2017;12(10): e0185266.

No outcomes of interest

3. Olsen NJ, Buch-Andersen T, Händel MN, Østergaard LM, Pedersen J, Seeger C, et al. The Healthy Start project: a randomized, controlled intervention to prevent overweight among normal weight, preschool children at high risk of future overweight. BMC Public Health 2012;12(1):590.

Duplicate: same data as Handel 2017; does not measure screen time

4. Kolaitis G, Korpa T, Papadopoulou P. A health promotion program for children and adolescents with ADHD. European Child \& Adolescent Psychiatry 2015

Outcomes of interest not measured

5. Barbosa Filho V, Lopes A, Lima A, de Souza EA, Gubert F, Silva KS, et al. Rationale and methods of a cluster-randomized controlled trial to promote active and healthy lifestyles among Brazilian students: the "Fortaleça sua Saúde" program. BMC Public Health 2015;15:1212.

Outside age range (11 to 18 years); outcome of interest not measured

6. Bartel K, Scheeren R, Gradisar M. Altering adolescents' pre-bedtime phone use to achieve better sleep health. Health Communication 2019;34(4):456-462.

Outside age range (14 to 18 years)

7. Raat H, Struijk MK, Remmers T, Vlasblom E, van Greiken A, Broeren S, et al. Primary prevention of overweight in preschool children, the BeeBOFT study (breastfeeding, breakfast daily, outside playing, few sweet drinks, less TV viewing): design of a cluster randomized controlled trial. BMC Public Health 2013;13:974.

Outside age range (birth to three years)

8. Dawson-McClure S, Miller Brotman L, Theise R, Palamar J, Kamboukos D, Barajas $\mathrm{G}$, et al. Early childhood obesity prevention in low-income, urban communities. Journal of Prevention \& Intervention in the Community 2014;42(2):152-166.

No control group

9. De Bruin E, Oort F, Bögels S, Meijer A. Efficacy of internet and group-administered cognitive behavioral therapy for insomnia in adolescents: a pilot study. Behavioral Sleep Medicine 2014;12(3):235-254.

Outside age range (12 to 19 years) 
10. Flint-Bretler T, Shochat O, Tzischinsky. The effects of a parental intervention on electronic media exposure and sleep patterns in adolescents. Sleep Medicine 2013;14(Suppl 1):e126-e127

Published abstract only, unable to locate full text

11. Lubans DR, Smith JJ, Peralta LR, Plotnikoff RC, Okely AD, Salmon J, et al. A school-based intervention incorporating smartphone technology to improve healthrelated fitness among adolescents: rationale and study protocol for the NEAT and ATLAS 2.0 cluster randomized controlled trial and dissemination study. BMJ Open 2016;6:e010448.

Outside age range (year 9 students)

12. Hinkley T, Cliff D, Okely A. Reducing electronic media use in 2-3 year-old children: feasibility and efficacy of the Family@play pilot randomized controlled trial. BMC Public Health 2015;15:779.

Sleep outcome not measured

13. Quante M, Khandpur N, Kontos E, Bakker Owens J, Redline S. "Let's talk about sleep": a qualitative examination of levers for promoting healthy sleep among sleepdeprived vulnerable adolescents. Sleep Medicine 2019;60:81-88.

Outside age range (14 to 18 years)

14. San Mauro I. The influence of healthy lifestyle habits on weight status in school aged children and adolescents. Nutrición Hospitalaria 2015;31(5):1996-2005.

Outside age range (six to 16 years); not published in English

15. Schlarb A, Brandhorst I. Mini-KiSS Online: an internet-based intervention program for parents of young children with sleep problems--influence on parental behavior and children's sleep. Nature and Science of Sleep. 2012;4.

Outside age range (six months to four years); screen time not measured

16. Andrade S, Verloigne M, Cardon G, Kolsteren P, Ochoa-Aviles A, Verstraeten R, Lachat C. School-based intervention on healthy behaviour among Ecuadorian adolescents: effect of a cluster-randomized controlled trial on screen-time. BMC Public Health 2015;15 p. n/a.

No sleep outcome measure

17. Arsenault L, Xu K, Taveras E, Hacker K. Parents' obesity-related behavior and confidence to support behavioral change in their obese child: data from the STAR study. Academic Pediatrics 2014;14(5):456-462

Not relevant, studies on the effect of a decision-making tool

18. Rito A, Carvalho M, Ramos C, Breda J. Program obesity zero (POZ) - a communitybased intervention to address overweight primary-school children from five Portuguese municipalities. Public Health Nutrition 2013;16(6):1043-51.

No control group

19. Al Khalifah R, Mok E, Legault L, Leblanc C, Constantin E. Using " $5,2,1$, 0" to promote healthy active living among school-age children attending a paediatric resident clinic: A prospective study/Utiliser le « 5, 2, 1, $0 »$ pour promouvoir une vie saine et active chez les enfants d'âge scolaire qui fréquentent. Paediatrics \& Child Health 2016;21(5):E43-E47.

No control group 
20. Novotny R, Davis J, Brutel J, Boushey C, Fialkowski M, Nigg C, et al. Effect of the children's healthy living program on young child overweight, obesity, and Acanthosis Nigricans in the US-Affiliated Pacific Region. JAMA Network Open 2018;1(16): e183896.

No description of intervention; large public health campaign over multiple site and countries, each able to modify the intervention to suit the participants, however, no details provided on the intervention variables

21. Babic MJ, Smith JJ, Morgan PJ, Lonsdale C, Plotnikoff RC, Eather N, et al. Intervention to reduce recreational screen-time in adolescents: Outcomes and mediators from the "Switch-Off 4 Healthy Minds" (S4HM) cluster randomized controlled trial. Preventive Medicine 2016;91:50-57.

Outside age range; no sleep outcome

22. Van Lippevelde W, Bere E, Verloigne M, van Stralen MM, de Bourdeaudhuij I, Lien $\mathrm{N}$. The role of family-related factors in the effects of the UP4FUN school-based family-focused intervention targeting screen time in 10-to 12-year-old children: the ENERGY project. BMC Public Health 2014;14:1471-2458.

No sleep outcome

23. Vieira J, Lima L, Silva D, Petroski E. Effectiveness of a multicomponent intervention on the screen time of Brazilian adolescents: non-randomized controlled study. Motriz: Revista de Educação Física, 2018;24(3), e0046-18.

Outside age range (10 to 16 years); no sleep outcome

24. De Leeuw R, de Bruijn M, de Weert -van Oene G, Schrijvers A. Internet and game behaviour at a secondary school and a newly developed health promotion programme: a prospective study. BMC Public Health 2010;10:544.

Outside age range (11 to 16 years); no control group, no sleep outcome

25. Busch V, De Leeuw R, Schrijvers A. Results of a Multibehavioral Health-Promoting School Pilot Intervention in a Dutch Secondary School. Journal of Adolescent Health 2013;52:400e406

Outside age range (15 to 16 years); no control group, no sleep outcome, duplicate to above

26. Dennison B, Russo T, Burdick P, Jenkins P. An Intervention to Reduce Television Viewing by Preschool Children, Archives of Pediatrics \& Adolescent Medicine 2004;158:170-6

No sleep outcome

27. Hinkley T, Cliff D, Okely A. Reducing electronic media use in 2-3 year-old children: feasibility and efficacy of the Family@play pilot randomised controlled trial. BMC Public Health 2015;15(779):1-12.

No sleep outcome

28. Robinson T, Borzekowski D. Effects of the SMART Classroom Curriculum to Reduce Child and Family Screen Time. Journal of Communication 2006;56:1-26

Sleep outcome not measured

29. De Craemer M, De Decker E, Verloigne M, De Bourdeaudhuij I, Manios Y, Cardon G. The effect of a cluster randomised control trial on objectively measured sedentary time and parental reports of time spent in sedentary activities in Belgian 
preschoolers: the ToyBox-study. International Journal of Behavioral Nutrition and Physical Activity 2016;13:1

No sleep outcome

30. Zimmerman F, Ortiz S, Christakis D, Elkun D. The value of social-cognitive theory to reducing preschool TV viewing: a pilot randomized trial. Preventive Medicine 2012;54:212-18

No sleep outcome

31. Hyeok J, Jun K, Tae C. The effect of group therapy using art program on internet/smartphone addictive behaviors of adolescents: a pilot study. Journal of the Korean Society of Biological Therapies in Psychiatry 2017;23(3):171-80

Not published in English

32. Bergh I, van Stralen M, Bjelland M, Grydeland M, Lien N, Klepp K, et al. Postintervention effects on screen behaviours and mediating effect of parental regulation: the Health in Adolescents study - a multi-component school-based randomized controlled trial. BMC Public Health 2014;14:200

No sleep outcome 


\section{Appendix VI: Outcome measures utilised by included studies}

\section{Screen related measures}

\begin{tabular}{|c|c|c|c|}
\hline Assessment tool & Study & Components & Ref/origin \\
\hline Parental report & Krossbakken $^{90}$ & $\begin{array}{l}\text { 5-point Likert scale (completely } \\
\text { agree [5] to completely disagree } \\
\text { [1] for items described in the } \\
\text { Internet Gaming Disorder DSM- } \\
5\end{array}$ & \\
\hline $\begin{array}{l}\text { Multimedia activity } \\
\text { recall for children } \\
\text { and adolescents } \\
\text { (MARCA) }\end{array}$ & Maddison $^{85}$ & $\begin{array}{l}\text { Computerised self-report, } 24 \\
\text { hours use-of-time recall of } \\
\text { physical activity and sedentary } \\
\text { behaviour }\end{array}$ & $\begin{array}{l}\text { Ridly et al. } \\
2006\end{array}$ \\
\hline \multirow[t]{6}{*}{ Self-report } & Wolfson $^{88}$ & $\begin{array}{l}\text { Self-reported screen use in the } \\
\text { hour before bed }\end{array}$ & \\
\hline & Bickham $^{86}$ & $\begin{array}{l}\text { Self-report media hours 'after } \\
\text { school yesterday' and 'last } \\
\text { Saturday' selecting from none, } \\
\text { 15min, 30min, } 1,2,3,4,5+\text { hrs }\end{array}$ & $\begin{array}{l}\text { Based on } \\
\text { Roberts, Foehr } \\
\text { \& Rideout } \\
2010\end{array}$ \\
\hline & Hammersley ${ }^{100}$ & $\begin{array}{l}\text { Self-report of screen-time hours } \\
\text { and additional questions on } \\
\text { screen rules, TV in child's } \\
\text { bedroom, frequency of viewing } \\
\text { TV during meals }\end{array}$ & $\begin{array}{l}\text { Downing et al. } \\
2015 \text { and } \\
\text { Hinkley et al. } \\
2012\end{array}$ \\
\hline & Puder $^{98}$ & $\begin{array}{l}\text { Caregiver self-report of media use } \\
\text { including } \mathrm{TV}, \text { video and } \\
\text { computer games, not details } \\
\text { given }\end{array}$ & \\
\hline & Walton $^{97}$ & $\begin{array}{l}\text { Two item questionnaire: 'On an } \\
\text { average weekday how much } \\
\text { time per day does your child } \\
\text { spend watching TV, including } \\
\text { DVDs or videos' question } \\
\text { repeated for weekend days. }\end{array}$ & $\begin{array}{l}\text { Arredondo et al. } \\
2006\end{array}$ \\
\hline & Haines $^{84}$ & $\begin{array}{l}\text { Report the number of hours the } \\
\text { child watched TV on an average } \\
\text { weekday and weekend day in } \\
\text { the past month. Presence of TV } \\
\text { in the room in which the child } \\
\text { sleeps }\end{array}$ & \\
\hline
\end{tabular}


Sleep related measures

\begin{tabular}{|c|c|c|c|}
\hline Assessment tool & Study & Components & Ref/Origin \\
\hline \multirow[t]{4}{*}{$\begin{array}{l}\text { Subset of the Child } \\
\text { Sleep Habits } \\
\text { Questionnaire } \\
\text { (CSHQ) }\end{array}$} & $\begin{array}{l}\text { Garrison \& } \\
\text { Christakis }^{62}\end{array}$ & $\begin{array}{l}\text { Frequency of 'sleep-onset } \\
\text { latency, night wakings, } \\
\text { nightmares, difficulty waking } \\
\text { in the morning and daytime } \\
\text { tiredness' with participant } \\
\text { carergiver selecting from } \\
\text { options: usually (5-7days/wk; } \\
\text { sometimes (2-4days/wk or } \\
\text { rarely (0-1 days/wk) }\end{array}$ & $\begin{array}{l}\text { Goodlin-Jones } \\
\text { et al. } 2008 \\
\text { and Owens et } \\
\text { al. } 2000\end{array}$ \\
\hline & Krossbakken $^{90}$ & $\begin{array}{l}\text { Used 'bedtime resistance' } \\
\text { subscale and additional } \\
\text { question for parents to } \\
\text { determine if the behaviour was } \\
\text { problematic or not }\end{array}$ & \\
\hline & Hammersley ${ }^{100}$ & $\begin{array}{l}\text { Used } 4 \text { questions assessing sleep } \\
\text { latency, sleep reluctance, } \\
\text { difficulty sleeping and } \\
\text { difficulty falling asleep in own } \\
\text { bed }\end{array}$ & \\
\hline & Hammersley ${ }^{100}$ & $\begin{array}{l}\text { Actigraph GT3X+accelerometer } \\
\text { for measuring sleep based on } \\
\text { wearing it for a minimum of } 3 \\
\text { nights }\end{array}$ & \\
\hline $\begin{array}{l}\text { Adolescent Health } \\
\text { and Sleep Efficacy } \\
\text { Scale }\end{array}$ & Wolfson $^{88}$ & $\begin{array}{l}\text { Participants rated how sure they } \\
\text { were that they could handle } \\
\text { each activity (six sleep hygiene } \\
\text { practices) }\end{array}$ & $\begin{array}{l}\text { Rossi et al. } \\
2002\end{array}$ \\
\hline $\begin{array}{l}\text { Adolescent Sleep } \\
\text { Hygiene Scale }\end{array}$ & Wolfson ${ }^{88}$ & $\begin{array}{l}\text { 28-items self-report on } \\
\text { frequency of sleep hygiene } \\
\text { practices }\end{array}$ & $\begin{array}{l}\text { Lebourgeois et } \\
\text { al. } 2005\end{array}$ \\
\hline \multirow[t]{2}{*}{$\begin{array}{l}\text { Adapted School } \\
\text { Sleep Habits } \\
\text { Questionnaire }\end{array}$} & Wolfson $^{88}$ & $\begin{array}{l}\text { Self-report of 'usual sleep and } \\
\text { wake behaviours over the past } \\
2 \text { weeks' }\end{array}$ & $\begin{array}{l}\text { Wolfson \& } \\
\text { Carskadon } \\
1998\end{array}$ \\
\hline & $\begin{array}{r}\text { Tamura \& } \\
\text { Tanaka }^{87}\end{array}$ & $\begin{array}{l}\text { Self-report questionnaire of } 10 \\
\text { daily sleep-related lifestyle } \\
\text { habits, selecting 'practice', 'do } \\
\text { not practice' or 'can practice', } \\
\text { including: 'refraining from } \\
\text { watching television or videos } \\
\text { before sleep' and 'refraining }\end{array}$ & $\begin{array}{l}\text { Tanaka, } \\
\text { Furutani } \\
2012 ; \\
\text { Uchiyama } \\
2002\end{array}$ \\
\hline
\end{tabular}


from playing videogames

before sleep'

Tamura \&

Tanaka $^{87}$

Self-report of sleep and daytime functioning scale of 12 items

Sleep diary

Tamura \&

Tanaka $^{87}$

Self-report of bedtime, light-off time, wake-up time, mood at awakening, sleepiness during class

\section{Multimedia Activity \\ Recall for Children and Adolescents}

(MARCA)

Self-report
Maddison $^{85}$

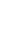

Bickham $^{86}$

Walton $^{97}$
Haines $^{84}$

\section{Puder $^{98}$}

Haines
Computerised self-report, 24 hours use-of-time recall of physical activity and sedentary behaviour

Child self-report of "what time usually go to bed, amount of time it takes to go to sleep and time usually wake up'

Ridly et al. 2006

Based on Monk et al. 2003;

Wolfson

2003
Caregiver self-report of child's sleep duration; no details given

2-item questionnaire for caregiver: 'what time does your child usually wake up/go to bed on weekdays

Parent to report the average amount of daily sleep, including naps, and a separate question on bedtime and waketimes in the past month 


\section{Appendix VII: Statement of authorship}

\section{a. Protocol}

\section{Statement of Authorship}

\begin{tabular}{|c|c|}
\hline Title of Paper & $\begin{array}{l}\text { Effeclivenass of interventlions to control screen use and children's sleep, cognitive and behavioural outcomes: } \\
\text { a systemalic review protocol }\end{array}$ \\
\hline Publication Status & $\begin{array}{l}\Gamma \text { Accepted for Publication } \\
\Gamma \text { Unpublished and Unsubmilted w ork w ritten in } \\
\text { manuscript style }\end{array}$ \\
\hline Publication Details & $\begin{array}{l}\text { Martin K, Porritt K, Aromataris E. Effectiveness of interventions to control screen use and } \\
\text { children's sleep, cognilive and behavioral outcomes: a systematic review protocol. } \\
\text { JBI Database of System Rev Implement Rep. 2018;16(6):1338-1345. }\end{array}$ \\
\hline
\end{tabular}

\section{Principal Author}

\begin{tabular}{|c|c|c|}
\hline Name of Principal Author (Candidate) & \multicolumn{2}{|l|}{ Katie Martin } \\
\hline Contribution to the Paper & \multicolumn{2}{|c|}{ Conceptualised topic, developed PICO question, wrote manuscript and acted as corresponding author } \\
\hline Overall percentage $(\%)$ & \multicolumn{2}{|l|}{$90 \%$} \\
\hline Certification: & \multicolumn{2}{|c|}{$\begin{array}{l}\text { This paper reports on original research I conducted during the period of my Higher Degree by } \\
\text { Research candidature and is not subject to any obligations or contractual agreements with a } \\
\text { third party that would constrain its inclusion in this thesis. I am the primary author of this paper. }\end{array}$} \\
\hline Signature & Date & $13 / 11 / 2020$ \\
\hline
\end{tabular}

\section{Co-Author Contributions}

By signing the Statement of Authorship, each author certifies that:

i. the candidate's stated contribution to the publication is accurate (as detailed above);

ii. permission is granted for the candidate in include the publication in the thesis; and

iii. the sum of all co-author contributions is equal to $100 \%$ less the candidate's stated contribution.

\begin{tabular}{|l|l|l|l|l|l|}
\hline Name of Co-Author & Kylie Porritt \\
\hline Contribution to the Paper & Assisted in manuscript evaluation \\
& & & & \\
& & & Date & $23 / 11$ & 2020 \\
\hline
\end{tabular}

\begin{tabular}{|l|l|l|}
\hline Name of Co-Author & Edoardo Aromataris \\
\hline Contribution to the Paper & Supervised development of the work and manuscript evaluaiton \\
\hline Signature & & \\
\hline
\end{tabular}

Please cut and paste additional co-author paness nere as required. 


\section{b. Systematic review}

\section{Statement of Authorship}

\begin{tabular}{|l|l|}
\hline Title of Paper & $\begin{array}{l}\text { Interventions to control children's screen-use and their effect on sleep: } \\
\text { a systematic review and meta-analysis. }\end{array}$ \\
\hline Publication Status & $\sqrt{ }$ : Published \\
& $\Gamma$ Submitted for Publication $\quad \begin{array}{l}\text { Anpublished and Unsubmitted w ork w ritten in } \\
\text { manuscript style }\end{array}$ \\
\hline Publication Details & $\begin{array}{l}\text { Martin K, Bednarz J, Aromataris E. Interventions to control chilcren's screen-use } \\
\text { and their effect on sleep: a systematic review and meta-analysis. J Sleep Res. 2020;00:e13130. }\end{array}$ \\
\hline
\end{tabular}

\section{Principal Author}

\begin{tabular}{|c|c|c|}
\hline Name of Principal Author (Candidate) & \multicolumn{2}{|l|}{ Katie Martin } \\
\hline Contribution to the Paper & \multicolumn{2}{|c|}{$\begin{array}{l}\text { Performed systematic review search, elegibility screening, critical appraisal, statistical calculations, } \\
\text { meta-analysis and data synthesis. Wrote manusctipt and acted as corresponding author. }\end{array}$} \\
\hline Overall percentage $(\%)$ & \multicolumn{2}{|l|}{$85 \%$} \\
\hline Certification: & \multicolumn{2}{|c|}{$\begin{array}{l}\text { This paper reports on original research I conducted during the period of my Higher Degree by } \\
\text { Research candidature and is not subject to any obligations or contractual agreements with a } \\
\text { third party that would constrain its inclusion in this thesis. I am the primary author of this paper. }\end{array}$} \\
\hline Signature & Date & $13 / 11 / 2020$ \\
\hline
\end{tabular}

\section{Co-Author Contributions}

By signing the Statement of Authorship, each author certifies that:

i. the candidate's stated contribution to the publication is accurate (as detailed above);

ii. permission is granted for the candidate in include the publication in the thesis; and

iii. the sum of all co-author contributions is equal to $100 \%$ less the candidate's stated contribution.

\begin{tabular}{|l|l|l|l|}
\hline Name of Co-Author & Jana Bednarz \\
\hline Contribution to the Paper & $\begin{array}{l}\text { Assisted in selection of statistical processes and supervision of statistical calculations in } \\
\text { data analysis. Assisted in manusctipt evaluation. }\end{array}$ \\
\cline { 1 - 1 } Signature & & Date & $13 / 11 / 2020$ \\
\hline
\end{tabular}

\begin{tabular}{|l|l|l|l|}
\hline Name of Co-Author & Edoardo Aromataris \\
\hline Contribution to the Paper & Supervised development of the work, assisted in data interpretation and manuscript evaluaiton. \\
\hline Signature & & Date & $13 / 11 / 2020$ \\
\hline
\end{tabular}

Please cut and paste additional co-author panels here as required. 


\section{References}

1. Rideout V. Measuring time spent with media: the Common Sense census of media use by US 8- to 18-year-olds. J Child Media 2016;10(1):138-144.

2. Rhodes A. Australian Child Health Poll. Poll 7, Screen time and kids: What's happening in our homes? [internet]. The Royal Children's Hospital, Melbourne. 2017 [cited 2020 Jun 01]. Available from: http://www.rchpoll.org.au/wpcontent/uploads/2017/06/ACHP-Poll7_Detailed-Report-June21.pdf.

3. LeBourgeois M, Hale L, Chang A, Akacem L, Montgomery-Downs H, Buxton O. Digital media and sleep in childhood and adolescence. Pediatrics 2017;140(Suppl 2):S92-S96.

4. Australian Bureau of Statistics. Australian Health Survey: physical activity, 20112012. [internet]. 2013 [cited 2017 Aug 05]. Available from https://www.abs.gov.au/statistics/health-survey-physical-activity/latest-release

5. Braithwaite I, Stewart AW, Hancox RJ, Beasley R, Murphy R, Mitchel E. The Worldwide Association between Television Viewing and Obesity in Children and Adolescents: Cross Sectional Study. PLoS ONE 2013;8(9):e74263.

6. Hale L, Guan S. Screen time and sleep among school-aged children and adolescents: a systematic literature review. Sleep Med Rev 2015 June;21:50-8.

7. Paavonen EJ, Pennonen M, Roine M, Valkonen S, Lahikainen AR. TV exposure associated with sleep disturbances in 5- to 6-year-old children. J Sleep Res 2006;15(2):154-61.

8. Ivarsson M, Adnerson M, Åkerstedt T, Lindbald F. The effect of violent and nonviolent video games on heart rate variability, sleep, and emotions in adolescents with different violent gaming habits. Psychosom Med 2013;75(4);390-6.

9. Hill D, Ameenuddin N, Reid Chassiakos Y, Cross C, Hutchinson J, Levine A, et al. Media and young minds. Pediatrics 2016;138(5):e20162591.

10. Friedrich RR, Polet JP, Schuch I, Wagner MB. Effect of intervention programs in schools to reduce screen time: a meta-analysis. J Pediatr (Rio J) 2014;90(3):23241.

11. Maniccia DM, Davison KK, Marshall SJ, Manganello JA, Dennison BA. A metaanalysis of interventions that target children's screen time for reduction. Pediatrics 2011;128(1):e193-210.

12. Marsh S, Foley LS, Wilks DC, Maddison R. Family-based interventions for reducing sedentary time in youth: a systematic review of randomized controlled trials. Obes Rev 2014;15(2):117-33.

13. Ramsey Buchanan L, Rooks-Peck CR, Finnie RK, Wethington HR, Jacob V, Fulton JE, et al. Reducing recreational sedentary screen time: a community guide systematic review. Am J Prev Med 2016;50(3):402-15.

14. Schmidt ME, Haines J, O'Brien A, McDonald J, Price S, Sherry B, et al. Systematic review of effective strategies for reducing screen time among young children. Obesity 2012;20(7):1338-54.

15. Steeves J, Thompson D, Bassett D, Fitzhugh E, Raynor H. A review of different behavior modification strategies designed to reduce sedentary screen behaviors in children. J Obes 2011;2012:379215. 
16. Wahi G, Parkin PC, Beyene J, Uleryk EM, Birken CS. Effectiveness of interventions aimed at reducing screen time in children: a systematic review and meta-analysis of randomized controlled trials. Arch Pediatr Adolesc Med 2011;165(11):979-86.

17. Wu L, Sun S, He Y, Jiang B. The effect of interventions targeting screen time reduction: A systematic review and meta-analysis. Medicine 2016; 95(27):e4029.

18. Martin K, Porritt K, Aromataris E. Effectiveness of interventions to control screen use and children's sleep, cognitive and behavioral outcomes: a systematic review protocol. JBI Database System Rev Implement Rep 2018;16(6):1338-45.

19. Martin K, Bednarz J, Aromataris E. Interventions to control children's screen-use and their effect on sleep: a systematic review and meta-analysis. J Sleep Res 2020;00:e13130.

20. Halal CS, Nunes ML. Education in children's sleep hygiene: which approaches are effective? A systematic review. J Pediatr (Rio J) 2014;90(5):449-56.

21. Sadeh A, Mindell JA, Luedtke K, Wiegand B. Sleep and sleep ecology in the first 3 years: a web-based study. J Sleep Res 2009;18(1):60-73.

22. Yu M, Baxter J. LSAC Annual Statistical Report 2015. Chapter 5, Australian children's screen time and participation in extracurricular activities. [internet]. [cited 2020 Mar 02]. Available from https://growingupinaustralia.gov.au/researchfindings/annual-statistical-report-2015/australian-childrens-screen-time-andparticipation-extracurricular

23. Wethington H, Pan L, Sherry B. The association of screen time, television in the bedroom, and obesity among school-aged youth: 2007 National Survey of Children's Health. J Sch Health 2013;83(8):573-81.

24. Ye S, Chen L, Wang Q, Li Q. Correlates of screen time among 8-19-year-old students in China. BMC Public Health. 2018;18:467.

25. Dennison BA, Russo TJ, Burdick PA, Jenkins PL. An intervention to reduce television viewing by preschool children. Arch Pediatr Adolesc Med 2004;158(2):170-6.

26. Mireku MO, Barker MM, Mutz J, Dumontheil I, Thomas MS, Röösli M, et al. Night-time screen-based media device use and adolescents' sleep and healthrelated quality of life. Environ Int 2019;124:66-78.

27. Foulds HJ, Rodgers CD, Duncan V, Ferguson LJ. A systematic review and metaanalysis of screen time behaviour among North American indigenous populations. Obes Rev 2016;17(5):455-66.

28. Barber S, Kelly B, Wright J. Prevalence, trajectories and determinants of television viewing time in an ethnically diverse sample of young children from the UK. Int J Behav 2017;14:88.

29. Yang-Huang J, van Grieken A, Wang A, Jaddoe V, Jansen W, Raat H. Ethnic background and childrens television viewing trajectories, the generation R Study. PLoSONE 2018;13(12):e0209375.

30. Goncalves WS, Byrne R, Viana M, Trost S. Parental influences on screen time and weight status among preschool children from Brazil: a cross-sectional study. Int J Behav N 2019;16(1):27.

31. Emond JA, Tantum LK, Gilbert-Diamond D, Kim SJ, Lansigan RK, Neelon SB. Household chaos and screen media use among preschool-aged children: a crosssectional study. BMC Public Health 2018;18(1):1210. 
32. Bank A, Barr R, Calvert S, Parrott W, McDonough S, Rosenblum K. Maternal depression and family media use: a questionnaire and diary analysis. J Child Fam Stud 2012;21(2):208-16.

33. Beyens I, Eggermont S, Nathanson A. Understanding the relationship between mothers' attitudes toward television and children's television exposure: a longitudinal study of reciprocal patterns and the moderating role of maternal stress. Media Psychol 2016;19(4):638-65.

34. Carson V, Hunter S, Kuzik N, Gray CE, Poitras VJ, Chaput JP, et al. Systematic review of sedentary behaviour and health indicators in school-aged children and youth: an update. Appl Physiol Nutr Metab 2016;41(6 Suppl 3):S240-S265.

35. Biddle SJ, García Bengoechea E, Wiesner G. Sedentary behaviour and adiposity in youth: a systematic review of reviews and analysis of causality. Int J Behav Nutr Phys Act 2017;14(1):43.

36. Television and growing up: the impact of televised violence. Report to the Surgeon General United States Public Health Service. Washington: United States National Institute of Mental Health 1972;76-100

37. Zhang G, Wu L, Zhou L, Lu W, Mao C. Television watching and risk of childhood obesity: a meta-analysis. Eur J Public Health 2016;26(1):13-18.

38. Guo C, Zhou Q, Zhang D, Qin P, Li Q, Tian G, et al. Association of total sedentary behaviour and television viewing with risk of overweight/obesity, type 2 diabetes and hypertension: a dose-response meta-analysis. Diabetes Obes Metab 2020;22(1):79-90.

39. Boulos R, Kuross Vikre E, Oppenheimer S, Chang H, Kanarek R. ObesiTV: How television is influencing the obesity epidemic. Physiol Behav 2012;107(1):146-53.

40. Coker TR, Elliott MN, Schwebel DC, Windle M, Toomey SL, Tortolero SR, et al. Media violence exposure and physical aggression in fifth-grade children. Acad Pediatr 2015;15(1):82-8.

41. Otto MW, Henin A, Hirshfeld-Becker DR, Pollack MH, Biederman J, Rosenbaum JF. Posttraumatic stress disorder symptoms following media exposure to tragic events: Impact of 9/11 on children at risk for anxiety disorders. J Anxiety Disord 2007;21(7):888-902.

42. Allen MS, Vella SA. Screen-based sedentary behaviour and psychosocial wellbeing in childhood: cross-sectional and longitudinal associations. Ment Health Phys Act 2015;9:41-7.

43. Twenge J, Campbell K. Associations between screen time and lower psychological well-being among children and adolescents: Evidence from a population-based study. Prev Med Rep 2018;12:271-83.

44. Kostyrka-Allchorne K, Cooper NR, Simpson A. The relationship between television exposure and children's cognition and behaviour: a systematic review. Dev Rev 2017;44:19-58.

45. Babic MJ, Smith JJ, Morgan PJ, Eather N, Plotnikoff RC, Lubans DR. Longitudinal associations between changes in screen-time and mental health outcomes in adolescents. Ment Health Phys Act 2017;12:124-31.

46. Howie EK, Joosten J, Harris CJ, Straker L. Associations between meeting sleep, physical activity or screen time behaviour guidelines and academic performance in Australian school children. BMC Public Health 2020;20:520.

47. Adelantado-Renau M, Moliner-Urdiales D, Cavero-Redondo I. Association between screen media use and academic performance among children and 
adolescents: a systematic review and meta-analysis. JANA Pediatr 2019;173(11):1058-67.

48. Dube N, Khan K, Loehr S, Chu Y, Veugelers P. The use of entertainment and communication technologies before sleep could affect sleep and weight status: a population-based study among children. Int J Behav Nutri 2017;14:97.

49. Carter B, Rees P, Hale L, Bhattacharjee D, Paradkar M. Association between portable screen-based media device access or use and sleep outcomes: a systematic review and meta-analysis. JAMA Pediatr 2016;170(12):1202-8.

50. Michels N, De Henauw S, Eiben G, Hadjigeorgiou C, Hence S, Hunsberger M, et al. Effect of the IDEFICS multilevel obesity prevention on children's sleep duration. Obes Rev 2015;16(suppl 2):68-77.

51. Garrison M, Liekweg B, Christakis D. Media use and child sleep: the impact of content, timing, and environment. Pediatrics 2011;128(1):29-35.

52. Peracchia S, Giuseppe C. Exposure to video games: effects on sleep and on postsleep cognitive abilities. A systematic review of experimental evidence. Sleep Sci 2018;11(4):302-14.

53. Chindamo S, Buja A, DeBattisti E, Terraneo A, Marini E, Gomez Perez LJ, et al. Sleep and new media usage in toddlers. Eur J Pediatr 2019;178(4):483-90.

54. Parent J, Sanders W, Forehand R. Youth screen time and behavioral health problems: the role of sleep duration and disturbances. J Dev Behav Pediatr 2016;37(4):277-84.

55. Rapoport D, Milanaik R, Muthiah N, Adesman A. Early childhood digital media use: inverse associations with sleep time consistency and sleep duration. Pediatrics 2019;144(2 Meeting Abstract):52.

56. Lange K, Cohrs S, Skarupke C, Görke M, Szagun B, Schlack R. Electronic media use and insomnia complaints in German adolescents: gender differences in use patterns and sleep problems. J Neural Transm (Vienna) 2017;124(Suppl 1):79-87.

57. Akacem L. Understanding the circadian physiology in early childhood: the role of napping and light at night. Dissertation Abstracts International: Section B: The Sciences and Engineering. 2017;78(3-B(E)).

58. Kubota T, Uchiyama M, Suzuki H, Shibui K, Kim K, Tan X, et al. Effects of nocturnal bright light on saliva melatonin, core body temperature and sleep propensity rhythms in human subjects. Neurosci Res 2002;42(2):115-22.

59. Chang A, Aeshbach D, Duffy J, Czeisler C. Evening use of light emitting E-readers negatively affects sleep, circadian timing and next-morning alertness. Proceedings of the National Academy of Sciences 2015;112(4):1232-7.

60. Rångtell FH, Ekstrand E, Rapp L, Lagermalm A, Liethof L, Búcaro M, et al. Two hours of evening reading on a self-luminous tablet vs. reading a physical book does not alter sleep after daytime bright light exposure. Sleep Med 2016;23:111-8.

61. Šmotek M, Fárková E, Manková D, Kopřivová J. Evening and night exposure to screens of media devices and its association with subjectively perceived sleep: Should "light hygiene" be given more attention? Sleep Health 2020;6(4):498-505.

62. Garrison MM, Christakis DA. The Impact of a healthy media use intervention on sleep in preschool children. Pediatrics. 2012;130(3):492-9.

63. Hiscock H, Canterford L, Ukoumunne OC, Wake M. Adverse associations of sleep problems in Australian preschoolers: national population study. Pediatrics 2007;119(1):86-93. 
64. Cespedes EM, Rifas-Shiman SL, Redline S, Gillman MW, Peña M-M, Taveras EM. Longitudinal associations of sleep curtailment with metabolic risk in midchildhood. Obesity 2014;22(12):2586-92.

65. Chaput J, Gray C, Poitras V, Carson V, Gruber R, Birken C, et al. Systematic review of the relationships between sleep duration and health indicators in the early years (0-4 years). BMC Public Health 2017;17(Suppl 5):855.

66. Meldrum RC, Restivo E. The behavioural and health consequences of sleep deprivation among U.S. high school students: relative deprivation matters. Prev Med 2014;63:24-8.

67. Cedernaes J, Schiöth HB, Benedict C. Determinants of shortened, disrupted or mistimed sleep and associated metabolic health consequences in healthy humans. Diabetes 2015;64(4):1073-80.

68. Hill D, Ameenuddin N, Reid Chassiakos Y, Cross C, Hutchinson J, Levine A, et $a l$. Media and Young Minds. Pediatrics 2016;138(5).

69. Children, Adolescents, and Television. Pediatrics 2001;107(2):423-6.

70. Houghton S, Hunter SC, Rosenberg M, Wood L, Zadow C, Martin K, et al. Virtually impossible: limiting Australian children and adolescents daily screen based media use. BMC Public Health 2015;15(1):5.

71. World Health Organisation. Guidelines on physical activity, sedentary behaviour and sleep for children under 5 years of age. [internet]. 2019 [cited 2020 Mar 01]. Available from: https://www.who.int/publications/i/item/9789241550536.

72. Hale L, Kirschen G, LeBourgeois M, Gradisar M, Garrison M, MontgomeryDowns $\mathrm{H}$, et al. Youth screen media habits and sleep, sleep-friendly screen behaviour recommendations for clinicians, educators and parents. Child Adolesc Psychiatric Clin N Am 2018;27:220-45.

73. Calhoun S, Fernandez-Mendoza J, Vgontzas A, Liao D, Bixler E. Prevalence of insomnia symptoms in a general population sample of young children and preadolescents: gender effects. Sleep Med 2014;15(1):91-5.

74. Gruber R, Cassoff J, Knäuper B. Sleep hygiene education in pediatric community settings: rationale and practical suggestions for incorporating healthy sleep education into pediatric practice. Pediatr Clin North Am 2011;58(3):735-54.

75. Bowers J, Moyer A. Adolescent sleep and technology-use rules: results from the California Health Interview Survey. Sleep Health 2020;6(1):19-22.

76. Altenburg TM, Kist-van Holthe J, Chinapaw MJM. Effectiveness of intervention strategies exclusively targeting reductions in children's sedentary time: a systematic review of the literature. Int J Behav. 2016;13(1):65.

77. Comer J, Furr J, Beidas R, Weiner C, Kendall P. Children and terrorism-related news: training parents in coping and media literacy. J Consult Clin Psychol 2008;76(4):568-78.

78. Bickham D, Slaby R. Effects of a media literacy program in the US on children's critical evaluation of unhealthy media messages about violence, smoking and food. J Children Media 2012;6(2):255-72.

79. Fingar K, Jolls T. Evaluation of a school-based violence prevention media literacy curriculum. Injury Prev 2014;20(3):183-90.

80. Tufanaru C, Munn Z, Aromataris E, Campbell J, Hopp L. Chapter 3: Systematic reviews of effectiveness. In: Aromataris E, Munn Z (Editors). JBI Manual for 
Evidence Synthesis. JBI, 2020. [cited 2020 Oct 19]. Available from https://synthesismanual.jbi.global.

81. Biddle SJ, Petrolini I, Pearson N. Interventions designed to reduce sedentary behaviours in young people: a review of reviews. Br J Sports Med 2014;48(3):182186.

82. van Grieken A, Ezendam NP, Paulis WD, van der Wouden JC, Raat H. Primary prevention of overweight in children and adolescents: a meta-analysis of the effectiveness of interventions aiming to decrease sedentary behaviour. Int J Behav 2012;9(1):61.

83. Tan E, Healey D, Gray A, Galland BC. Sleep hygiene intervention for youth aged 10 to 18 years with problematic sleep: a before-after pilot study. BMC Pediatr. 2012;12(1):189.

84. Haines J, McDonald J, O'Brien A, Sherry B, Bottino CJ, Schmidt ME, et al. Healthy Habits, Happy Homes: randomized trial to improve household routines for obesity prevention among preschool-aged children. JAMA Pediatr 2013;167(11):1072-79.

85. Maddison R, Marsh S, Foley L, Epstein L, Olds T, Dewes O, et al. Screen-time weight loss intervention targeting children at home (SWITCH): a randomised controlled trial. BioMed Central 2014;11:111.

86. Bickham DS, Hswen Y, Slaby RG, Rich M. A preliminary evaluation of a schoolbased media education and reduction intervention. J Prim Prev 2018;39(3):229-45.

87. Tamura N, Tanaka H. Effects of sleep education with self-help treatment for elementary schoolchild with nocturnal lifestyle and irritability. Sleep Biol Rhythms 2014;12:169-79.

88. Wolfson AR, Harkins E, Johnson M, Marco C. Effects of the young adolescent sleep smart program on sleep hygiene practices, sleep health efficacy, and behavioral well-being. Sleep Health 2015;1(3):197-204.

89. Meltzer LJ, Mindell JA. Systematic review and meta-analysis of behavioral interventions for pediatric insomnia. J Pediatr Psychol 2014;39(8):932-48.

90. Krossbakken E, Torsheim T, Mentzoni R, King D, Bjorvatn B, Lorvic I, et al. The effectiveness of a parental guide for prevention of problematic video gaming in children: a public health randomized controlled intervention study. J Behav 2018;7(1):52-61.

91. Paulus FW, Ohmann S, von Gontard A, Popow C. Internet gaming disorder in children and adolescents: a systematic review. Dev Med Child Neurol 2018;60:645-59.

92. $\mathrm{Li} \mathrm{A}, \mathrm{Lo} \mathrm{B}$, Cheng $\mathrm{C}$. It is the family context that matters: concurrent and predictive effects of aspects of parent-child interaction on video gaming-related problems. Cyberpsychol Behav Soc Netw 2018;21(6):374-80.

93. Bonnaire C, Liddle H, Har A, Nielson P, Phan O. Why and how to include parents in the treatment of adolescents presenting internet gaming disorder? J Behav 2019;8(2):201-12.

94. Yoong SL, Chai LK, Williams CM, Wiggers J, Finch M, Wolfenden L. Systematic review and meta-analysis of interventions targeting sleep and their impact on child body mass index, diet, and physical activity. Obesity 2016;24(5):1140-7.

95. Blake M, Sheeber L, Youssef G, Raniti M, Allen N. Systematic review and metaanalysis of adolescent cognitive-behavioral sleep interventions. Clin Child Fam Psychol Rev 2017; 20(3):227-49. 
96. Jago R, Sebire S, Lucas P, Turner K, Bentley G, Goodrend J, et al. Parental modelling, media equipment and screen-viewing among children: cross-sectional study. BMJ Open. 2013;3:e002593.

97. Walton K, Filion J, Gross D, Morrongiello B, Darlington G, Simpson J, et al. Parents and tots together: Pilot randomized controlled trial of a family based obesity prevention intervention in Canada. Can J Public Health 2015;106(8):e555e562.

98. Puder J, Marques-Vidal P, Schindler C, Zahner L, Niederer I, Burgi F, et al. Effect of a multidimensional lifestyle intervention on fitness and adiposity in predominantly migrant preschool children (Ballabeina): cluster randomised controlled trial. BMJ 2011;343.

99. Masur EF, Flynn V, Olson J. The presence of background television during young children's play in American homes. J Children Media 2015;9(3):349-67.

100. Hammersley M, Okely A, Batterham M, Jones R. An internet-based childhood obesity prevention program (Time2bHealthy) for parents of preschool-aged children: randomized controlled trial. J Med Internet Res 2019;21(2):e11964.

101. Tomayko E, Prince R, Cronin K, Kim K, Parker T, Adams A. The healthy children, strong families 2 (HCSF2) randomized controlled trial improved healthy behaviors in American Indian families with young children. Curr Dev Nutr 2019;3(Suppl 2):53-62.

102. Kaptsis D, King DL, Delfabbro PH, Gradisar M. Withdrawal symptoms in internet gaming disorder: A systematic review. Clin Psychol Rev 2016;43:58-66.

103. Gower A, Moreno M. A novel approach to evaluating mobile smartphone screen time for iPhones: feasibility and preliminary findings. JMIR Mhealth Uhealth 2018;6(11):e11012.

104. Xiao Q, Chaput J, Olds T, Fogelholm, M, Hu G, Lambert EV, et al. Sleep characteristics and health-related quality of life in 9- to 11-year-old children from 12 countries. Sleep Health 2020;6(1):4-14.

105. Ong A, Gillespie B. Overview of smartphone applications for sleep analysis. World J Otorhinolaryngol Head Neck Surg 2016;2(1):45-9. 